\title{
THE CRITICAL GROUP OF A LINE GRAPH
}

\author{
ANDREW BERGET, ANDREW MANION, MOLLY MAXWELL, AARON POTECHIN, \\ AND VICTOR REINER
}

\begin{abstract}
The critical group of a graph is a finite abelian group whose order is the number of spanning forests of the graph. This paper provides three basic structural results on the critical group of a line graph.

- The first deals with connected graphs containing no cut-edge. Here the number of independent cycles in the graph, which is known to bound the number of generators for the critical group of the graph, is shown also to bound the number of generators for the critical group of its line graph.

- The second gives, for each prime $p$, a constraint on the $p$-primary structure of the critical group, based on the largest power of $p$ dividing all sums of degrees of two adjacent vertices.

- The third deals with connected graphs whose line graph is regular. Here known results relating the number of spanning trees of the graph and of its line graph are sharpened to exact sequences which relate their critical groups.

The first two results interact extremely well with the third. For example, they imply that in a regular nonbipartite graph, the critical group of the graph and that of its line graph determine each other uniquely in a simple fashion.
\end{abstract}

\section{Contents}

1. Introduction and main results

1.1. The hypothesis of no cut-edge

1.2. The hypothesis that degree sums of adjacent vertices are divisible by $p$

1.3. The regularity hypothesis

2. Some theory of lattices

2.1. Rational orthogonal decompositions

2.2. Functoriality

2.3. Pontrjagin duality

3. The critical group of a graph

3.1. Cycles, bonds, Laplacians, and spanning trees 8

3.2. A presentation for $K($ line $G) \quad 10$

$\begin{array}{lll}\text { 3.3. Lorenzini's result on edge subdivisions } & 10\end{array}$

3.4. A non-standard treatment of the complete graph 11

4. Proof of Theorem $1.1 \quad 12$

5. Proof of Theorem $1.2 \quad 15$

6. Proof of Theorem $1.3 \quad 18$

6.1. Defining the morphism $f \quad 18$

6.2. The kernel and cokernel of $f$ are $d$-torsion 20

Key words and phrases. Critical group, line graph, regular graph.

Work of all authors supported by NSF grants DMS-0245379 and DMS-0601010, and completed partly during REU programs at the University of Minnesota during the summers of 2003, 2004 and 2008. 
6.3. Analyzing the cokernel 21

6.4. Analyzing the kernel 22

7. Proof of Corollary 1.4 25

8. Proof of Theorem 1.5 27

\begin{tabular}{ll} 
8.1. & Defining the morphism $g$ \\
\hline
\end{tabular}

8.2. Analyzing its kernel and cokernel $\quad 29$

9. Examples $\quad 31$

9.1. The complete graph $K_{n} \quad 31$

9.2. The complete bipartite graph $K_{n_{1}, n_{2}}$

9.3. The $d$-dimensional cube $\quad 32$

9.4. The Platonic solids $\quad 33$

References

\section{INTRODUCTION AND MAIN RESULTS}

The critical group $K(G)$ of a graph $G$ is a finite abelian group whose order is the number $\kappa(G)$ of spanning forests of the graph. One can define $K(G)$ in several ways, closely related to the cycle and bond spaces of the graph, the graph Laplacian, as well as a certain chip-firing game that is played on the vertices of the graph and is called the abelian sandpile model in the physics literature. The interested reader can find some of the standard results on $K(G)$ in [1, 4] and [8, Chapter 13]. Some of this material is reviewed in Sections 2 and 3 below, along with unpublished results ${ }^{1}$ from the bachelor's thesis of D. Treumann [18] on functoriality for critical groups.

The critical group $K(G)$ and its relation to the structure of the graph $G$ remain, in general, mysterious. The goal of this paper is to compare the structure of the critical group of a simple graph (that is, a graph having no multiple edges and no loops) with that of the critical group of its line graph. Recall that for a graph $G=(V, E)$, its line graph line $G=\left(V_{\text {line } G}, E_{\text {line } G}\right)$ has vertex set $V_{\text {line } G}:=E$, the edge set of $G$, and an edge in $E_{\text {line } G}$ corresponding to each pair of edges in $E$ that are incident at a vertex. Our main
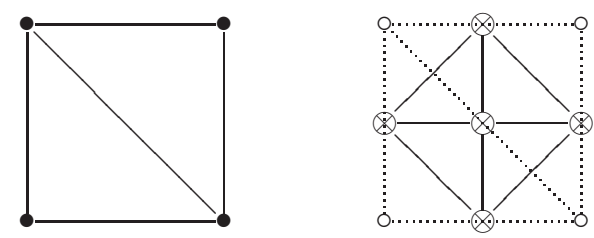

Figure 1. A graph $G$ and its line graph line $G$ with $G$ underlayed.

results say that, under three different kinds of hypotheses, the structure of $K$ (line $G$ ) is not much more complicated than that of $K(G)$, as we now explain.

1.1. The hypothesis of no cut-edge. It is well-known, and follows from one of the definitions of $K(G)$ in Section 3, that the number $\beta(G)$ of independent cycles in $G$ gives an

\footnotetext{
${ }^{1}$ The authors thank David Treumann for allowing them to include here some of his results.
} 
upper bound on the number of generators required for $K(G)$; that is,

$$
K(G)=\bigoplus_{i=1}^{\beta(G)} \mathbb{Z}_{d_{i}}
$$

where $\mathbb{Z}_{d}$ denotes the cyclic group $\mathbb{Z} / d \mathbb{Z}$ (not the $d$-adic integers), and the $d_{i}$ are positive integers (some of which may be 1). Our first main result asserts that the same bound on the number of generators holds for $K$ (line $G$ ) when one assumes that $G$ is 2-edge-connected, that is, $G$ is connected and contains no cut-edge.

Theorem 1.1. When the simple graph $G$ is 2-edge-connected, the critical group $K$ (line $G$ ) can be generated by $\beta(G)$ elements.

Note that one needs some hypothesis on the graph $G$ for this conclusion to hold. For example, a star graph $K_{1, n}$ (= one vertex of degree $n$ connected to $n$ vertices of degree one) has $\beta\left(K_{1, n}\right)=0$. However, its line graph is the complete graph $K_{n}$ and thus, according to Proposition 3.2 below, has critical group $K$ (line $\left.K_{1, n}\right)=\mathbb{Z}_{n}^{n-2}$, requiring $n-2$ generators. Theorem 1.1 is proven in Section 4, using a useful presentation of $K($ line $G$ ) given in Section 3.2.

1.2. The hypothesis that degree sums of adjacent vertices are divisible by $p$. As $K($ line $G)$ is a finite abelian group, its structure is completely determined once one knows, for each prime $p$, the structure of its $p$-primary component or $p$-Sylow subgroup $\operatorname{Syl}_{p}(K($ line $G)$ ). Section 5 below proves the following stringent constraint on this $p$-primary structure, based on the largest power $k(p)$ such that $p^{k(p)}$ divides all of the $\operatorname{sums} \operatorname{deg}_{G}(v)+\operatorname{deg}_{G}(w)$ as one runs through all edges $e=\{v, w\}$ in the edge set $E$ of $G$. Here $\operatorname{deg}_{G}(v)$ is the number of edges of $G$ with $v$ as an endpoint; it is the degree of the vertex $v$.

Theorem 1.2. Let $G=(V, E)$ be a connected simple graph that contains at least one cycle of even ${ }^{2}$ length. Use the abbreviated notation $K:=K$ (line $G$ ), and let $p$ be a prime for which the quantity $k(p) \geq 1$.

Then for $G$ bipartite, one has

$$
K / p^{k(p)} K \cong \mathbb{Z}_{p^{k(p)}}^{\beta(G)-1} \oplus \mathbb{Z}_{\operatorname{gcd}\left(p^{k},|V|\right)},
$$

while for $G$ nonbipartite, one has

$$
K / p^{k(p)} K \cong \mathbb{Z}_{p^{k(p)}}^{\beta(G)-2} \oplus \begin{cases}0 & \text { if } p \text { is odd, } \\ \mathbb{Z}_{2}^{2} & \text { if } p=2 \text { and }|V| \text { is even, } \\ \mathbb{Z}_{4} & \text { if } p=2 \text { and }|V| \text { is odd. }\end{cases}
$$

1.3. The regularity hypothesis. Our third class of main results deals with the situation where line $G$ is regular, that is, all its vertices have the same degree. Say that a graph is $d$-regular if all of its vertices have degree $d$. It is an easy exercise to check that, for connected graphs $G$, one has line $G$ regular only in these two situations:

- $G$ itself is $d$-regular. In this case, line $G$ will be $(2 d-2)$-regular.

- $G$ is bipartite and $\left(d_{1}, d_{2}\right)$-semiregular, meaning that its vertex bipartition $V=$ $V_{1} \sqcup V_{2}$ has all vertices in $V_{i}$ of degree $d_{i}$ for $i=1,2$. In this case, line $G$ will be $\left(d_{1}+d_{2}-2\right)$-regular.

\footnotetext{
${ }^{2}$ This even length cycle need not be minimal. For example, a connected graph with two cycles $C_{1}, C_{2}$ of odd length will also contain a cycle of even length that traverses $C_{1}$, follows a path from $C_{1}$ to $C_{2}$, then traverses $C_{2}$ and follows the same path back to $C_{1}$.
} 
Two classical theorems of graph spectra explain how the the numbers of spanning trees $\kappa(G)$ and $\kappa$ (line $G$ ) determine each other in this situation. The first is due originally to Vahovskii [19] and later Kelmans [11], then rediscovered by Sachs [7, §2.4], while the second is due originally to Cvetković $[15, \S 5.2]$ (see also [14, Theorem 3.9]).

Theorem. Let $G$ be a connected graph with line $G$ regular.

(Sachs) If $G$ is d-regular, then

$$
\kappa(\text { line } G)=d^{\beta(G)-2} 2^{\beta(G)} \kappa(G) .
$$

(Cvetković) If $G$ is bipartite and $\left(d_{1}, d_{2}\right)$-semiregular, then

$$
\kappa(\text { line } G)=\frac{\left(d_{1}+d_{2}\right)^{\beta(G)}}{d_{1} d_{2}}\left(\frac{d_{1}}{d_{2}}\right)^{\left|V_{2}\right|-\left|V_{1}\right|} \kappa(G) .
$$

These results suggest a close relationship between the critical groups $K(G)$ and $K$ (line $G$ ) in both of these situations.

1.3.1. Regular graphs. We focus first on such a relation underlying Sachs' equation (2), as here one can be quite precise.

The occurrence of the factor $2^{\beta(G)} \kappa(G)$ within (2) suggests consideration of the edge subdivision graph sd $G$, obtained from $G$ placing a new vertex at the midpoint of every edge of $G$. It is well-known that

$$
\kappa(\operatorname{sd} G)=2^{\beta(G)} \kappa(G)
$$

due to an obvious $2^{\beta(G)}$-to-1 surjective map from the spanning trees of $\operatorname{sd} G$ to those of $G$. Underlying this relation, Lorenzini [12] observed that the critical groups $K(\operatorname{sd} G)$ and $K(G)$ also determine each other in a trivial way: $K(G)$ has the form given in (1) if and only if for the same positive integers $d_{1}, d_{2}, \ldots, d_{\beta(G)}$ one has the following form for $K(\operatorname{sd} G)$ :

$$
K(\operatorname{sd} G)=\bigoplus_{i=1}^{\beta(G)} \mathbb{Z}_{2 d_{i}} .
$$

See Proposition 3.2 below. In light of (4), one might expect that equation (2) generalizes to a short exact sequence of the form

$$
0 \rightarrow \mathbb{Z}_{d}^{\beta(G)-2} \rightarrow K(\text { line } G) \rightarrow K(\operatorname{sd} G) \rightarrow 0
$$

where $\mathbb{Z}_{d}$ denotes a cyclic group of order $d$. This is never far from the truth. After reviewing and developing some theory of critical groups and their functoriality in Sections 2 and 3 below, we use functoriality to prove the following result in Section 6 .

Theorem 1.3. For any connected $d$-regular simple graph $G$ with $d \geq 3$, there is a natural group homomorphism $f: K($ line $G) \rightarrow K(\mathrm{sd} G)$ whose kernel-cokernel exact sequence takes the form

$$
0 \rightarrow \mathbb{Z}_{d}^{\beta(G)-2} \oplus C \rightarrow K(\text { line } G) \stackrel{f}{\rightarrow} K(\mathrm{sd} G) \rightarrow C \rightarrow 0
$$

in which the cokernel $C$ is the following cyclic d-torsion group:

$$
C= \begin{cases}0 & \text { if } G \text { is non-bipartite and } d \text { is odd }, \\ \mathbb{Z}_{2} & \text { if } G \text { is non-bipartite and } d \text { is even }, \\ \mathbb{Z}_{d} & \text { if } G \text { is bipartite. }\end{cases}
$$


It turns out that Theorems 1.1 and 1.2 interact very well with Theorem 1.3. When $G$ is a $d$-regular simple 2-edge-connected graph, Theorem 1.1 implies that $K$ (line $G$ ) needs at most $\beta(G)$ generators, while Proposition 3.2 implies that $K(\mathrm{sd} G)$ requires at least $\beta(G)$ generators, forcing $K$ (line $G$ ) to require either $\beta(G)-1$ or $\beta(G)$ generators. This shows that the exact sequence in Theorem 1.3 is about as far as possible from being split, and gives it extra power in determining the structure of $K$ (line $G$ ) given that of $K(G)$ (and hence also $K(\operatorname{sd} G))$.

Even more precisely, it will be shown in Section 7 that when $G$ is both $d$-regular and nonbipartite, Theorems 1.2 and 1.3 combined show that $K(G)$ and $K$ (line $G$ ) determine each other uniquely in the following fashion.

Corollary 1.4. For $G$ a simple, connected, $d$-regular graph with $d \geq 3$ which is nonbipartite, after uniquely expressing

$$
K(G) \cong \bigoplus_{i=1}^{\beta(G)} \mathbb{Z}_{d_{i}}
$$

with $d_{i}$ dividing $d_{i+1}$, one has

$$
K(\text { line } G) \cong\left(\begin{array}{ll}
\beta(G)-2 \\
\bigoplus_{i=1} \mathbb{Z}_{2 d d_{i}}
\end{array}\right) \oplus \begin{cases}\mathbb{Z}_{2 d_{\beta(G)-1}} \oplus \mathbb{Z}_{2 d_{\beta(G)}} & \text { for }|V| \text { even } \\
\mathbb{Z}_{4 d_{\beta(G)-1}} \oplus \mathbb{Z}_{d_{\beta(G)}} & \text { for }|V| \text { odd. }\end{cases}
$$

1.3.2. Semiregular bipartite graphs. Section 8 uses functoriality to prove the following result analogous to Theorem 1.3 and suggested by Cvetković's equation (3).

Theorem 1.5. Let $G$ be a connected bipartite $\left(d_{1}, d_{2}\right)$-semiregular graph $G$. Then there is a group homomorphism

$$
K(\text { line } G) \stackrel{g}{\rightarrow} K(G)
$$

whose kernel-cokernel exact sequence

$$
0 \rightarrow \operatorname{ker}(g) \rightarrow K(\text { line } G) \stackrel{g}{\rightarrow} K(G) \rightarrow \operatorname{coker}(g) \rightarrow 0
$$

has coker $(g)$ all $\operatorname{lcm}\left(d_{1}, d_{2}\right)$-torsion, and has $\operatorname{ker}(g)$ all $\frac{d_{1}+d_{2}}{\operatorname{gcd}\left(d_{1}, d_{2}\right)} \operatorname{lcm}\left(d_{1}, d_{2}\right)$-torsion .

Note that this result describes the kernels and cokernels less completely than Theorem 1.3. Section 8 discusses examples illustrating why this is necessarily the case.

Section 9 illustrates some of the preceding results by showing how they apply to the examples of complete graphs and complete bipartite graphs, as well as the 1-skeleta of $d$-dimensional cubes and the Platonic solids.

\section{Some theORY OF LATTICES}

This section recalls some of the theory of rational lattices in Euclidean spaces and their determinant groups, along with functoriality and Pontrjagin duality for these groups, borrowing heavily from Bacher, de la Harpe, and Nagnibeda [1] and Treumann [18]. In the next section, these constructions will be specialized to critical groups of graphs.

2.1. Rational orthogonal decompositions. Consider $\mathbb{R}^{m}$ with its usual inner product $\langle\cdot, \cdot\rangle$ in which the standard basis vectors $e_{1}, \ldots, e_{m}$ are orthonormal. The $\mathbb{Z}$-span of this basis is the integer lattice $\mathbb{Z}^{m}$.

Definition 2.1. A rational orthogonal decomposition is an orthogonal $\mathbb{R}$-vector space decomposition of $\mathbb{R}^{m}=B^{\mathbb{R}} \oplus Z^{\mathbb{R}}$ in which $B^{\mathbb{R}}, Z^{\mathbb{R}}$ are $\mathbb{R}$-subspaces which are rational, that is, spanned by elements of $\mathbb{Z}^{m}$. 
Example 2.2. The main example of interest for us will be the following, discussed further in Section 3. If $G=(V, E)$ is a graph with $|E|=m$, then the space $Z^{\mathbb{R}}$ of 1 -cycles together with its orthogonal complement, the space $B^{\mathbb{R}}$ of bonds or 1-coboundaries, give a rational orthogonal decomposition $\mathbb{R}^{E} \cong \mathbb{R}^{m}=B^{\mathbb{R}} \oplus Z^{\mathbb{R}}$. Here one must fix an (arbitrary) orientation of the edges in $E$ in order to make the identification $\mathbb{R}^{E} \cong \mathbb{R}^{m}$. In the remaining sections, the basis element of $\mathbb{R}^{E}$ corresponding to an edge $\{u, v\}$ of $G$ oriented from $u$ to $v$ will sometimes be denoted $e$ and sometimes $(u, v)$, with the convention that $(v, u)=-(u, v)=-e$ in $\mathbb{R}^{E}$.

An $r$-dimensional rational subspace $\Lambda^{\mathbb{R}} \subset \mathbb{R}^{m}$ inherits the inner product $\langle\cdot, \cdot\rangle$. The space $\Lambda^{\mathbb{R}}$ contains two lattices of rank $r$, namely $\Lambda:=\Lambda^{\mathbb{R}} \cap \mathbb{Z}^{m}$ and its dual lattice

$$
\Lambda^{\#}:=\left\{x \in \Lambda^{\mathbb{R}}:\langle x, \lambda\rangle \in \mathbb{Z} \text { for all } \lambda \in \Lambda\right\} .
$$

Since $\langle\Lambda, \Lambda\rangle \subset\left\langle\mathbb{Z}^{m}, \mathbb{Z}^{m}\right\rangle=\mathbb{Z}$, one has an inclusion $\Lambda \subset \Lambda^{\#}$. Their quotient is called the determinant group

$$
\operatorname{det}(\Lambda):=\Lambda^{\#} / \Lambda \text {. }
$$

Given a rational orthogonal decomposition $\mathbb{R}^{m}=B^{\mathbb{R}} \oplus Z^{\mathbb{R}}$, one obtains two determinant groups $\operatorname{det}(B), \operatorname{det}(Z)$, which turn out to be both isomorphic to what we will call the critical group

$$
K:=\mathbb{Z}^{m} /(B \oplus Z)
$$

of the rational orthogonal decomposition. Indeed, if $\pi_{B}, \pi_{Z}$ denote the orthogonal projections from $\mathbb{R}^{m}$ onto $B^{\mathbb{R}}, Z^{\mathbb{R}}$, then these maps turn out to give rise to surjections from $\mathbb{Z}^{m}$ onto $B^{\#}$ and $Z^{\#}$, respectively, and which induce isomorphisms (see [1, Proposition 3])

$$
\begin{array}{lcccc}
\operatorname{det}(B) & \cong & K & \cong & \operatorname{det}(Z) \\
B^{\#} / B & \stackrel{\pi_{B}}{\leftrightarrows} & \mathbb{Z}^{m} /(B \oplus Z) & \stackrel{\pi_{Z}}{\longrightarrow} & Z^{\#} / Z .
\end{array}
$$

One can compute the critical group $K$ very explicitly as the (integer) cokernel of several matrices, for example via their Smith normal form. If the lattices $B, Z$ have $\mathbb{Z}$-bases $\left\{b_{1}, \ldots, b_{\alpha}\right\},\left\{z_{1}, \ldots, z_{\beta}\right\}$ then let $M_{B}, M_{Z}, M_{B \oplus Z}$ be matrices having columns given by $\left\{b_{i}\right\}_{i=1}^{\alpha},\left\{z_{j}\right\}_{j=1}^{\beta},\left\{b_{i}\right\}_{i=1}^{\alpha} \cup\left\{z_{j}\right\}_{j=1}^{\beta}$, respectively. The Gram matrices $M_{B}^{t} M_{B}, M_{Z}^{t} M_{Z}$ express the bases for $B, Z$ in terms of the dual bases for $B^{\#}, Z^{\#}$, and hence

$$
\begin{aligned}
K & \cong \mathbb{Z}^{m} /(B \oplus Z)=\operatorname{coker} M_{B \oplus Z}, \\
& \cong B^{\#} / B=\operatorname{coker}\left(M_{B}^{t} M_{B}\right), \\
& \cong Z^{\#} / Z=\operatorname{coker}\left(M_{Z}^{t} M_{Z}\right) .
\end{aligned}
$$

2.2. Functoriality. Suppose that one has two rational orthogonal decompositions $\mathbb{R}^{m_{i}}=$ $B_{i}^{\mathbb{R}} \oplus Z_{i}^{\mathbb{R}}$ for $i=1,2$, and an $\mathbb{R}$-linear map $f: \mathbb{R}^{m_{1}} \rightarrow \mathbb{R}^{m_{2}}$. When does $f$ induce a homomomorphism $f: K_{1} \rightarrow K_{2}$ between their critical groups?

It is natural to assume that $f$ carries the integer lattice $\mathbb{Z}^{m_{1}}$ into $\mathbb{Z}^{m_{2}}$, that is, $f$ is represented by a matrix in $\mathbb{Z}^{m_{2} \times m_{1}}$. Note that this already implies that the adjoint map $f^{t}: \mathbb{R}^{m_{2}} \rightarrow \mathbb{R}^{m_{1}}$ with respect to the standard inner products will also satisfy $f^{t}\left(\mathbb{Z}^{m_{2}}\right) \subset \mathbb{Z}^{m_{1}}$, since this map is represented by the transposed $\mathbb{Z}^{m_{1} \times m_{2}}$ matrix.

What one needs further to induce homomorphisms of critical groups is that $f\left(B_{1}\right) \subset B_{2}$ and $f\left(Z_{1}\right) \subset Z_{2}$. The following proposition gives a useful reformulation.

Proposition 2.3. For a linear map $f: \mathbb{R}^{m_{1}} \rightarrow \mathbb{R}^{m_{2}}$ satisfying $f\left(\mathbb{Z}^{m_{1}}\right) \subset \mathbb{Z}^{m_{2}}$, one has

$$
f\left(B_{1}\right) \subset B_{2} \Longleftrightarrow f^{t}\left(Z_{2}\right) \subset Z_{1} \Longleftrightarrow f\left(Z_{1}\right) \subset Z_{2}^{\#}
$$


and

$$
f\left(Z_{1}\right) \subset Z_{2} \Longleftrightarrow f^{t}\left(B_{2}\right) \subset B_{1} \Longleftrightarrow f\left(B_{1}\right) \subset B_{2}^{\#} .
$$

Proof. All of the implications follow using the adjointness of $f, f^{t}$ with respect to the pairings on $\mathbb{R}^{m_{1}}, \mathbb{R}^{m_{2}}$, along with the definitions of $B_{i}^{\#}, Z_{i}^{\#}$ and the fact that $B_{i}=Z_{i}^{\perp}$.

When a linear map $f: \mathbb{R}^{m_{1}} \rightarrow \mathbb{R}^{m_{2}}$ satisfies all of the conditions in the previous proposition, we say that $f$ is a morphism of rational orthogonal decompositions. It is clear that $f$ then induces a homomorphism $K_{1} \rightarrow K_{2}$ between the critical groups, denoted here also by $f$.

Note the following property of such maps $f$ for future use.

Proposition 2.4. Any morphism $f: \mathbb{R}^{m_{1}} \rightarrow \mathbb{R}^{m_{2}}$ of rational orthogonal decompositions intertwines the projection maps onto either $B_{i}^{\mathbb{R}}$ or $Z_{i}^{\mathbb{R}}$. That is, the following diagram commutes:

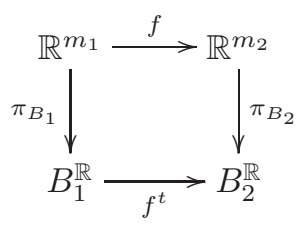

and the same holds replacing $B_{i}$ by $Z_{i}$ everywhere.

Proof. Given $x_{1} \in \mathbb{R}^{m_{1}}$ and $b_{2} \in B_{2}^{\mathbb{R}}$, note that

$$
\left\langle\pi_{B_{2}}\left(f\left(x_{1}\right)\right), b_{2}\right\rangle=\left\langle f\left(x_{1}\right), b_{2}\right\rangle=\left\langle x_{1}, f^{t}\left(b_{2}\right)\right\rangle=\left\langle\pi_{B_{1}}\left(x_{1}\right), f^{t}\left(b_{2}\right)\right\rangle=\left\langle f\left(\pi_{B_{1}}\left(x_{1}\right)\right), b_{2}\right\rangle .
$$

Since this equality holds for any test vector $b_{2} \in B_{2}^{\mathbb{R}}$, one concludes that $\pi_{B_{2}}\left(f\left(x_{1}\right)\right)=$ $f\left(\pi_{B_{1}}\left(x_{1}\right)\right)$.

2.3. Pontrjagin duality. Every finite abelian group $K$ is isomorphic to its Pontrjagin dual

$$
K^{*}:=\operatorname{Hom}_{\mathbb{Z}}(K, \mathbb{Q} / \mathbb{Z}) .
$$

This isomorphism is not, in general, natural (although the isomorphism $K \cong K^{* *}$ is). However, for critical groups $K=\mathbb{Z}^{m} /(B \oplus Z)$ associated with a rational orthogonal decomposition, the isomorphism comes about naturally from the pairing

$$
\begin{array}{ccc}
\mathbb{Z}^{m} \times \mathbb{Z}^{m} & \rightarrow & \mathbb{Q} \\
(x, y) & \mapsto & \langle\pi(x), \pi(y)\rangle
\end{array}
$$

where $\pi$ is either of the orthogonal projections $\pi_{B}$ or $\pi_{Z}$. This induces a pairing

$$
\langle\cdot, \cdot\rangle: K \times K \rightarrow \mathbb{Q} / \mathbb{Z}
$$

which is nondegenerate in the sense that the following map is an isomorphism:

$$
\begin{array}{cccc}
K & \rightarrow & \operatorname{Hom}_{\mathbb{Z}}(K, \mathbb{Q} / \mathbb{Z}) & \left(=K^{*}\right) \\
x & \mapsto & \langle x, \cdot\rangle . &
\end{array}
$$

Pontrjagin duality is contravariant in the following sense. Given a homomorphism $f$ : $K_{1} \rightarrow K_{2}$ of abelian groups, there is a dual morphism $f^{*}: K_{2}^{*} \rightarrow K_{1}^{*}$ given by $f^{*}(g)=g \circ f$. The next proposition asserts that this duality interacts as one would expect with morphisms of rational orthogonal decompositions. 
Proposition 2.5. For a morphism $f: \mathbb{R}^{m_{1}} \rightarrow \mathbb{R}^{m_{2}}$ of rational orthogonal decompositions, Pontrjagin duality identifies $f^{t}$ with $f^{*}$, in the sense that the following diagram commutes:

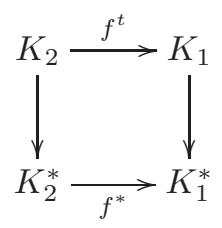

Here the vertical maps are both Pontrjagin duality isomorphisms as in (9).

Proof. Unravelling the definitions, this amounts to checking that if $x_{i} \in \mathbb{Z}^{m_{i}}$ for $i=1,2$, then one has $\left\langle f^{t}\left(x_{2}\right), x_{1}\right\rangle=\left\langle x_{2}, f\left(x_{1}\right)\right\rangle$.

This last proposition has a useful consequence.

Corollary 2.6. For a morphism $f: \mathbb{R}^{m_{1}} \rightarrow \mathbb{R}^{m_{2}}$ of rational orthogonal decompositions, the maps induced by $f, f^{t}$ on critical groups satisfy $\operatorname{ker}(f)^{*} \cong \operatorname{coker}\left(f^{t}\right)$ and $\operatorname{coker}(f)^{*} \cong \operatorname{ker}\left(f^{t}\right)$.

Proof. Pontrjagin duality generally gives $\operatorname{ker}(f)^{*} \cong \operatorname{coker}\left(f^{*}\right)$ and $\operatorname{coker}(f)^{*} \cong \operatorname{ker}\left(f^{*}\right)$, so this follows from Proposition 2.5.

\section{The CRITICAL GROUP OF A GRAPH}

This section particularizes the discussion of critical groups from the previous section to the context of Example 2.2, that is, the critical group $K(G)$ for a graph $G=(V, E)$. It also recalls how one can use spanning trees/forests to be more explicit about some of these constructions, and reviews for later use some other known results about critical groups of graphs. Note that we will use the term "spanning tree" when discussing connected graphs and "spanning forest" when no connectivity is assumed.

3.1. Cycles, bonds, Laplacians, and spanning trees. Let $G=(V, E)$ be a graph. After picking an orientation for its edges, the usual cellular boundary map from 1-chains to 0 -chains with real or integer coefficients

$$
\begin{aligned}
& \mathbb{R}^{E} \stackrel{\partial_{G}}{\longrightarrow} \mathbb{R}^{V} \\
& \mathbb{Z}^{E} \stackrel{\partial_{G}}{\longrightarrow} \mathbb{Z}^{V}
\end{aligned}
$$

is defined $\mathbb{R}$ - or $\mathbb{Z}$-linearly as follows: A basis element $e$ corresponding to an edge directed from vertex $u$ to vertex $v$ is sent to $\partial_{G}(e)=+v-u$. One considers the negative $-e$ of this basis element as representing the same edge but directed from $v$ to $u$, which is consistent with

$$
\partial_{G}(-e)=+u-v=-\partial_{G}(e) .
$$

Elements in the kernel of $Z^{\mathbb{R}}:=\operatorname{ker} \partial_{G}$ are called cycles, while elements in the perpendicular space $B^{\mathbb{R}}:=\operatorname{im} \partial_{G}^{t}$ are called bonds. Thus $\mathbb{R}^{E}=B^{\mathbb{R}} \oplus Z^{\mathbb{R}}$ is a rational orthogonal decomposition associated with the graph $G=(V, E)$, and we denote by $K(G)$ the associated critical group.

The lattice $B$ of bonds is known to be spanned by the signed incidence vectors $b\left(V_{1}, V_{2}\right)$ of the directed edges that span across a cut (partition) $V=V_{1} \sqcup V_{2}$. The lattice $Z$ of cycles is known to be spanned by the signed incidence vectors $z(C)$ coming from directed cycles in $G$. 
If one wants a smaller $\mathbb{Z}$-spanning set for $B$, one can take the vectors $b_{G}(\{v\}, V-\{v\})$ for cuts that isolate single vertices; this vector $b_{G}(\{v\}, V-\{v\})$ is exactly the row vector of the $|V| \times|E|$ boundary map $\partial_{G}$ indexed by $v$. To simplify notation, we will write

$$
b_{G}(v):=b_{G}(\{v\}, V \backslash\{v\})
$$

for this bond, and we will call it the bond at $v$ in $G$. In order to select out of this spanning set a $\mathbb{Z}$-basis for $B$, one should omit exactly one vertex from each connected component of $G$.

Here are a few consequences of these facts:

(i) The Gram matrix $M_{B}^{t} M_{B}$ corresponding to the above mentioned $\mathbb{Z}$-basis for $B$ gives what is usually called a (reduced) Laplacian matrix $\overline{L(G)}$; the matrix $M_{B}$ is obtained from $\partial_{G}^{t}$ by removing the columns corresponding to the chosen vertex in each connected component of $G$. As a consequence, one has by Kirchhoff's Matrixtree Theorem (see, e.g., [20, Theorem 2.2.12]) that

$$
|K(G)|=\operatorname{det} \overline{L(G)}=\kappa(G),
$$

the number of spanning forests in $G$.

(ii) (The chip-firing/dollar-game/sandpile/Picard presentations for $K(G)$ )

Given a connected graph $G=(V, E)$ with boundary map $\mathbb{Z}^{E} \stackrel{\partial}{\longrightarrow} \mathbb{Z}^{V}$, bond lattice $B:=\operatorname{im} \partial^{t}$, and any vertex $v_{0}$ in $V$, one has an isomorphism

$$
\begin{aligned}
& K(G) \cong \operatorname{coker}\left(M_{B}^{t} M_{B}\right) \\
& \cong \operatorname{coker}(\overline{L(G)}) \\
& \cong \mathbb{Z}^{V \backslash\left\{v_{0}\right\}} / \overline{L(G)} \\
& \cong \mathbb{Z}^{V} /\left(\mathbb{Z} v_{0}+\operatorname{im}\left(\partial_{G} \partial_{G}^{t}\right)\right) \\
& \cong \mathbb{Z}^{V} /\left(\mathbb{Z} v_{0}+\partial_{G}(B)\right) \\
& \cong \mathbb{Z}^{V} /\left(\mathbb{Z} v_{0}+\mathbb{Z}\left(\partial_{G} b_{G}(v)_{v \in V}\right)\right) .
\end{aligned}
$$

(iii) For any vertex $u$ of $G$, one has the relation

$$
\sum_{\{v \in V:\{u, v\} \in E\}}(u, v)=0
$$

in $K(G)=\mathbb{Z}^{E} /(B \oplus Z)$.

(iv) For any directed cycle $u_{0} \rightarrow u_{1} \rightarrow \cdots \rightarrow u_{\ell-1} \rightarrow u_{\ell}=u_{0}$ in $G$, one has the relation

$$
\sum_{i=0}^{\ell-1}\left(u_{i}, u_{i+1}\right)=0
$$

in $K(G)=\mathbb{Z}^{E} /(B \oplus Z)$.

Fixing a particular spanning forest $T$ for $G$ allows one to simultaneously construct $\mathbb{Z}$ bases of $B$ and $Z$. Removing any edge $e$ in the forest $T$ creates a new connected component in the forest, say with vertex set $V_{e} \subset V$; ranging over all edges $e$ in $T$, the signed incidence vectors $b_{e}^{T}$ for the cuts $V=V_{e} \sqcup\left(V-V_{e}\right)$ form a $\mathbb{Z}$-basis for $B$. Dually, adding any edge $e$ in $E-T$ to $T$ creates a unique cycle in $T \cup\{e\}$; ranging over all edges in $E-T$, the signed incidence vectors $z_{e}^{T}$ of these cycles form a $\mathbb{Z}$-basis for $Z$. 

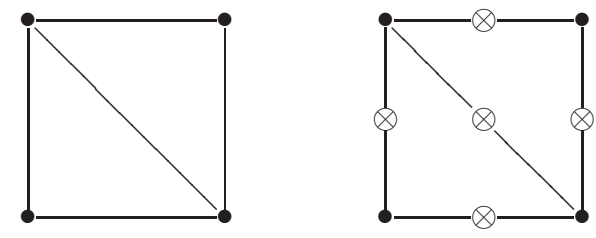

Figure 2. A graph $G$ and its edge subdivision sd $G$.

3.2. A presentation for $K($ line $G)$. Proposition 3.1 below gives a useful presentation for $K$ (line $G$ ) that is an immediate consequence of the last equation in assertion (3.1)(ii) above. It will be used both in the proof of Theorem 1.1 and in the analysis of $K$ (line $K_{n}$ ) in Section 9.1.

Let $G=(V, E)$ be a connected simple graph, so that line $G=\left(V_{\text {line } G}, E_{\text {line } G}\right)$ is also connected. Identify the vertex set $V_{\text {line }} G$ of the line graph of $G$ with the edge set $E$ of $G$. After picking arbitrary orientations for the edges of line $G$, consider the boundary map for line $G$ :

$$
\mathbb{Z}^{E_{\text {line } G} \longrightarrow \mathbb{Z}^{E}}\left(=\mathbb{Z}^{\left.V_{\text {line } G}\right)} .\right.
$$

Proposition 3.1. Given a connected simple graph $G=(V, E)$ and any edge $e_{0}$ in $E$, one has an isomorphism

$$
\begin{aligned}
K(\text { line } G) & \cong \mathbb{Z}^{E} /\left(\mathbb{Z} e_{0}+\partial_{\text {line } G}\left(B_{\text {line } G}\right)\right) \\
& \cong \mathbb{Z}^{E} /\left(\mathbb{Z} e_{0}+\mathbb{Z}\left(\partial_{\text {line } G} b_{\text {line } G}(e)_{e \in E}\right)\right) .
\end{aligned}
$$

3.3. Lorenzini's result on edge subdivisions. The edge subdivision of a graph $G$ is the graph sd $G$ obtained by creating a new midpoint vertex called $u v$ for every edge $\{u, v\}$ of $G$; that is, $\{u, v\}$ is removed and replaced by two edges $\{u, u v\},\{v, u v\}$ in sd $G$. In [12] Lorenzini first observed that the critical groups $K(\operatorname{sd} G)$ and $K(G)$ determine each other in a trivial way, using the description $K=Z^{\#} / Z$. If $\left\{C_{1}, \ldots, C_{\beta}\right\}$ is any set of directed cycles in $G$ whose incidence vectors $\left\{z\left(C_{i}\right)\right\}_{i=1}^{\beta}$ give a $\mathbb{Z}$-basis for $Z_{G}$, then one can subdivide those same cycles to obtain a $\mathbb{Z}$-basis $\left\{z_{\mathrm{sd} C_{i}}\right\}_{i=1}^{\beta}$ for $Z_{\mathrm{sd} G}$. One then checks that

$$
\left\langle z_{\text {sd } C_{i}}, z_{\text {sd } C_{j}}\right\rangle=2\left\langle z\left(C_{i}\right), z\left(C_{j}\right)\right\rangle
$$

for each $i, j$. Hence one has the following relation between their Gram matrices:

$$
M_{\mathrm{sd} G}^{t} M_{\mathrm{sd} G}=2 M_{G}^{t} M_{G}
$$

and the following simple relation between their cokernels, the critical groups:

Proposition 3.2 (Lorenzini [12]). Let $G$ be a graph with $\beta$ independent cycles. Expressing $K(G) \cong \bigoplus_{i=1}^{\beta} \mathbb{Z}_{d_{i}}$ for positive integers $d_{1}, d_{2}, \ldots, d_{\beta} \geq 1$, one has $K(\operatorname{sd} G) \cong \bigoplus_{i=1}^{\beta} \mathbb{Z}_{2 d_{i}}$.

It will be useful later to have an expression of this result in terms of explicit morphisms (as was done also in [12]). Consider the pair of adjoint maps defined $\mathbb{R}$-linearly by

$$
\begin{array}{ccc}
\mathbb{R}^{E_{\mathrm{sd} G}} & \stackrel{h}{\longmapsto} & \mathbb{R}^{E_{G}} \\
(u, u v) & \longmapsto & (u, v) \\
(u v, v) & \longmapsto & (u, v) \\
& & \\
\mathbb{R}^{E_{G}} & \stackrel{h^{t}}{\longmapsto} & \mathbb{R}^{E_{\mathrm{sd} G}} \\
(u, v) & \longmapsto & (u, u v)+(u v, v) .
\end{array}
$$


One can easily check that these are morphisms of rational orthogonal decompositions, and hence give rise to a morphism $h: K(\mathrm{sd} G) \rightarrow K(G)$ of critical groups. The relation (11) between the two $\beta \times \beta$ Gram matrices shows that the kernel-cokernel exact sequence associated to $h$ takes this form:

$$
\begin{aligned}
& 0 \longrightarrow \operatorname{ker}(h) \quad \longrightarrow \quad K(\operatorname{sd} G) \quad \stackrel{h}{\longrightarrow} K(G) \quad \longrightarrow 0 \\
& 0 \longrightarrow \mathbb{Z}_{2}^{\beta} \longrightarrow \bigoplus_{i=1}^{\beta} \mathbb{Z}_{2 d_{i}} \longrightarrow \bigoplus_{i=1}^{\beta} \mathbb{Z}_{d_{i}} \longrightarrow 0 .
\end{aligned}
$$

Proposition 3.2 is equivalent to the assertion that $K(\operatorname{sd} G)$ can be generated by $\beta$ elements and fits into an exact sequence of this form, generalizing equation (4) from the Introduction.

3.4. A non-standard treatment of the complete graph. Let $K_{n}$ be the complete graph on $n$ vertices. A celebrated formula of Cayley asserts that $\kappa\left(K_{n}\right)=n^{n-2}$ (see, e.g., [8, Section 13.2]). Generalizing this to compute the critical group $K\left(K_{n}\right)$ is a favorite example of many papers in the subject. We approach this calculation in a slightly nonstandard way here, mainly because it will provide us with a crucial technical lemma for later use in Section 6.4.

Proposition 3.3. The complete graph $K_{n}$ has critical group

$$
K\left(K_{n}\right) \cong \mathbb{Z}_{n}^{n-2}
$$

Furthermore, in the presentation $K\left(K_{n}\right)=\mathbb{Z}^{E} /(B \oplus Z)$, a minimal generating set is provided by the images of any set of $n-2$ edges which form a spanning tree connecting $n-1$ out of the $n$ vertices.

Proof. Since Cayley's formula implies $\left|K\left(K_{n}\right)\right|=\left|\mathbb{Z}_{n}^{n-2}\right|$, it will suffice to show that $K\left(K_{n}\right)$ is all $n$-torsion and that it can be generated by $n-2$ elements as in the second assertion. Let $[n]:=\{1,2, \ldots, n\}$ denote the vertex set $V$ for $K_{n}$.

To show $K\left(K_{n}\right)$ is all $n$-torsion, given any directed edge $e=(i, j)$ in $K_{n}$, we will prove that $n \cdot e$ is equal to a sum of cycles and bonds. Indeed, we can take the sum of the directed cycles $(i, j)+(j, k)+(k, i)$ for $k \in[n]-\{i, j\}$, and add the two bonds

$$
\begin{gathered}
b(\{i\},[n]-\{i\})=(i, 1)+(i, 2)+\cdots+(i, n) \\
b([n]-\{j\},\{j\})=(1, j)+(2, j)+\cdots+(n, j) .
\end{gathered}
$$

For the second assertion, let $T$ be a collection of $n-2$ edges that form a spanning tree connecting $n-1$ out of the $n$ vertices. By symmetry, we may assume that $n$ is the vertex that is isolated by $T$. The edges of $K_{n}$ can be partitioned into two sets, $E\left(K_{n-1}\right)$ and $\{(i, n)\}_{i=1}^{n-1}$.

Any edge $e$ in $E\left(K_{n-1}\right)$ either lies in $T$ or forms a cycle $z\left(C_{T, e}\right)$ that lets one express $e$ in terms of the elements of $T$ modulo $Z\left(K_{n-1}\right)$, and hence modulo $Z=Z\left(K_{n}\right)$.

For each $1 \leq i \leq n-1$, the bond

$$
b_{i}:=\sum_{\substack{j=1 \\ j \neq i}}^{n}(i, j) \equiv 0 \quad \bmod B,
$$

and it follows that

$$
(i, n) \equiv-\sum_{\substack{j=1 \\ j \neq i}}^{n-1}(i, j) \quad \bmod B .
$$


The edges in the sum all belong to $K_{n-1}$ and thus, according to the previous paragraph, can be written in terms of $T$ modulo $Z$. It follows that $(i, n)$ can be written in terms of $T$ modulo $B+Z$.

\section{Proof of Theorem 1.1}

Recall the statement of Theorem 1.1:

Theorem 1.1. When the simple graph $G$ is 2-edge-connected, the critical group $K$ (line $G$ ) can be generated by $\beta(G)$ elements.

The $\beta(G)$ generators will come from the set of edges in the complement $E \backslash T$ of a carefully chosen spanning tree $T$ for $G$.

Definition 4.1. For a connected graph $G=(V, E)$, say that a spanning tree $T \subset E$ for $G$ has an absorption order in $G$ if one can linearly order the union $V \sqcup T$ of its vertices and edges in the following way:

(i) The order begins with a vertex $v_{0}$ in $V$ followed by an edge $e_{0}$ of $T$, such that $e_{0}$ is the unique edge of $T$ incident to $v_{0}$ (so $v_{0}$ is a leaf-vertex of $T$ attached along the leaf-edge $e_{0}$ ).

(ii) For every other vertex $v$ in $V \backslash\left\{v_{0}\right\}$, there exists an edge $e=\{v, w\}$ such that $w$ occurs earlier in the order than $v$, and the edge $e$ either lies in $E \backslash T$ or occurs earlier in the order than $v$.

(iii) For every other edge $e$ in $T \backslash\left\{e_{0}\right\}$, there exists a vertex $v$ incident to $e$ which occurs earlier in the order than $e$, and every other edge incident to $v$ either lies in $E \backslash T$ or occurs earlier in the order than $e$.

The relevance of an absorption order for a spanning tree is given by the algebraic consequence in the following proposition. Say that an orientation of the edges of a tree $T$ is bipartite if, for every vertex $v$, the edges of $T$ incident to $v$ are either all oriented toward $v$ or all oriented away from $v$.

Proposition 4.2. Let $G=(V, E)$ be a simple graph, and assume it has a spanning tree $T \subset E$ which has an absorption order in $G$.

Then the images of the basis elements in $\mathbb{Z}^{E}$ corresponding to the edges $E \backslash T$ not lying on $T$ give a set of $\beta(G)$ generators for $K$ (line $G)$, using the presentation from Proposition 3.1

$$
K(\text { line } G) \cong \mathbb{Z}^{E} /\left(\mathbb{Z} e_{0}+\partial_{\text {line } G}\left(B_{\text {line } G}\right)\right),
$$

assuming that the orientation chosen for $G$ restricts to a bipartite orientation of $T$ (although line $G$ may be oriented arbitrarily), and the edge $e_{0}$ is the designated leaf-edge of $T$ appearing second in the absorption order.

To prove this, note the following crucial lemma:

Lemma 4.3. When a connected graph $G=(V, E)$ is oriented in a way that restricts to a bipartite orientation for a spanning tree $T \subset E$, then any edge $e=\{v, w\}$ has

$$
b_{G}(v) \equiv \pm b_{G}(w) \quad \bmod \quad \mathbb{Z} e+\mathbb{Z}(E \backslash T)+\partial_{\text {line } G}\left(B_{\text {line } G}\right) .
$$

Proof of Lemma 4.3. Label the edges of $G$ incident to $v$ other than $e$ by

$$
\underbrace{e_{1}, \ldots, e_{p}}_{\text {in } T}, \underbrace{e_{p+1}, e_{p+2}, \ldots, e_{P}}_{\text {in } E \backslash T}
$$


and those incident to $w$ other than $e$ by

$$
\underbrace{f_{1}, \ldots, f_{q}}_{\text {in } T}, \underbrace{f_{q+1}, f_{q+2}, \ldots, f_{Q}}_{\text {in } E \backslash T} .
$$

With these notations, one then has

$$
\begin{aligned}
\partial_{\text {line } G} b_{\text {line } G}(e) & =\left(e_{1}-e\right)+\cdots+\left(e_{P}-e\right)+\left(f_{1}-e\right)+\cdots+\left(f_{Q}-e\right) \\
& =\left(e_{1}+\cdots+e_{P}\right)+\left(f_{1}+\cdots+f_{Q}\right)-(P+Q) e
\end{aligned}
$$

Because the orientation of $G$ when restricted to $T$ is bipartite,

$$
\begin{aligned}
b_{G}(v) & = \pm\left(e_{1}+\cdots+e_{p}\right) \pm e_{p+1} \pm e_{p+2} \pm \cdots \pm e_{P} \\
b_{G}(w) & = \pm\left(f_{1}+\cdots+f_{q}\right) \pm f_{q+1} \pm f_{q+2} \pm \cdots \pm f_{Q} .
\end{aligned}
$$

Comparison of (12) and (13) shows that one of the two expressions $b_{G}(v)+b_{G}(w)$ or $b_{G}(v)-$ $b_{G}(w)$ differs from $\partial_{\text {line } G} b_{\text {line } G}(e)$ by a $\mathbb{Z}$-linear combination of the edges in

$$
\{e\} \cup\left\{e_{p+1}, e_{p+2}, \cdots, e_{P}, f_{q+1}, f_{q+2}, \cdots, f_{Q}\right\} .
$$

Since the second set in the above union lies in $E \backslash T$, the lemma follows.

Proof of Proposition 4.2. One needs to show that the subgroup of $\mathbb{Z}^{E}$ defined by

$$
I:=\mathbb{Z}(E \backslash T)+\mathbb{Z} e_{0}+\partial_{\text {line } G}\left(B_{\text {line } G}\right)
$$

is all of $\mathbb{Z}^{E}$. Since $E \backslash T$ is a subset of $I$, it is enough to show that every edge $e$ in $T$ lies in I. More strongly, one shows by induction on their location in the absorption order for $T$, that not only does every edge $e$ in $T$ lie in $I$, but also every vertex $v$ in $V$ has $b_{G}(v)$ lying in $I$.

The base case for this induction deals both with the first vertex $v_{0}$ and the first edge $e_{0}$, which come at the beginning of the absorption order. Since $v_{0}$ is a leaf vertex of $T$ along the edge $e_{0}$, one has $b_{G}\left(v_{0}\right)$ in $I$, since the only edges incident to $v_{0}$ are $e_{0}$ and edges of $E \backslash T$. For the edge $e_{0}$, note that it trivially lies in $I$.

In the inductive step, the next element in the absorption order is either a vertex $v \neq v_{0}$ or an edge $e \neq e_{0}$.

If the next element is a vertex $v \neq v_{0}$, then by Definition 4.1(ii), there exists an edge $e=\{v, w\}$ for which $b_{G}(w)$ lies in $I$ by induction, and either $e$ lies in $E \backslash T$ (so that $e$ is in $I$ ) or $e$ is earlier in the order than $v$ (so that $e$ is in $I$ by induction). Hence Lemma 4.3 shows that $b_{G}(v)$ also lies in $I$.

If the next element is an edge $e \neq e_{0}$, then by Definition 4.1(iii), there exists a vertex $v$ incident to $e$ for which $b_{G}(v)$ lies in $I$ by induction. Note that $b_{G}(v)$ is a \pm 1 combination of all the edges $e^{\prime}$ incident to $v$, and all of these other edges $e^{\prime} \neq e$ either have $e^{\prime}$ in $E \backslash T$ (so that $e^{\prime}$ is in $I$ ) or $e^{\prime}$ is earlier in the order than $e$ (so that $e^{\prime}$ is in $I$ by induction). Hence $e$ also lies in $I$.

To show that 2-edge-connected graphs $G$ always have a spanning tree $T$ with an absorption order, we recall the well-known reformulation of 2-edge-connectivity in terms of ear decompositions; see e.g., [20, Definition 4.2.7].

Definition 4.4. Let $G=(V, E)$ be a simple graph. An ear of $G$ is a walk that alternates (incident) vertices $u_{i}$ and edges $e_{i}$

$$
v:=u_{1}, e_{1}, u_{2}, e_{2}, \ldots, u_{\ell}, e_{\ell}, u_{\ell+1}:=w
$$



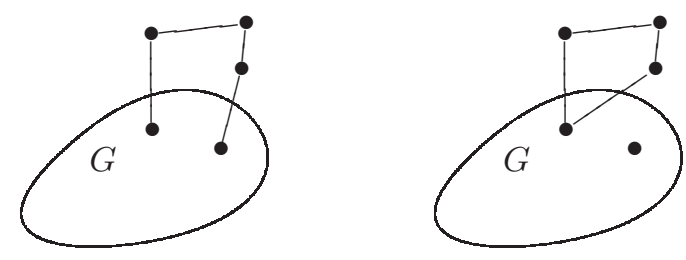

FiguRE 3. Graphs with open and closed ears.

such that the internal vertices $u_{2}, \ldots, u_{\ell}$ are each of degree 2 in $G$. If $v \neq w$, it is called an open ear (and necessarily $\ell \geq 1$ ), while if $v=w$, it is called a closed ear (and necessarily $\ell \geq 3$, because $G$ is simple).

An ear decomposition of $G$ is a decomposition of its vertices and edges

$$
P_{0} \cup P_{1} \cup \cdots \cup P_{k}
$$

such that $P_{0}$ is a cycle, and for $1 \leq i \leq k, P_{i}$ is an ear of $P_{0} \cup P_{1} \cup \cdots \cup P_{i}$.

Proposition 4.5 ([20, Theorem 4.2.8]). A graph is 2-edge-connected if and only if it has an ear decomposition.

In light of Proposition 4.2, the following result implies Theorem 1.1.

Proposition 4.6. Let $G=(V, E)$ be a simple 2-edge-connected graph. Then $G$ has at least one spanning tree $T \subset E$ with an absorption order in $G$.

Proof. Induct on the number $k$ of ears in an ear decomposition $P_{0} \cup P_{1} \cup \cdots \cup P_{k}$ for $G$.

In the base case $k=0$, the graph $G=P_{0}$ is a $n$-cycle. Label its vertices $V=$ $\left\{v_{0}, v_{1}, \ldots, v_{n-1}\right\}$ and edges $E=\left\{e_{0}, e_{1}, \ldots, e_{n-1}\right\}$ so that $e_{i}=\left\{v_{i}, v_{i+1}\right\}$ with indices taken modulo $n$. Then one can easily check that $T=\left\{e_{0}, e_{1}, \ldots, e_{n-2}\right\}$ is a spanning tree, and $\left(v_{0}, e_{0}, v_{1}, e_{1}, \ldots, v_{n-2}, e_{n-2}, v_{n-1}\right)$ is an absorption order for $T$ in $G$.

In the inductive step, one may assume that $G^{-}:=P_{0} \cup P_{1} \cup \cdots \cup P_{k-1}$ has a spanning tree $T^{-}$with an absorption order in $G^{-}$. Choose the labelling of the endpoints $v, w$ of the ear $P_{k}$ so that $v$ comes weakly earlier than $w$ in the absorption order for $T^{-}$, where the vertices and edges of $P_{k}$ are labelled as in (14). Extend $T^{-}$to

$$
T:=T^{-} \sqcup\left\{u_{2}, u_{3}, \ldots, u_{\ell}\right\}
$$

which is easily seen to be a spanning tree for $G$. One extends the absorption order for $T^{-}$ in $G^{-}$to one for $T$ in $G$ by inserting the subsequence

$$
\left(u_{2}, e_{2}, u_{3}, e_{3}, u_{4}, \ldots, u_{\ell}, e_{\ell}\right)
$$

into the absorption sequence for $T^{-}$in one of two possible locations, depending upon whether $v$ and $w$ are the initial vertex $v_{0}$ of the absorption order of $T^{-}$, or not.

First we assume that $P_{k}$ is an open ear (that is, $v \neq w$ ) or $P_{k}$ is a closed ear with $v=w \neq v_{0}$. In this case, one can check that inserting the subsequence (15) immediately after $v$ in the absorption order for $T^{-}$in $G^{-}$gives an absorption order for $T$ in $G$.

In the case that $P_{k}$ is a closed ear with $v=w=v_{0}$, one checks that inserting the subsequence (15) at the very beginning of the absorption order for $T^{-}$in $G^{-}$gives an absorption order for $T$ in $G$. Note that $u_{2}, e_{2}$ become the "new" $v_{0}, e_{0}$ in this absorption order. 


\section{Proof of Theorem 1.2}

For a prime $p$ let $k(p)$ be the largest integer such that $p^{k(p)}$ divides all of the sums $\operatorname{deg}_{G}(v)+\operatorname{deg}_{G}(w)$ as one runs through all edges $e=\{v, w\}$ in the edge set $E$ of $G$. The goal of this section is to give a proof of Theorem 1.2, which we now recall.

Theorem 1.2. Let $G=(V, E)$ be a connected simple graph that contains at least one cycle of even length. Use the abbreviated notation $K:=K($ line $G)$, and let $p$ be a prime for which the quantity $k(p) \geq 1$.

Then for $G$ bipartite, one has

$$
K / p^{k(p)} K \cong \mathbb{Z}_{p^{k(p)}}^{\beta(G)-1} \oplus \mathbb{Z}_{\operatorname{gcd}\left(p^{k},|V|\right)},
$$

while for $G$ nonbipartite, one has

$$
K / p^{k(p)} K \cong \mathbb{Z}_{p^{k(p)}}^{\beta(G)-2} \oplus \begin{cases}0 & \text { if } p \text { is odd, } \\ \mathbb{Z}_{2}^{2} & \text { if } p=2 \text { and }|V| \text { is even }, \\ \mathbb{Z}_{4} & \text { if } p=2 \text { and }|V| \text { is odd. }\end{cases}
$$

Proof. One works again with the presentation from Proposition 3.1

$$
K:=K(\text { line } G)=\mathbb{Z}^{E} /\left(\mathbb{Z} e_{0}+\mathbb{Z}\left(\partial_{\text {line } G} b_{\text {line } G}(e)\right)_{e \in E}\right)
$$

for some choice of an edge $e_{0}$ in $E$. Given a vertex $v$ in $V$, let $\delta_{G}(v)$ denote the element of $\mathbb{Z}^{E}$ which is the sum with coefficient +1 of the basis elements in $\mathbb{Z}^{E}$ corresponding to edges incident with $v$. Given any edge $e=\{v, w\}$ in $E$, reasoning as in equation (12), one finds that

$$
\partial_{\text {line } G} b_{\text {line } G}(e)=\delta_{G}(v)+\delta_{G}(w)-\left(\operatorname{deg}_{G}(v)+\operatorname{deg}_{G}(w)\right) e .
$$

Letting $q:=p^{k(p)}$, one has therefore in $K / q K$ the relation

$$
\partial_{\text {line } G} b_{\text {line } G}(e) \equiv \delta_{G}(v)+\delta_{G}(w)
$$

and one can write a presentation for $K / q K$ as

$$
K / q K=\mathbb{Z}_{q}^{E} /\left(\mathbb{Z}_{q} e_{0}+\mathbb{Z}_{q}\left(\delta_{G}(v)+\delta_{G}(w)\right)_{e=\{v, w\} \in E}\right) .
$$

We now make a particular choice of the edge $e_{0}$ for this presentation, and exhibit a subset of $E$ having size $\beta(G)-2$ or $\beta(G)-1$ which will represent $\mathbb{Z}_{q}$-linearly independent elements in $K / q K$. Because $G$ contains an even-length (not necessarily minimal) cycle, it is possible to choose an edge $e_{0}$ in $E$ which lies on a minimal cycle, so that $E \backslash\left\{e_{0}\right\}$ still connects all of $V$, and so that $E \backslash\left\{e_{0}\right\}$ contains at least one odd cycle in the case where $G$ is nonbipartite. Now, in the bipartite case, pick $S \subset E \backslash\left\{e_{0}\right\}$ to be minimal with respect to the property that $S$ connects all of $V$. In the non-bipartite case, pick $S$ to be minimal with respect to the following three properties: first, $S$ must connect all of $V$; second, $S$ must contain a unique cycle; and third, this cycle must be of odd length. This means that when $G$ is bipartite, $S$ is a spanning tree that avoids $e_{0}$, and when $G$ is nonbipartite, $S$ is a unicyclic spanning subgraph that avoids $e_{0}$, whose unique cycle $C$ is of odd length.

We first wish to show that, in either case, the images of the elements $E \backslash S \backslash\left\{e_{0}\right\}$ are $\mathbb{Z}_{q^{-}}$ linearly independent in the presentation (16); note that this set $E \backslash S \backslash\left\{e_{0}\right\}$ has cardinality $\beta(G)-1$ when $G$ is bipartite, and cardinality $\beta(G)-2$ when $G$ is nonbipartite.

So assume that $E \backslash S \backslash\left\{e_{0}\right\}$ are $\mathbb{Z}_{q^{-}}$-linearly dependent in $K / q K$. Grouping the $\mathbb{Z}_{q^{-}}$ coefficients $c_{v}$ in front of each $\delta_{G}(v)$, one would have a sum $\sum_{v \in V} c_{v} \delta_{G}(v)$ lying in $\mathbb{Z}_{q} e_{0}+$ $\mathbb{Z}_{q}\left(E \backslash S \backslash\left\{e_{0}\right\}\right)$. Thus this sum should have zero coefficient on every edge $e=\{v, w\}$ in $S$, implying that $c_{v}=-c_{w}$ for every such edge. Because $S$ is a spanning set of edges, this 
forces the existence of a constant $c$ in $\mathbb{Z}_{q}$ for which every $v$ in $V$ has $c_{v}= \pm c$. In fact, when $G$ is bipartite with vertex bipartition $V=V_{1} \sqcup V_{2}$, this forces $c_{v_{1}}=c=-c_{v_{2}}$ for all $v_{1}$ in $V_{1}$ and $v_{2}$ in $V_{2}$, while for $G$ nonbipartite, the existence of the odd cycle $C$ inside $S$ forces $c_{v}=c=-c$ for all $v$ in $V$. In either case, this means that $c_{v}=-c_{w}$ for all edges $e=\{v, w\}$ in $E$, and hence the sum $\sum_{v \in V} c_{v} \delta_{G}(v)$ is actually zero in $\mathbb{Z}_{q}^{E}$. Thus the linear independence is trivial.

It only remains now to analyze the quotient

$$
\frac{K / q K}{\mathbb{Z}_{q}\left(E \backslash S \backslash\left\{e_{0}\right\}\right)}=\mathbb{Z}_{q}^{E} /\left(\mathbb{Z}_{q}(E \backslash S)+\mathbb{Z}_{q}\left(\delta_{G}(v)+\delta_{G}(w)\right)_{e=\{v, w\} \in E}\right) .
$$

Note that when $m$ is odd, for any sequence of vertices $v_{0}, v_{1}, \ldots, v_{m-1}, v_{m}$ one has a telescoping alternating sum

$$
\sum_{i=0}^{m-1}(-1)^{i}\left(\delta_{G}\left(v_{i}\right)+\delta_{G}\left(v_{i+1}\right)\right)=\delta_{G}\left(v_{0}\right)+\delta_{G}\left(v_{m}\right) .
$$

Also note that $S$ will contain paths of edges of odd length between

- every pair $\left(v_{1}, v_{2}\right)$ in $V_{1} \times V_{2}$ when $G$ is bipartite, and

- every ordered pair $(v, w)$ in $V \times V$ when $G$ is nonbipartite.

Thus, in either case, one has

$$
\mathbb{Z}_{q}\left(\delta_{G}(v)+\delta_{G}(w)\right)_{e=\{v, w\} \in E}=\mathbb{Z}_{q}\left(\delta_{G}(v)+\delta_{G}(w)\right)_{e=\{v, w\} \in S} .
$$

Using this last equation, one can rewrite the quotient on the right of (17) as

$$
\mathbb{Z}_{q}^{S} / \mathbb{Z}_{q}\left(\delta_{S}(v)+\delta_{S}(w)\right)_{e=\{v, w\} \in S}
$$

where here we regard $S$ itself as a graph, namely the edge-induced subgraph of $G=(V, E)$ having the same vertex set $V$ and edge set $S \subset E$.

Note that this last expression in (18) does not depend upon the ambient graph $G$, but only on the subgraph $S$. We therefore rename it $K_{q}(S)$ to emphasize this dependence on $S$ alone. It remains to analyze this group $K_{q}(S)$ in both the bipartite and nonbipartite cases. Case 1: $G$ is bipartite (and hence so is $S$ ). It follows that $S$ is a spanning tree on $V$, with vertex bipartition $V=V_{1} \sqcup V_{2}$. By the above discussion,

$$
\begin{aligned}
K_{q}(S) & =\mathbb{Z}_{q}^{S} / \mathbb{Z}_{q}\left(\delta_{S}(v)+\delta_{S}(w)\right)_{\left(v_{1}, v_{2}\right) \in V_{1} \times V_{2}} \\
& =\mathbb{Z}_{q}^{S} / \mathbb{Z}_{q}\left(\sum_{v \in V} c_{v} \delta_{S}(v): \sum_{v_{1} \in V_{1}} c_{v_{1}}=\sum_{v_{2} \in V_{2}} c_{v_{2}} \text { in } \mathbb{Z}_{q}\right) .
\end{aligned}
$$

We first show by induction on $|S|$ that $K_{q}(S)$ is cyclic, generated by the image of any leaf edge $e$ of $S$, that is, an edge $e$ incident to some leaf vertex $v$ having $\operatorname{deg}_{S}(v)=1$. The base case $|S|=1$ is trivial. In the inductive step, pick another leaf edge $e^{\prime}$ in $S$; we will show it has image 0 in the quotient $K_{q}(S) / \mathbb{Z}_{q} e$. If $e^{\prime}$ is incident to leaf vertex $v^{\prime}$, then for any $c$ in $\mathbb{Z}_{q}$, one has

$$
e^{\prime}+c e=\delta_{S}\left(v^{\prime}\right)+c \delta_{S}(v) .
$$

Taking $c=-1$ (respectively +1 ) when $v, v^{\prime}$ lie in the same (resp. different) set $V_{1}$ or $V_{2}$, one obtains an element that is zero in $K_{q}(S)$, and hence $e^{\prime} \equiv 0$ in $K_{q}(S) / \mathbb{Z}_{q} e$. Now, replacing $S$ by $S \backslash\left\{e^{\prime}\right\}$, one can induct on $|S|$, completing the inductive step and showing that $K_{q}(S)$ is generated by $e$.

We next analyze the order of this cyclic generator $e$ within $K_{q}(S)$. We claim that $c \cdot e=0$ in $K_{q}(S)$ if and only if $c$ lies in $|V| \mathbb{Z}_{q}$. This would finish the proof in the bipartite case, 
as it would show that $K_{q}(S)$ is isomorphic to the subgroup of $\mathbb{Z}_{q}$ generated by the element $|V|$. This subgroup is isomorphic to $\mathbb{Z}_{\operatorname{gcd}(q,|V|)}$, where $q=p^{k}$. Hence this would imply $K / q K \cong \mathbb{Z}_{p^{k(p)}}^{\beta(G)-1} \oplus \mathbb{Z}_{\mathrm{gcd}\left(p^{k(p)},|V|\right)}$, as desired.

To see the claim, assume that $c \cdot e=0$ in $K_{q}(S)$ for some $c$ in $\mathbb{Z}_{q}$. This means one has a $\operatorname{sum} \sum_{v \in V} c_{v} \delta_{G}(v)=c \cdot e$ in which $\sum_{v_{1} \in V_{1}} c_{v_{1}}=\sum_{v_{2} \in V_{2}} c_{v_{2}}$. This happens if and only if the sum has zero coefficient on all edges $e^{\prime}$ in $S \backslash\{e\}$. If $e=\{v, w\}$ with the leaf vertex $v$ lying in $V_{1}$, and $w$ in $V_{2}$, this means $c_{v_{2}}=c_{w}=-c_{v_{1}}$ for all $v_{1} \in V_{1} \backslash\{v\}$ and $v_{2} \in V_{2} \backslash\{w\}$. Then the condition $\sum_{v_{1} \in V_{1}} c_{v_{1}}=\sum_{v_{2} \in V_{2}} c_{v_{2}}$ forces

$$
c_{v}+\left(\left|V_{1}\right|-1\right)\left(-c_{w}\right)=\left|V_{2}\right|\left(c_{w}\right)
$$

i.e., $c_{v}=(|V|-1) c_{w}$. Hence this can occur if and only if $c=c_{v}+c_{w}=|V| c_{w}$, that is, if $c$ lies in $|V| \mathbb{Z}_{q}$.

Case 2. $G$ is nonbipartite (and hence so is $S$ ). In this case $S$ is a spanning unicyclic graph, whose unique cycle $C$ is of odd length. By the above discussion,

$$
\begin{aligned}
K_{q}(S) & =\mathbb{Z}_{q}^{S} / \mathbb{Z}_{q}\left(\delta_{S}(v)+\delta_{S}(w)\right)_{(v, w) \in V \times V} \\
& =\mathbb{Z}_{q}^{S} /\left\{\sum_{v \in V} c_{v} \delta_{S}(v): \sum_{v \in V} c_{v} \in 2 \mathbb{Z}_{q}\right\} .
\end{aligned}
$$

Thus, if one defines the tower of $\mathbb{Z}$-lattices (i.e., free abelian groups)

$$
L:=\mathbb{Z}^{S} \supset M:=\mathbb{Z}\left(\delta_{S}(v)\right)_{v \in V} \supset N:=\left\{\sum_{v \in V} c_{v} \delta_{S}(v): \sum_{v \in V} c_{v} \in 2 \mathbb{Z}\right\},
$$

then one has a short exact sequence

$$
0 \rightarrow \underbrace{\frac{M \otimes_{\mathbb{Z}} \mathbb{Z}_{q}}{N \otimes_{\mathbb{Z}} \mathbb{Z}_{q}}}_{(M / N) \otimes_{\mathbb{Z}} \mathbb{Z}_{q}} \rightarrow \underbrace{\frac{L \otimes_{\mathbb{Z}} \mathbb{Z}_{q}}{N \otimes_{\mathbb{Z}} \mathbb{Z}_{q}}}_{K_{q}(S)} \rightarrow \underbrace{\frac{L \otimes_{\mathbb{Z}} \mathbb{Z}_{q}}{M \otimes_{\mathbb{Z}} \mathbb{Z}_{q}}}_{(L / M) \otimes_{\mathbb{Z}} \mathbb{Z}_{q}} \rightarrow 0
$$

Here we have used on the two ends of the sequence the fact ${ }^{3}$ that for any pair of nested abelian groups $B \subset A$, one has

$$
\left(A \otimes_{\mathbb{Z}} \mathbb{Z}_{q}\right) /\left(B \otimes_{\mathbb{Z}} \mathbb{Z}_{q}\right) \cong(A / B) \otimes_{\mathbb{Z}} \mathbb{Z}_{q}
$$

Furthermore, it is easy to see that both $M / N$ and $L / M$ are isomorphic to $\mathbb{Z}_{2}$ :

- The isomorphism $M / N \cong \mathbb{Z}_{2}$ comes from choosing any $\mathbb{Z}$-basis for the lattice $M$, thus identifying $M \cong \mathbb{Z}^{|V|}$, and noting that under this identification, $N$ is identified with the index 2 sublattice $\left\{x \in \mathbb{Z}^{|V|}: \sum_{v \in V} x_{v} \in 2 \mathbb{Z}\right\}$.

- The isomorphism $L / M \cong \mathbb{Z}_{2}$ is equivalent to the assertion that the square (unsigned) edge-node incidence matrix having columns indexed by the nodes $V$ and rows indexed by the edges $S$ will have determinant \pm 2 . This is a well-known fact for connected unicyclic graphs $S$ whose unique cycle $C$ is odd; see, e.g., [17, p. 560, proof of Thm. 3.3]. It is easily proven by first checking that the determinant is scaled by \pm 1 when one removes a row and column corresponding to a leaf edge and

\footnotetext{
${ }^{3}$ That is, taking tensor products $(-)_{\otimes \mathbb{Z}} \mathbb{Z}_{q}$ is right exact, so when applied to the exact sequence
}

$$
B \rightarrow A \rightarrow A / B \rightarrow 0
$$

it gives the exact sequence

$$
B \otimes_{\mathbb{Z}} \mathbb{Z}_{q} \rightarrow A \otimes_{\mathbb{Z}} \mathbb{Z}_{q} \rightarrow(A / B) \otimes_{\mathbb{Z}} \mathbb{Z}_{q} \rightarrow 0
$$


its incident leaf vertex in $S$. This reduces the assertion to the case where $S=C$ is just an odd cycle itself, where the determinant can be calculated directly via Laplace expansion.

Hence both of the outer terms $(M / N) \otimes_{\mathbb{Z}} \mathbb{Z}_{q},(L / M) \otimes_{\mathbb{Z}} \mathbb{Z}_{q}$ in the short exact sequence (19) are isomorphic to $\mathbb{Z}_{2} \otimes_{\mathbb{Z}} \mathbb{Z}_{q}$, which vanishes for $p$ odd and equals $\mathbb{Z}_{2}$ for $p=2$. Thus (19) shows that $K_{q}(S)$ vanishes for $p$ odd, and shows for $p=2$ that $K_{q}(S)$ is either $\mathbb{Z}_{2}^{2}$ or $\mathbb{Z}_{4}$. To distinguish these possibilities when $p=2$, we analyze the additive orders of each edge $e$ in $S$ as elements of $K_{q}(S)$.

Note that for any leaf edge $e$ in $S$, say with leaf vertex $v$, one has $e=\delta_{S}(v)$, and hence $2 e=\delta_{S}(v) \equiv 0$ in $K_{q}(S)$. Thus using a leaf-induction, one sees that any edge $e$ in $S \backslash C$ has $2 e \equiv 0$ in $K_{q}(S)$.

Meanwhile, we claim that for any edge $e=\{v, w\}$ in $C$, one has $c \cdot e \equiv 0$ in $K_{q}(S)$ if and only if $|V| \cdot c$ lies in $4 \mathbb{Z}_{q}$. To see the claim, assume that $c \cdot e=0$ in $K_{q}(S)$ for some $c$ in $\mathbb{Z}_{q}$. This means one has a sum $\sum_{v \in V} c_{v} \delta_{G}(v)=c \cdot e$ with $\sum_{v \in V} c_{v} \in 2 \mathbb{Z}_{q}$. This happens if and only if the sum has zero coefficient on all edges $e^{\prime}$ in $S \backslash\{e\}$. Applying this for the edges $e^{\prime}$ in $C \backslash\{e\}$, one concludes that $c_{v}=c_{w}$, and hence $c=c_{v}+c_{w}=2 c_{v}$. Applying this for the remaining edges $e^{\prime}$ in $S \backslash C$, one concludes that $c_{w}= \pm c_{v}$ for all $w$ in $V$. But then the condition that $\sum_{w \in V} c_{w}$ lies in $2 \mathbb{Z}_{q}$ means that $|V| \cdot c_{v}$ also lies in $2 \mathbb{Z}_{q}$, i.e., that $|V| \cdot c=2|V| \cdot c_{v}$ lies in $4 \mathbb{Z}_{q}$. One concludes that edges $e$ in $C$ have order 2 when $|V|$ is even, and order 4 when $|V|$ is odd. Since every edge $e$ in $S \backslash C$ has $2 e \equiv 0$ in $K_{q}(S)$, this implies $K_{q}(S) \cong \mathbb{Z}_{2}^{2}$ when $|V|$ is even and $K_{q}(S) \cong \mathbb{Z}_{4}$ when $|V|$ is odd.

\section{Proof of Theorem 1.3}

Recall here the statement of Theorem 1.3.

Theorem 1.3. For any connected d-regular simple graph $G$ with $d \geq 3$, there is a group homomorphism

$$
K(\text { line } G) \stackrel{f}{\rightarrow} K(\operatorname{sd} G)
$$

whose kernel-cokernel exact sequence takes the form

$$
0 \rightarrow \mathbb{Z}_{d}^{\beta(G)-2} \oplus C \rightarrow K(\text { line } G) \stackrel{f}{\rightarrow} K(\operatorname{sd} G) \rightarrow C \rightarrow 0
$$

in which the cokernel $C$ is the following cyclic d-torsion group:

$$
C= \begin{cases}0 & \text { if } G \text { is non-bipartite and } d \text { is odd, } \\ \mathbb{Z}_{2} & \text { if } G \text { is non-bipartite and } d \text { is even, } \\ \mathbb{Z}_{d} & \text { if } G \text { is bipartite. }\end{cases}
$$

6.1. Defining the morphism $f$. We begin our proof of the theorem by first defining a linear map $f: \mathbb{R}^{E_{\text {line } G}} \rightarrow \mathbb{R}^{E_{\text {sd } G}}$ which will turn out to be a morphism of rational orthogonal decompostions.

Definition 6.1. Define a $\mathbb{R}$-linear map $f: \mathbb{R}^{E_{\text {line } G} \rightarrow \mathbb{R}^{E_{\mathrm{sd} G}} \text { by setting }}$

$$
f(u v, v w)=(u v, v)+(v, v w)
$$

for every pair of edges $\{u, v\},\{v, w\}$ of $G$ incident at some vertex $v$ (see Figure 4). Equivalently, the adjoint map $f^{t}$ is defined by

$$
f^{t}(u v, v)=\sum_{w \in V:\{v, w\} \in E}(u v, v w) .
$$



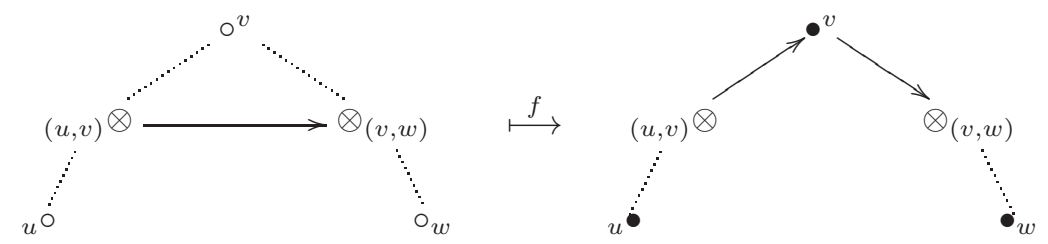

Figure 4. The action of $f$ on a single edge of line $G$.

The following definitions and lemma will be useful both for showing that $f$ gives a morphism, and in our later analysis.

Definition 6.2. Given a directed cycle

$$
C=\left\{\left(v_{1}, v_{2}\right),\left(v_{2}, v_{3}\right), \ldots,\left(v_{m-1}, v_{m}\right),\left(v_{m}, v_{1}\right)\right\}
$$

in $G$, let

$$
\begin{aligned}
\operatorname{sd} C & :=\left\{\left(v_{1}, v_{1} v_{2}\right),\left(v_{1} v_{2}, v_{2}\right),\left(v_{2}, v_{2} v_{3}\right),\left(v_{2} v_{3}, v_{3}\right), \ldots\right\} \\
\text { line } C & :=\left\{\left(v_{1} v_{2}, v_{2} v_{3}\right),\left(v_{2} v_{3}, v_{3} v_{4}\right), \ldots,\left(v_{m-1} v_{m}, v_{m} v_{1}\right),\left(v_{m} v_{1}, v_{1} v_{2}\right)\right\}
\end{aligned}
$$

denote corresponding cycles in sd $G$, line $G$.

Cycles in line $G$ of the form line $C$ where $C$ is a cycle of $G$ will be called global cycles. A cycle in line $G$ will be called local (to vertex $v$ ) if every vertex $v_{i} v_{j}$ of line $G$ visited by the cycle has $v \in\left\{v_{i}, v_{j}\right\}$.

Lemma 6.3. Let $G$ be a graph, and let $\{C\}$ be a set of directed cycles indexing a spanning set $\{z(C)\}$ for the cycle space $Z_{G}$. Then

(1) $Z_{\mathrm{sd} G}$ will be spanned by the incidence vectors $\{z(\operatorname{sd} C)\}$ of the associated subdivided cycles, and

(2) $Z_{\text {line } G}$ will be spanned by the incidence vectors $\{z$ (line $\left.C)\right\}$ for their associated global cycles together with all local cycles.

Proof. Assertion (1) of was implicitly used in Section 3.3, and should be clear either from elementary algebraic topology or from the discussion of bases for $Z_{G}$ coming from spanning forests at the beginning of Section 3.1.

For assertion (2), given any directed cycle in line $G$, put an equivalence relation on its edges by taking the transitive closure of the following relation: two consecutive edges $(u v, v w),(v w, w x)$ in the cycle are equivalent if there exists a vertex $y$ of $G$ contained in $\{u, v\} \cap\{v, w\} \cap\{w, x\}$. The global cycles in line $G$ are by definition those in which the equivalence classes for this relation all have cardinality two (N.B.: here one is using the assumption that $G$ is simple). Given a cycle $z$ in line $G$ that contains equivalence classes of size at least 3 , it is easy to see that one can always add a local cycle to $z$ and reduce the number of such equivalence classes: if the equivalence class and its neighbors in $z$ correspond to these terms

$$
\cdots+\left(a b_{1}, y b_{1}\right)+\left(y b_{1}, y b_{2}\right)+\left(y b_{2}, y b_{3}\right)+\cdots+\left(y b_{t-1}, y b_{t}\right)+\left(y b_{t}, b_{t} c\right)+\cdots
$$

where $a, c \neq y$, then subtracting the local cycle

$$
\left(y b_{1}, y b_{2}\right)+\left(y b_{2}, y b_{3}\right)+\cdots+\left(y b_{t-1}, y b_{t}\right)+\left(y b_{t}, y b_{1}\right)
$$

gives a result that looks locally like

$$
\cdots+\left(a b_{1}, y b_{1}\right)+\left(y b_{1}, y b_{t}\right)+\left(y b_{t}, b_{t} c\right)+\cdots .
$$



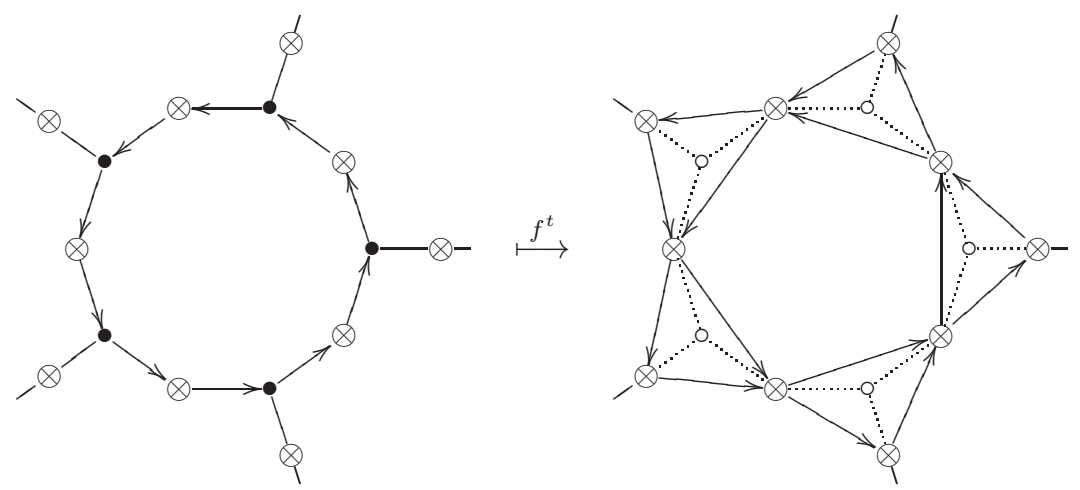

Figure 5. An example of a subdivided cycle in sd $G$, and its image under $f^{t}$ in line $G$ when $G$ is 3-regular.

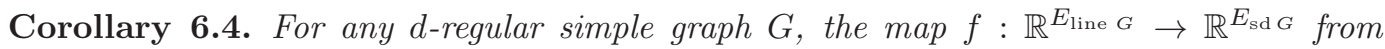
Definition 6.1 is a morphism of the associated rational orthogonal decompositions, and hence induces a group homomorphism

$$
f: K(\text { line } G) \rightarrow K(\operatorname{sd} G) .
$$

Proof. By Proposition 2.3, one must show both $f\left(Z_{\text {line } G}\right) \subset Z_{\text {sd } G}$ and $f^{t}\left(Z_{\text {sd } G}\right) \subset Z_{\text {line } G}$.

To show $f\left(Z_{\text {line } G}\right) \subset Z_{\text {sd } G}$, using Lemma 6.3 (ii), it suffices to show that $f$ takes both global and local cycles in line $G$ to cycles in $Z_{\mathrm{sd} G}$. This is easy (and requires no assumption about the $d$-regularity of $G$ ): local cycles map to 0 under $f$, and a global cycle of the form line $C$ satisfies $f(z($ line $C))=z(\operatorname{sd} C)$.

To show $f^{t}\left(Z_{\mathrm{sd} G}\right) \subset Z_{\text {line } G}$, using Lemma $6.3(\mathrm{i})$, it suffices to show for every directed cycle $C$ in $G$ that $f$ takes the subdivided cycle

$$
z(\operatorname{sd} C)=\left(v_{1} v_{2}, v_{2}\right)+\left(v_{2}, v_{2} v_{3}\right)+\left(v_{2} v_{3}, v_{3}\right)+\left(v_{3}, v_{3} v_{4}\right)+\cdots+\left(v_{k} v_{1}, v_{1}\right)+\left(v_{1}, v_{1} v_{2}\right)
$$

to a sum of cycles in $Z_{\text {line } G}$. The regularity of $G$ implies that each $v_{i}$ has $d-2$ neighbors off the cycle; label them $u_{i}^{1}, \ldots, u_{i}^{d-2}$. Then one can write

$$
\left(f^{t}\right)(z(\operatorname{sd} C))=2 z(\text { line } C)+\zeta_{1}+\cdots+\zeta_{d-2}
$$

where for $j=1,2, \ldots, d-2$ one defines the element of $Z_{\text {line } G}$

$$
\begin{aligned}
\zeta_{j}:=\left(v_{1} v_{2}, v_{2} u_{2}^{j}\right)+\left(v_{2} u_{2}^{j}, v_{2} v_{3}\right)+\left(v_{2} v_{3}, v_{3} u_{3}^{j}\right) & +\left(v_{3} u_{3}^{j}, v_{3} v_{4}\right)+\cdots \\
& +\left(v_{k} v_{1}, v_{1} u_{1}^{j}\right)+\left(v_{1} u_{1}^{j}, v_{1} v_{2}\right) .
\end{aligned}
$$

An example with $d=3$ is shown in Figure 5, depicting the subdivided cycle $\operatorname{sd} C$ in $\operatorname{sd} G$, and then its image under $f^{t}$ in line $G$, which decomposes into 2 copies of the inner cycle line $C$ along with $1(=d-2)$ outer cycle $\zeta_{1}$.

\subsection{The kernel and cokernel of $f$ are $d$-torsion.}

Proposition 6.5. For any d-regular connected graph $G$, both maps

$$
\begin{aligned}
& f^{t} f: K(\text { line } G) \rightarrow K(\text { line } G) \\
& f f^{t}: K(\operatorname{sd} G) \rightarrow K(\operatorname{sd} G)
\end{aligned}
$$

are scalar multiplications by $d$. 
Proof. The proofs of these are straightforward computations:

$$
\begin{aligned}
f^{t} f(u v, v w) & =f^{t}(u v, v)+f^{t}(v, v w) \\
& =\sum_{x \in V:\{v, x\} \in E}(u v, v x)+(x v, v w) \\
& =d \cdot(u v, v w)+\sum_{x \in V:\{v, x\} \in E}((u v, v x)+(x v, v w)+(v w, u v)) \\
& =d \cdot(u v, v w) \bmod Z_{\text {line } G .} \\
f f^{t}(u v, v) & =\sum_{x \in V:\{v, x\} \in E} f(u v, v x) \\
& =\sum_{x \in V:\{v, x\} \in E}(u v, v)+(v, v x) \\
& =d \cdot(u v, v)+\sum_{x \in V:\{v, x\} \in E}(v, v x) \\
& =d \cdot(u v, v) \bmod B_{\mathrm{sd} G} .
\end{aligned}
$$

Corollary 6.6. For any d-regular connected graph $G$, both $\operatorname{ker}(f)$ and $\operatorname{coker}(f)$ are all d-torsion.

Proof. For $x \in \operatorname{ker}(f)$ and $y \in \operatorname{coker}(f)$, one has

$$
\begin{aligned}
& d \cdot x=f^{t} f(x)=f^{t}(0)=0, \\
& d \cdot y=f f^{t}(y) \in \operatorname{im}(f) .
\end{aligned}
$$

\subsection{Analyzing the cokernel.}

Proposition 6.7. For any d-regular connected graph $G$, the group $C:=\operatorname{coker}(f)$ is a cyclic group as described in Theorem 1.3.

Proof. We will use the presentation

$$
C:=\operatorname{coker}(f):=K(\operatorname{sd} G) / \operatorname{im}(f)=\mathbb{Z}^{E_{\mathrm{sd} G}} /\left(B_{\mathrm{sd} G}+Z_{\mathrm{sd} G}+\operatorname{im}(f)\right) .
$$

To see that $C$ is cyclic, note that there are two ways for a pair of edges in $\operatorname{sd} G$ to be incident at a vertex, and in either case their images in $C$ will differ by a sign:

$$
\begin{aligned}
& (u, u v)=-(u v, v) \quad \bmod B_{\mathrm{sd} G}, \\
& (u v, v)=+(v, v w) \quad \bmod \operatorname{im}(f) .
\end{aligned}
$$

Since $G$ is connected, this shows $C$ is cyclic, generated by the image of any directed edge of sd $G$. Furthermore, it is a quotient of $\mathbb{Z}_{d}$ by Corollary 6.6.

When $G$ is bipartite, in order to show $C=\mathbb{Z}_{d}$, it will suffice to exhibit a surjection $C \rightarrow \mathbb{Z}_{d}$. Let the vertex set $V$ for $G$ have bipartition $V=V_{1} \sqcup V_{2}$, and consider the abelian group homomorphism $\phi: \mathbb{Z}^{E_{\text {sd }} G} \rightarrow \mathbb{Z}$ which maps a typical directed edge $\left(v_{1}, v_{1} v_{2}\right)$ or $\left(v_{1} v_{2}, v_{2}\right)$ in $\operatorname{sd} G$ (where $v_{i} \in V_{i}$ for $\left.=1,2\right)$ to $1 \in \mathbb{Z}$. One can check that each of the three subgroups $B_{\mathrm{sd} G}, Z_{\mathrm{sd} G}, \operatorname{im}(f)$ by which one mods out in (20) is mapped via $\phi$ into the subgroup $d \mathbb{Z}$ :

- Any directed cycle $C$ in $\operatorname{sd} G$ has $\phi(z(C))=0$ (due to the fact that $C$ will have even length),

- any edge $e$ of line $G$ has $\phi(f(e))=0$,

- any vertex $v_{1} v_{2}$ in $\operatorname{sd} G$ has $\phi\left(b_{\mathrm{sd} G}\left(v_{1} v_{2}\right)\right)=0$, while 
- any vertex $v_{i}$ in $\operatorname{sd} G$ has $\phi\left(b_{\text {sd } G}\left(v_{i}\right)\right)=d$.

Thus $\phi$ induces a surjection from $C$ onto $\mathbb{Z}_{d}$, as desired.

If $G$ is not bipartite, it contains some (directed) odd cycle $C$. Pick any directed edge $e$ in the subdivision sd $C$ and use the two relations (a), (b) to rewrite it successively as \pm the other directed edges in the cycle. It changes sign each time one uses (a) to pass through a vertex of sd $C$ that comes from an edge of $G$. Since there are an odd number of such edges in the cycle, it will change sign an odd number of times before it returns, yielding

$$
e=-e \bmod B_{\mathrm{sd} G}+\operatorname{im}(f) .
$$

Hence $2 e=0$ in $C$, so $C$ is a quotient of $\mathbb{Z}_{2}$.

Since $C$ is also a quotient of $\mathbb{Z}_{d}$, when $d$ is odd, one must have $C=0$. When $d$ is even, consider the index 2 sublattice $\Lambda$ of $\mathbb{Z}^{E_{\text {sd }}}$ consisting of those vectors whose sum of coordinates is even. Without any parity assumption on $d$, it is true that $\operatorname{im}(f) \subset \Lambda$ (by definition of $f$ ) and $Z_{\operatorname{sd} G} \subset \Lambda$ (because the subdivided cycles sd $C$ have evenly many edges). The assumption that $d$ is even implies that $B_{\mathrm{sd} G}$ also lies in $\Lambda: B_{\mathrm{sd} G}$ is generated by the bonds in $\operatorname{sd} G$ of the form $b_{\text {sd } G}(v)$ for vertices $v$ of sd $G$, and every vertex in $\operatorname{sd} G$ has degree either 2 or $d$. Consequently, the presentation (20) shows that $C$ surjects onto $\mathbb{Z}^{E_{\text {sd } g} / \Lambda \cong \mathbb{Z}_{2}}$

6.4. Analyzing the kernel. It remains to understand $\operatorname{ker}(f)$, or equivalently by Proposition 2.6, to understand its Pontrjagin dual

$$
\operatorname{coker}\left(f^{t}\right)=\mathbb{Z}^{E_{\text {line } G}} /\left(Z_{\text {line } G}+B_{\text {line } G}+\operatorname{im}\left(f^{t}\right)\right) .
$$

This will come about by reformulating this presentation, in order to analyze it locally.

Definition 6.8. For each vertex $v \in V_{G}$ of a $d$-regular simple graph $G=\left(V_{G}, E_{G}\right)$, define inside line $G$ the $d$-clique local to $v$

$$
K_{d}^{(v)}=\left(V\left(K_{d}^{(v)}\right), E\left(K_{d}^{(v)}\right)\right)
$$

to be the vertex-induced subgraph of line $G$ on the vertex set

$$
V\left(K_{d}^{(v)}\right):=\left\{v w: v w \in E_{G}\right\} .
$$

Note that the edges of line $G$ form a disjoint decomposition

$$
E_{\text {line } G}=\bigsqcup_{v \in V_{G}} E\left(K_{d}^{(v)}\right)
$$

since $G$ was assumed to be a simple graph. Also note that a cycle in line $G$ is local to vertex $v$, as in Definition 6.2, if and only if it is supported on the edges $E\left(K_{d}^{(v)}\right)$. If one lets $Z_{\text {line } G}^{\text {global }}$ be the span of global cycles $\left\{z_{\text {line }} C\right\}$ coming from any spanning set of cycles $\left\{z_{C}\right\}$ for $Z_{G}$, then Lemma 6.3 (ii) implies

$$
Z_{\text {line } G}=Z_{\text {line } G}^{\text {local }}+Z_{\text {line } G}^{\text {global }} .
$$

To simplify the presentation (21), note that for a vertex $v w$ of line $G$, the bond

$$
b_{\text {line } G}(v w)=f^{t}(v w, v)+f^{t}(v w, w)
$$


lies in $\operatorname{im}\left(f^{t}\right)$, and consequently, $B_{\text {line } G} \subset \operatorname{im}\left(f^{t}\right)$. Note also that the decomposition (22) leads to a family of compatible direct sum decompositions

$$
\begin{aligned}
\mathbb{Z}^{E_{\text {line } G}} & =\bigoplus_{v \in V_{G}} \mathbb{Z}^{E\left(K_{d}^{(v)}\right)} \\
Z_{\text {line } G}^{\text {local }} & =\bigoplus_{v \in V_{G}} Z_{K_{d}^{(v)}} \\
\operatorname{im}\left(f^{t}\right) & =\bigoplus_{v \in V_{G}} B_{K_{d}^{(v)}}
\end{aligned}
$$

This gives the simplified presentation

$$
\begin{aligned}
\operatorname{coker}\left(f^{t}\right) & =\left(\bigoplus_{v \in V_{G}} \mathbb{Z}^{E\left(K_{d}^{(v)}\right)} /\left(B_{K_{d}^{(v)}}+Z_{K_{d}^{(v)}}\right)\right) / Z_{\text {line } G}^{\text {global }} \\
& =\left(\bigoplus_{v \in V_{G}} K\left(K_{d}^{(v)}\right)\right) / Z_{\text {line } G}^{\text {global }}
\end{aligned}
$$

We use this presentation to prove the following lemma, which together with Proposition 6.7 immediately implies Theorem 1.3.

Lemma 6.9. For a connected $d$-regular graph $G$,

$$
\operatorname{ker}(f) \cong \mathbb{Z}_{d}^{\beta(G)-2} \oplus C
$$

where $C:=\operatorname{coker}(f)$ is as described in Theorem 1.3.

Proof. We claim that it suffices to prove these two bounds on $\operatorname{ker}(f)$ :

(i) There is a surjection $\operatorname{ker}(f) \rightarrow \mathbb{Z}_{d}^{\beta(G)-2}$ and,

(ii) $\operatorname{ker}(f)$ can be generated by $\beta(G)-1$ elements.

To see this claim, note that since $\operatorname{ker}(f)$ is all $d$-torsion by Corollary 6.6 , assertion (ii) would imply a surjection $\mathbb{Z}_{d}^{\beta(G)-1} \rightarrow \operatorname{ker}(f)$. Together with (i), this would imply $\operatorname{ker}(f) \cong$ $\mathbb{Z}_{d}^{\beta(G)-2} \oplus C^{\prime}$ for some cyclic group $C^{\prime}$. But then exactness of the sequence

$$
0 \rightarrow \underbrace{\operatorname{ker}(f)}_{\mathbb{Z}_{d}^{\beta(G)-2} \oplus C^{\prime}} \rightarrow K(\text { line } G) \stackrel{f}{\rightarrow} K(\operatorname{sd} G) \rightarrow \underbrace{\operatorname{coker}(f)}_{C} \rightarrow 0
$$

forces

$$
\left(d^{\beta(G)-2}\left|C^{\prime}\right|\right)|K(\operatorname{sd} G)|=\mid K(\text { line } G)|| C \mid .
$$

From this equation and equation (2) one deduces $\left|C^{\prime}\right|=|C|$. Since both $C^{\prime}$ and $C$ are cyclic, this means $C^{\prime} \cong C$, as desired.

In the proofs of assertions (i) and (ii), one uses the fact that $\operatorname{ker}(f)=\operatorname{coker}\left(f^{t}\right)$. Moreover, setting $n:=\left|V_{G}\right|$, one can rewrite the direct sum from (23) as

$$
\bigoplus_{v \in V_{G}} K\left(K_{d}^{(v)}\right) \cong \bigoplus_{v \in V_{G}} \mathbb{Z}_{d}^{d-2} \cong \mathbb{Z}_{d}^{n(d-2)}
$$

For assertion (i), we use some easy numerology. Note that $Z_{\text {line } G}^{\text {global }}$ can be generated by $\beta(G)$ elements, and also that

$$
\beta(G)=\left|E_{G}\right|-\left|V_{G}\right|+1=\frac{d n}{2}-n+1=\frac{n(d-2)}{2}+1
$$


so that

$$
n(d-2)-\beta(G)=\beta(G)-2 .
$$

Since it is easily seen that that any quotient of an abelian group $\mathbb{Z}_{d}^{a}$ by a subgroup that can be generated by $b$ elements will have a surjection to $\mathbb{Z}_{d}^{a-b}$, one can apply this with $a=n(d-2)$ and $b=\beta(G)$ to the presentation (23), and conclude that there is a surjection $\operatorname{coker}\left(f^{t}\right) \rightarrow \mathbb{Z}_{d}^{\beta(G)-2}$.

For assertion (ii), the idea will be to start with the

$$
n(d-2)=2(\beta(G)-1)
$$

generators in (24), and use (all but one of) the $\beta(G)$ generating global cycles in $Z_{\text {line } G}^{\text {global }}$ to rewrite them in terms of other generators, with $\beta(G)-1$ generators left. This will be achieved by removing the vertices from $G$ one at a time in a certain order, in order to control the rewriting process.

To this end, order the vertices $V_{G}$ as $v_{1}, v_{2}, \ldots, v_{n}$ in such a way that the vertex-induced subgraphs

$$
\begin{aligned}
G_{i} & :=G \backslash\left\{v_{1}, v_{2}, \ldots, v_{i-1}\right\} \\
& \left(\text { so } G_{1}:=G, \text { and } G_{n} \text { has one vertex } v_{n}\right)
\end{aligned}
$$

satisfy

$$
d_{i}:=\operatorname{deg}_{G_{i}}\left(v_{i}\right)<d \text { for } i \geq 2 .
$$

For each $i \geq 1$, partition the $d_{i}$ neighbors $v_{i}$ in $G_{i}$ into blocks $A_{1}, A_{2}, \ldots, A_{c_{i}}$ according to the connected components of $G_{i+1}$ in which they lie. The number $c_{i}$ of such components coincides with the number of connected components in $G_{i+1}$ into which the connected component of $v_{i}$ in $G_{i}$ splits after removing $v_{i}$. Define

$$
\Delta_{i}:=d_{i}-c_{i}=\beta\left(G_{i}\right)-\beta\left(G_{i+1}\right),
$$

where the last equality follows from the Euler relation for graphs:

$$
\left|V_{G}\right|-\left|E_{G}\right|=\mid\{\text { connected components of } G\}|-| \beta(G) \mid .
$$

Consequently,

$$
\Delta_{1}+\Delta_{2}+\cdots+\Delta_{n-1}=\beta\left(G_{1}\right)-\beta\left(G_{n}\right)=\beta(G) .
$$

Our goal will then be to find $\Delta_{i}$ minimal generators of (24) to remove at each stage $i \geq 2$ (and at the first stage $i=1$, remove one fewer, that is, $\Delta_{1}-1=d-2$ of them). This would leave a generating set for $\operatorname{coker}\left(f^{t}\right)$ of cardinality $n(d-2)-(\beta(G)-1)=\beta(G)-1$, as desired.

For $i \geq 2$, inside the clique $K_{d}^{\left(v_{i}\right)}$ local to $v_{i}$, choose a forest $F_{i}$ of edges having $c_{i}$ components which are spanning trees on each of the subsets $\left\{v_{i} x: x \in A_{j}\right\}$ for $j=1,2, \ldots, c_{i}$. Note that

$$
\left|F_{i}\right|=\sum_{j=1}^{c_{i}}\left(\left|A_{j}\right|-1\right)=d_{i}-c_{i}=\Delta_{i} .
$$

Also note that the forest $F_{i}$ manages to avoid touching at least one vertex in the $d$-clique $K_{d}^{\left(v_{i}\right)}$, namely any vertex of the form $v_{i} v_{k}$ in which $\left\{v_{i} v_{k}\right\} \in E_{G}$ and $k<i$; there will exist at least one such $k$ since by construction, $\operatorname{deg}_{G_{i}}\left(v_{i}\right)=d_{i}<d=\operatorname{deg}_{G}\left(v_{i}\right)$.

Hence by Proposition 3.3, the edges in $F_{i}$ give $\Delta_{i}$ generators that could be completed to a set of $d-2$ minimal generators for $K\left(K_{d}^{\left(v_{i}\right)}\right) \cong \mathbb{Z}_{d}^{d-2}$. Each of these generators in $F_{i}$ can be re-written, using a cycle in $Z_{\text {line } G}^{\text {global }}$, in terms of generators from $K\left(K^{\left(v_{k}\right)}\right)$ 's that have $k>i$, as follows. Given any edge $\left(v_{i} x, v_{i} x^{\prime}\right)$ in $F_{i}$, there is a path from $x$ to $x^{\prime}$ in $G_{i+1}$ (because $x, x^{\prime}$ lie in the same component of $G_{i+1}$ by construction), and hence a directed 


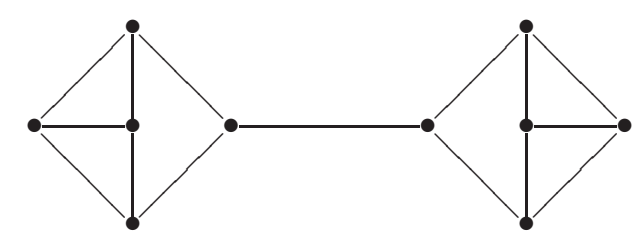

FiguRE 6. A 3-regular connected graph which is not 2-edge-connected.

cycle $C$ in $G_{i}$ going from $v_{i}$ to $x$ then through this path to $x^{\prime}$ and back to $v_{i}$. The global cycle $z$ (line $C$ ) allows one to rewrite $\left(v_{i} x, v_{i} x^{\prime}\right)$ as desired.

The only difference for $i=1$ is that, even when $\Delta_{1}=d-1$ (that is, when $c_{1}=1$ ), in this situation choose $F_{i}$ to have at most $d-2$ edges (that is, remove any edge from the forest $F_{1}$ if $\left.c_{1}=1\right)$. This modification ensures that one can still apply Proposition 3.3 and rewrite all of the generators of $K\left(K_{d}^{\left(v_{1}\right)}\right)$ corresponding to the edges of $F_{1}$.

Remark 6.10. One should remark that for a connected, $d$-regular graph $G$, the extra hypothesis in Theorem 1.3 that $G$ is 2-edge-connected is well-known (see, e.g., [9]) to be superfluous when $d$ is even: a connected graph $G$ with all vertices of even degree cannot have a cut-edge, as the two components created by the removal of this edge would each be graphs having exactly one vertex of odd degree, an impossibility.

However, when $d$ is odd, the extra hypothesis of 2-edge-connectivity need not follow. For example, the 3-regular graph shown in Figure 6 is connected, but not 2-edge-connected.

\section{Proof of Corollary 1.4}

In this section we prove Corollary 1.4. Informally, the corollary states that critical group of $G$ determines the critical group of line $G$ in a simple way.

Corollary 1.4. For $G$ a simple, connected, $d$-regular graph with $d \geq 3$ which is nonbipartite, after expressing uniquely

$$
K(G) \cong \bigoplus_{i=1}^{\beta(G)} \mathbb{Z}_{d_{i}}
$$

with $d_{i}$ dividing $d_{i+1}$, one has

$$
K(\text { line } G) \cong\left(\begin{array}{ll}
\beta(G)-2 \\
\bigoplus_{i=1} \mathbb{Z}_{2 d d_{i}}
\end{array}\right) \oplus \begin{cases}\mathbb{Z}_{2 d_{\beta(G)-1}} \oplus \mathbb{Z}_{2 d_{\beta(G)}} & \text { for }|V| \text { even } \\
\mathbb{Z}_{4 d_{\beta(G)-1}} \oplus \mathbb{Z}_{d_{\beta(G)}} & \text { for }|V| \text { odd. }\end{cases}
$$

Proof. Let $K:=K($ line $G$ ), and fix a prime $p$. Our goal is to show that the $p$-primary component of $K$ matches that of the group on the right side of (25).

The hypotheses of the theorem allow one to apply the nonbipartite cases of Theorem 1.2 and Theorem 1.3. The former asserts that

$$
K / p^{k(p)} K \cong \mathbb{Z}_{p^{k(p)}}^{\beta(G)-2} \oplus \begin{cases}0 & \text { for } p \text { odd } \\ \mathbb{Z}_{2}^{2} & \text { for } p=2,|V| \text { even } \\ \mathbb{Z}_{4} & \text { for } p=2,|V| \text { odd }\end{cases}
$$

while the latter gives an exact sequence

$$
0 \rightarrow \mathbb{Z}_{d}^{\beta(G)-2} \oplus \mathbb{Z}_{\operatorname{gcd}(2, d)} \rightarrow K \rightarrow K(\operatorname{sd} G) \rightarrow \mathbb{Z}_{\operatorname{gcd}(2, d)} \rightarrow 0 .
$$


In analyzing the $p$-primary component $\operatorname{Syl}_{p}(K)$, it is convenient to define the type of a finite abelian $p$-group $A$ as the unique integer partition $\nu=\left(\nu_{1} \geq \nu_{2} \geq \cdots\right)$ for which $A \cong \bigoplus_{i \geq 1} \mathbb{Z}_{p^{\nu_{i}}}$. Let $\mu, \lambda$ denote the types of $\operatorname{Syl}_{p}(K(G)), \operatorname{Syl}_{p}(K)$, where we think of both $\mu, \lambda$ as partitions with $\beta(G)$ parts (allowing some parts to be 0 ). Note that Proposition 3.2 asserts, in this language, that $\operatorname{Syl}_{p}(K(\operatorname{sd} G))$ has type $\mu$ for $p$ odd and type $\mu+\left(1^{\beta(G)}\right)$ for $p=2$.

A basic fact from the theory of Hall polynomials [13, Chapter II Section 9] says that there exist short exact sequences of abelian $p$-groups

$$
0 \rightarrow A \rightarrow B \rightarrow C \rightarrow 0
$$

in which $A, B, C$ have types $\nu, \lambda, \mu$, respectively, if and only if the Littlewood-Richardson (or $L R$ ) coefficient $c_{\mu, \nu}^{\lambda}$ does not vanish. The combinatorial rephrasing of this $L R$-condition is as follows: there must exist at least one column-strict tableau (which we will call an $L R$ tableau) of the skew-shape $\lambda / \mu$ having content $\nu$, for which the word obtained by reading the tableau (in English notation) from right-to-left in each row, starting with the top row, is Yamanouchi. Here the Yamanouchi condition means that within each initial segment of the word, and for each value $i \geq 1$, the number of occurrences of $i+1$ is at most the number of occurrences of $i$. See [13, Chapter I $\S 9]$ and [16, Appendix $\S A 1.3]$ for more on these notions.

Suppose that $p$ is odd. Then $k(p)$ is the largest power such that $p^{k(p)}$ divides $d$, so taking the $p$-primary components in (27), we obtain the following short exact sequence:

$$
0 \longrightarrow \underbrace{\mathbb{Z}_{p^{k(p)}}^{\beta(G)-2}}_{\text {type } \nu=\left(k(p)^{\beta(G)-2}\right)} \longrightarrow \underbrace{\operatorname{Syl}_{p} K}_{\text {type } \lambda} \longrightarrow \underbrace{\operatorname{Syl}_{p}(K(\operatorname{sd} G))}_{\text {type } \mu} \longrightarrow 0
$$

where $\lambda$ has at most $\beta(G)-2$ nonzero parts by (26). Since nonvanishing of the LR-coefficient $c_{\mu, \nu}^{\lambda}$ forces $\mu \subset \lambda$, it must be that $\mu$ also has at most $\beta(G)-2$ nonzero parts. Furthermore, one can check that column-strictness together with the Yamanouchi condition on the reading word of an LR-tableau of shape $\lambda / \mu$ and content $\nu=\left(k(p)^{\beta(G)-2}\right)$ uniquely determine the tableau: it must have each entry in row $i$ equal to $i$ for $i=1,2, \ldots, \beta(G)-2$. This forces $\lambda_{i}=\mu_{i}+k(p)$ for $i=1,2, \ldots, \beta(G)-2$, and hence $\lambda$ agrees with the type of the $p$-primary component on the right side of (25).

Suppose that $p=2$, so that $2^{k(p)-1}$ divides $d$, but $2^{k(p)}$ does not.

When $d$ is odd we have that $k(p)=1$. On the other hand, taking the 2-primary components in (27) shows that $\operatorname{Syl}_{2} K \cong \operatorname{Syl}_{2}(K(\operatorname{sd} G))$, so $\lambda=\mu+\left(1^{\beta(G)}\right)$. Since $d$ is odd, $|V|$ must be even (as the $d$-regularity of $G$ forces $d|V|=2|E|$ ), so this $\lambda$ again agrees with the type of the 2-primary component on the right side of (25).

If $d$ is even, the 2-primary components in (27) form the following exact sequence

$$
0 \longrightarrow \underbrace{\mathbb{Z}_{2^{k(p)-1} \oplus \mathbb{Z}_{2}}^{\beta(G)-2}}_{\text {type } \nu=\left((k(p)-1)^{\beta(G)-2}, 1\right)} \longrightarrow \underbrace{\operatorname{Syl}_{2} K}_{\text {type } \lambda} \longrightarrow \underbrace{\operatorname{Syl}_{2}(K(\operatorname{sd} G))}_{\text {type } \mu+\left(1^{\beta(G)}\right)} \stackrel{\pi}{\rightarrow} \mathbb{Z}_{2} \longrightarrow 0 .
$$

This can be truncated to the following short exact sequence involving $\operatorname{ker} \pi$ :

$$
0 \longrightarrow \underbrace{\mathbb{Z}_{2^{k(p)-1} \oplus \mathbb{Z}_{2}}^{\beta(G)-2}}_{\text {type } \left.\nu=(k(p)-1)^{\beta(G)-2}, 1\right)} \longrightarrow \underbrace{\operatorname{Syl}_{2} K}_{\text {type } \lambda} \longrightarrow \underbrace{\operatorname{ker} \pi}_{\text {type } \hat{\mu}} \longrightarrow 0
$$

for some partition $\hat{\mu}$, and where the last two parts $\left(\lambda_{\beta(G)-1}, \lambda_{\beta(G)}\right)$ in $\lambda$ are either $(1,1)$ or $(2,0)$ by $(26)$, depending on the parity of $|V|$. 
The short exact sequence

$$
0 \rightarrow \operatorname{ker} \pi \rightarrow \operatorname{Syl}_{2}(K(\operatorname{sd} G)) \stackrel{\pi}{\rightarrow} \mathbb{Z}_{2} \rightarrow 0
$$

shows that $\hat{\mu}$ is obtained from $\mu+\left(1^{\beta(G)}\right)$ by removing one square; we claim that $\hat{\mu}$ can have at most $\beta(G)-1$ nonzero parts, and hence this square must be removed from the last row, that is, $\hat{\mu}=\mu+\left(1^{\beta(G)-1}, 0\right)$. The reason for this claim is that, since the LR-coefficient $c_{\hat{\mu}, \nu}^{\lambda} \neq 0$, the LR-condition forces

$$
\sum_{i \geq \beta(G)-1} \lambda_{i} \geq \sum_{i \geq \beta(G)-1} \hat{\mu}_{i}+\sum_{i \geq \beta(G)-1} \nu_{i} .
$$

As $\sum_{i \geq \beta(G)-1} \lambda_{i}=2$ in both cases for the parity of $|V|$, and $\sum_{i \geq \beta(G)-1} \nu_{i}=1$, this forces $\sum_{i \geq \beta(G)-1} \hat{\mu}_{i} \leq 1$. This implies $\hat{\mu}$ can have at most $\beta(G)-1$ nonzero parts, as claimed.

Once one knows $\hat{\mu}$ takes this form, and since (26) fixes the shape of $\lambda$ in its last two rows $\beta(G)-1, \beta(G)$, any LR-tableau of shape $\lambda / \mu$ and content $\left.\nu=(k(p)-1)^{\beta(G)-2}, 1\right)$ is completely determined by column-strictness and the Yamanouchi condition: it must have its unique entry equal to $\beta(G)-1$ lying in the unique of cell of $\lambda / \mu$ within the last two rows, while all of its entries in row $i$ are all equal to $i$ for $i=1,2, \ldots, \beta(G)-2$. This again forces $\lambda_{i}=\mu_{i}+k(p)$ for $i=1,2, \ldots, \beta(G)-2$, and means that $\lambda$ again matches the type of the 2-primary component on the right side of (25).

Remark 7.1. In light of what Corollary 1.4 says about $K:=K$ (line $G$ ) for nonbipartite regular graphs, one might wonder what can be deduced for bipartite regular graphs using Theorems 1.2 and 1.3. We discuss this briefly here.

Fixing a prime $p$, define $k$ to be the largest exponent such that $p^{k}$ divides $d$, and let $\operatorname{Syl}_{p}(K(G))$ have type $\mu$. Then the $p$-primary components in the bipartite case of Theorem 1.3 form the following exact sequence:

$$
0 \rightarrow \underbrace{\mathbb{Z}_{p^{k}}^{\beta(G)-1}}_{\text {type } \nu=\left(k^{\beta(G)-1}\right)} \longrightarrow \underbrace{\operatorname{Syl}_{p} K}_{\text {type } \lambda} \longrightarrow \underbrace{\operatorname{Syl}_{p}(K(\operatorname{sd} G))}_{\begin{array}{c}
\text { type } \mu \\
\text { type } \mu+\left(1^{\beta(G)}\right) \quad \begin{array}{l}
\text { if } p \neq 2 \\
\text { if } p=2
\end{array}
\end{array}} \stackrel{\pi}{\longrightarrow} \underbrace{\mathbb{Z}_{p^{k}}}_{\text {type }(k)} \rightarrow 0
$$

As a consequence, $\operatorname{Syl}_{p}(K)$ will be uniquely determined by $\operatorname{Syl}_{p}(K(G))$ whenever $p$ does not divide $d$, since then $k=0$ and (31) shows $\operatorname{Syl}_{p}(K) \cong \operatorname{Syl}_{p}(K(\operatorname{sd} G))$ in this case. However, in general, the structures of $\operatorname{ker}(\pi)$ and of $\operatorname{Syl}_{p}(K)$ seem less clear. Even using the extra information from Theorem 1.2 that $K / p^{k(p)} K \cong \mathbb{Z}_{p^{k(p}}^{\beta(G)-1} \oplus \mathbb{Z}_{\operatorname{gcd}\left(p^{k},|V|\right)}$, where $k(p)$ is the largest power such that $p^{k(p)}$ divides $2 d$, along with the LR-rule, the structures of the various terms in the sequence are not uniquely determined.

Question 7.2. When $G$ is a simple, bipartite, regular graph, what more can be said about the structure of $K:=K($ line $G)$ in relation to that of $K(G)$ ?

\section{Proof of Theorem 1.5}

Let $G=(V, E)$ be a semiregular bipartite graph with vertex bipartition $V=V_{1} \sqcup V_{2}$, such that vertices in $V_{i}$ have degree $d_{i}$. In this section we prove our analogue of Theorem 1.3 for semiregular graphs. Recall that this is motivated by Cvetković's formula (3) for the spanning tree number of line $G$ :

$$
\kappa(\text { line } G)=\frac{\left(d_{1}+d_{2}\right)^{\beta(G)}}{d_{1} d_{2}}\left(\frac{d_{1}}{d_{2}}\right)^{\left|V_{2}\right|-\left|V_{1}\right|} \kappa(G) .
$$


We recall here the statement of Theorem 1.5.

Theorem 1.5. Let $G$ be a connected bipartite $\left(d_{1}, d_{2}\right)$-semiregular graph $G$. Then there is a group homomorphism

$$
K(\text { line } G) \stackrel{g}{\rightarrow} K(G)
$$

whose kernel-cokernel exact sequence

$$
0 \rightarrow \operatorname{ker}(g) \rightarrow K(\text { line } G) \stackrel{g}{\rightarrow} K(G) \rightarrow \operatorname{coker}(g) \rightarrow 0
$$

has

- $\operatorname{coker}(g)$ all $\operatorname{lcm}\left(d_{1}, d_{2}\right)$-torsion, and

- $\operatorname{ker}(g)$ all $\frac{d_{1}+d_{2}}{\operatorname{gcd}\left(d_{1}, d_{2}\right)} \operatorname{lcm}\left(d_{1}, d_{2}\right)$-torsion.

The proof of this result is analogous to that of Theorem 1.3; for this reason, some proofs here are either abbreviated or only sketched. Note also that this theorem is less precise than Theorem 1.3, partly out of necessity: Examples 8.6 and 8.7 below show that the morphism $g: K($ line $G) \rightarrow K(G)$ appearing in the theorem is nearly surjective in some cases, and is the zero morphism in some other cases!

8.1. Defining the morphism $g$. We define $g$ similarly to the map $f$ from Definition 6.1. Let

$$
\begin{aligned}
& \lambda:=\operatorname{lcm}\left(d_{1}, d_{2}\right) \\
& \gamma:=\operatorname{gcd}\left(d_{1}, d_{2}\right) .
\end{aligned}
$$

As a notational convenience, denote typical vertices in $V_{1}$ (respectively, $V_{2}$ ) by $a$ 's (respectively, $b$ 's) with subscripts or primes.

Definition 8.1. For a semiregular bipartite graph $G$, let $g: \mathbb{R}^{E_{\text {line } G}} \rightarrow \mathbb{R}^{E_{G}}$ be defined $\mathbb{R}$-linearly by

$$
\begin{aligned}
& g\left(a b, b a^{\prime}\right)=\frac{\lambda}{d_{2}}\left((a, b)+\left(b, a^{\prime}\right)\right) \\
& g\left(b a, a b^{\prime}\right)=\frac{\lambda}{d_{1}}\left((b, a)+\left(a, b^{\prime}\right)\right) .
\end{aligned}
$$

Equivalently, the adjoint map $g^{t}$ is defined by

$$
g^{t}(a, b)=\frac{\lambda}{d_{1}} \sum_{b_{i} \in N(a)}\left(b_{i} a, a b\right)+\frac{\lambda}{d_{2}} \sum_{a_{j} \in N(b)}\left(a b, b a_{j}\right),
$$

where $N(v)$ denotes the set of vertices adjacent to $v$ in $G$.

Remark 8.2. In the special case when $G$ is not only semiregular bipartite, but actually regular, so $d_{1}=d_{2}=\lambda=\gamma$, one can easily check that the map $g$ coincides with the composite map $h \circ f$

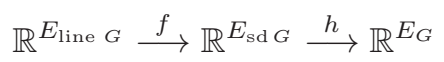

where $f$ is the map from Theorem 1.3 defined in Definition 6.1, and $h$ was defined in Example 3.3.

Proposition 8.3. If $G$ is a semiregular bipartite graph, then $g: \mathbb{Z}^{E_{\text {line }}} \rightarrow \mathbb{Z}^{E_{G}}$ is a morphism of the associated rational orthgonal decompositions, and hence induces a group homomorphism $g: K($ line $G) \rightarrow K(G)$. 
Proof. By Lemma 6.3 (ii), it is enough to show that $g$ takes global and local cycles in $Z_{\text {line } G}$ to cycles in $Z_{G}$, and that $g^{t}$ takes cycles in $Z_{G}$ to cycles in $Z_{\text {line } G}$.

First, one can check that $g$ maps all local cyles to 0 . Each global cycle is by definition of the form $z$ (line $C$ ) where $C$ is a directed cycle of $G$, and one checks that

$$
g(z(\text { line } C))=\left(\frac{\lambda}{d_{1}}+\frac{\lambda}{d_{2}}\right) z(C) .
$$

On the other hand, one checks that $g^{t}(z(C))$ can be rewritten as a sum of $\lambda$ cycles $\zeta_{i}$ in $Z_{\text {line } G}$, each $\zeta_{i}$ being twice the length of $C$, and in which every other vertex on $\zeta_{i}$ corresponds to an edge occurring in $C$.

\subsection{Analyzing its kernel and cokernel.}

Proposition 8.4. The map

$$
g^{t} g: K(\text { line } G) \rightarrow K(\text { line } G)
$$

coincides with scalar multiplication by $\frac{d_{1}+d_{2}}{\gamma} \lambda$. Consequently, $\operatorname{ker}(g)$ is $\frac{d_{1}+d_{2}}{\gamma} \lambda$-torsion.

Proof. For any edge $a b, b a^{\prime}$ in $E_{\text {line } G}$, use the definitions of $g$ and $g^{t}$ to write

$$
\begin{aligned}
g^{t} g\left(a b, b a^{\prime}\right)= & \frac{\lambda^{2}}{d_{1} d_{2}} \sum_{b_{i} \in N(a)}\left(b_{i} a, a b\right)+\frac{\lambda^{2}}{d_{2}^{2}} \sum_{a_{j} \in N(b)}\left(a b, b a_{j}\right) \\
& +\frac{\lambda^{2}}{d_{2}^{2}} \sum_{a_{j} \in N(b)}\left(a_{j} b, b a^{\prime}\right)+\frac{\lambda^{2}}{d_{1} d_{2}} \sum_{b_{k} \in N\left(a^{\prime}\right)}\left(b a^{\prime}, a^{\prime} b_{k}\right) .
\end{aligned}
$$

For the first and fourth term, one has

$$
\begin{aligned}
\sum_{b_{i} \in N(a)}\left(b_{i} a, a b\right) & =\sum_{a_{j} \in N(b)}\left(a b, b a_{j}\right) \bmod B_{\text {line } G} \\
\sum_{b_{k} \in N\left(a^{\prime}\right)}\left(b a^{\prime}, a^{\prime} b_{k}\right) & =\sum_{a_{j} \in N(b)}\left(a_{j} b, b a^{\prime}\right) \bmod B_{\text {line } G .} .
\end{aligned}
$$

Substituting these expressions into equation (33), grouping like terms, and using the identity $d_{1} d_{2}=\lambda \gamma$ gives

$$
g^{t} g\left(a b, b a^{\prime}\right)=\frac{d_{1}+d_{2}}{\gamma} \cdot \frac{\lambda}{d_{2}}\left(\sum_{a_{j} \in N(b)}\left(a b, b a_{j}\right)+\sum_{a_{j} \in N(b)}\left(a_{j} b, b a^{\prime}\right)\right) \bmod B_{\text {line } G},
$$

which then can be rewritten, using the $d_{2}$ triangular cycles

$$
\left(a b, b a_{j}\right)+\left(a_{j} b, b a^{\prime}\right)+\left(a^{\prime} b, b a\right) \in Z_{\text {line } G},
$$

as

$$
\begin{aligned}
g^{t} g\left(a b, b a^{\prime}\right) & =\frac{\left(d_{1}+d_{2}\right)}{\gamma} \cdot \frac{\lambda}{d_{2}}\left(d_{2}\left(a b, b a^{\prime}\right)\right) \bmod B_{\text {line } G}+Z_{\text {line } G} \\
& =\frac{\left(d_{1}+d_{2}\right)}{\gamma} \lambda\left(a b, b a^{\prime}\right) \bmod B_{\text {line } G}+Z_{\text {line } G} \cdot
\end{aligned}
$$

Remark 8.5. As in Proposition 6.5, one can show that the other map $g g^{t}: K(G) \rightarrow K(G)$ also coincides with the scalar multiplication by $\frac{d_{1}+d_{2}}{\gamma} \lambda$, and hence that $\operatorname{coker}(g)$ is also $\frac{d_{1}+d_{2}}{\gamma} \lambda$-torsion. However, we omit this proof, since we are about to show the stronger assertion that $\operatorname{coker}(g)$ is $\lambda$-torsion. 
Proof of Theorem 1.5. In light of Proposition 8.4, it only remains to show that $\operatorname{coker}(g)$ is $\lambda$-torsion. Given any edge $a b \in E_{G}$, one has

$$
\begin{aligned}
\lambda(a, b) & =\lambda(a, b)+\frac{\lambda}{d_{2}} \sum_{a_{j} \in N(b)}\left(b, a_{j}\right) \bmod B_{G} \\
& =\frac{\lambda}{d_{2}} \sum_{a_{j} \in N(b)}(a, b)+\left(b, a_{j}\right) \bmod B_{G} \\
& =g\left(\sum_{a_{j} \in N(b)}\left(a b, b a_{j}\right)\right) \bmod B_{G} .
\end{aligned}
$$

Consequently $\lambda(a, b)$ lies in $\operatorname{im}(g)+B_{G}$, so it is zero in $\operatorname{coker}(g):=\mathbb{Z}^{E_{G}} /\left(\operatorname{im}(g)+B_{G}+Z_{G}\right)$.

Unlike the map $f$ from Section 6 , it is hard to be much more precise about the exact nature of cokernel and kernel of $g$. The following two families of examples demonstrate two extremes of behavior for how tightly or loosely the map $g$ ties together $K($ line $G$ ) and $K(G)$ for semiregular bipartite graphs $G$.

Example 8.6. Assume $G$ is not only bipartite semiregular, but actually $d$-regular (i.e., $\left.d_{1}=d_{2}=d\right)$. Then $g: K($ line $G) \rightarrow K(G)$ is nearly surjective, in the sense that coker $(g)$ is a quotient of $\mathbb{Z}_{d}$. To see this, recall from Remark 8.2 that in this case, $g=h \circ f$ where $h, f$ were defined in Example 3.3 and Definition 6.1. Since $h: K(\operatorname{sd} G) \rightarrow K(G)$ is surjective, it induces a surjection

$$
\operatorname{coker}(f):=K(\operatorname{sd} G) / \operatorname{im}(f) \longrightarrow K(G) / \operatorname{im}(h \circ f)=: \operatorname{coker}(g) .
$$

But Theorem 1.3 says that $\operatorname{coker}(f)=\mathbb{Z}_{d}$ in this situation.

Example 8.7. For the complete bipartite graph $G=K_{n_{1}, n_{2}}$, the structures of the critical groups of $G$ and line $G$ have been determined through manipulations of their Laplacian matrices (see Lorenzini [12] and Berget [3], respectively):

$$
\begin{aligned}
K\left(K_{n_{1}, n_{2}}\right) & \cong \mathbb{Z}_{n_{1}}^{n_{2}-2} \oplus \mathbb{Z}_{n_{2}}^{n_{1}-2} \oplus \mathbb{Z}_{n_{1} n_{2}}, \\
K\left(\text { line } K_{n_{1}, n_{2}}\right) & \cong \mathbb{Z}_{n_{1}\left(n_{1}+n_{2}\right)}^{n_{1}-2} \oplus \mathbb{Z}_{n_{2}\left(n_{1}+n_{2}\right)}^{n_{2}-2} \oplus \mathbb{Z}_{n_{1}+n_{2}}^{\left(n_{1}-2\right)\left(n_{2}-2\right)+1} .
\end{aligned}
$$

In principle, the structures of these groups allow nonzero homomorphisms between them for all values of $n_{1}, n_{2}$. However, we claim that whenever $\gamma=\operatorname{gcd}\left(n_{1}, n_{2}\right)=1$, the map $K($ line $G) \stackrel{g}{\rightarrow} K(G)$ will be the zero morphism. In this case, $\operatorname{ker}(g)=K($ line $G$ ) and $\operatorname{coker}(g)=K(G)$.

To see this claim, let $\left(a b, b a^{\prime}\right)$ be a fixed edge in $E_{\text {line }} G$. Note that

$$
\begin{aligned}
\lambda & =d_{1} d_{2}=n_{1} n_{2}, \\
d_{1} & =n_{2}, d_{2}=n_{1} .
\end{aligned}
$$

Then for each $b_{j} \in V_{2}$, one has

$$
\frac{1}{d_{1}}\left(g\left(a b, b a^{\prime}\right)+g\left(a^{\prime} b_{j}, b_{j} a\right)\right)=a b+b a^{\prime}+a^{\prime} b_{j}+b_{j} a \in Z_{G} .
$$


On the other hand,

$$
\begin{aligned}
\sum_{b_{j} \in V_{2}} \frac{1}{d_{1}}\left(g\left(a b, b a^{\prime}\right)+g\left(a^{\prime} b_{j}, b_{j} a\right)\right) & =g\left(a b, b a^{\prime}\right)+\sum_{b_{j} \in V_{2}} \frac{1}{d_{1}} \frac{\lambda}{d_{2}}\left(a^{\prime} b_{j}+b_{j} a\right) \\
& =g\left(a b, b a^{\prime}\right)+\sum_{b_{j} \in V_{2}} a^{\prime} b_{j}+\sum_{b_{j} \in V_{2}} b_{j} a \\
& =g\left(a b, b a^{\prime}\right) \bmod B_{G} .
\end{aligned}
$$

Combining these two statements gives us $g\left(a b, b a^{\prime}\right)=0 \bmod Z_{G}+B_{G}$. By symmetry, one also has $g\left(b a, a b^{\prime}\right)=0 \bmod Z_{G}+B_{G}$. It follows that $g$ is the zero morphism.

Remark 8.8. Note that Theorem 1.2 provides convenient information about $\operatorname{Syl}_{p}(K)$ for $K:=K($ line $G)$ when $G$ is $\left(d_{1}, d_{2}\right)$-semiregular: if $k(p)$ denotes the largest power $p^{k(p)}$ dividing $d_{1}+d_{2}$, then

$$
K / p^{k(p)} K \cong \mathbb{Z}_{p^{k(p)}}^{\beta(G)-1} \oplus \mathbb{Z}_{\operatorname{gcd}\left(p^{k(p)},|V|\right)} .
$$

However, even in conjunction with Theorem 1.5, this does not appear to determine the structure of $K$ (line $G$ ) uniquely in terms of the structure of $K(G)$. Thus we are led to the following generalization of Question 7.2:

Question 8.9. When $G$ is a simple, semiregular bipartite graph, what more can be said about the structure of $K:=K($ line $G$ ) in relation to that of $K(G)$ ?

\section{ExAmples}

9.1. The complete graph $K_{n}$. Proposition 3.3 can be rephrased as asserting that

$$
K\left(K_{n}\right) \cong \mathbb{Z}_{n}^{n-2} \oplus \mathbb{Z}_{1}^{\beta-n}
$$

where $\beta:=\beta\left(K_{n}\right)=\left(\begin{array}{c}n-1 \\ 2\end{array}\right)$. Since $K_{n}$ is nonbipartite for $n \geq 3$, and contains an even length cycle for $n \geq 4$, Corollary 1.4 immediately implies the following:

Corollary 9.1. For $n \geq 4$, the line graph line $K_{n}$ of the critical group of the complete graph $K_{n}$ has the form

$$
\begin{aligned}
& K\left(\text { line } K_{4}\right)=\mathbb{Z}_{24} \oplus \mathbb{Z}_{8} \oplus \mathbb{Z}_{2} \\
& K\left(\text { line } K_{n}\right)=\mathbb{Z}_{2(n-1) n}^{n-2} \oplus \mathbb{Z}_{2(n-1)}^{\beta-n} \oplus \begin{cases}\mathbb{Z}_{2}^{2} & \text { for even } n>5, \\
\mathbb{Z}_{4} & \text { for odd } n \geq 5 .\end{cases}
\end{aligned}
$$

9.2. The complete bipartite graph $K_{n_{1}, n_{2}}$. As mentioned in Example 8.7, the critical groups of the complete bipartite graph $K_{n_{1}, n_{2}}$ and its line graph line $K_{n_{1}, n_{2}}$ have the following forms:

$$
\begin{aligned}
K\left(K_{n_{1}, n_{2}}\right) & \cong \mathbb{Z}_{n_{1}}^{n_{2}-2} \oplus \mathbb{Z}_{n_{2}}^{n_{1}-2} \oplus \mathbb{Z}_{n_{1} n_{2}} \\
K\left(\text { line } K_{n_{1}, n_{2}}\right) & \cong \mathbb{Z}_{n_{1}\left(n_{1}+n_{2}\right)}^{n_{1}-2} \oplus \mathbb{Z}_{n_{2}\left(n_{1}+n_{2}\right)}^{n_{2}-2} \oplus \mathbb{Z}_{n_{1}+n_{2}}^{\left(n_{1}-2\right)\left(n_{2}-2\right)+1}
\end{aligned}
$$

(see Lorenzini [12] and Berget [3], respectively).

In addition, Example 8.7 showed that the map $g$ in the exact sequence in Theorem 1.5 is sometimes the zero morphism and hence is not always useful for determining the structure of 
$K$ (line $K_{n_{1}, n_{2}}$ ). Even in the special case when $n_{1}=n_{2}=n$ (so $K$ (line $\left.K_{n, n}\right)$ is $n$-regular), the exact sequence

$$
0 \rightarrow \underbrace{\mathbb{Z}_{n}^{\beta-2} \oplus \mathbb{Z}_{n}}_{\mathbb{Z}_{n}^{n(n-2)}} \longrightarrow \underbrace{K\left(\text { line } K_{n, n}\right)}_{\mathbb{Z}_{2 n^{2}}^{2(n-2)} \oplus \mathbb{Z}_{2 n}^{(n-2)^{2}+1}} \stackrel{f}{\longrightarrow} \underbrace{K\left(\operatorname{sd} K_{n, n}\right)}_{\mathbb{Z}_{2 n^{2}}^{1} \oplus \mathbb{Z}_{2 n}^{2(n-2)} \oplus \mathbb{Z}_{2}^{(n-2)^{2}}} \longrightarrow \mathbb{Z}_{n} \rightarrow 0
$$

from Theorem 1.3 does not determine a priori Berget's formula for $K\left(\right.$ line $\left.K_{n, n}\right)$.

However, we note that at least Theorem 1.2 does predict that the expression

$$
K\left(\text { line } K_{n_{1}, n_{2}}\right)=\bigoplus_{i=1}^{\beta} \mathbb{Z}_{e_{i}} \text { where } \beta:=\beta\left(K_{n_{1}, n_{2}}\right)=\left(n_{1}-1\right)\left(n_{2}-1\right)
$$

should have $|V|=n_{1}+n_{2}$ dividing every one of the factors $e_{i}$. This follows from equation (34), since $K_{n_{1}, n_{2}}$ is bipartite $\left(n_{1}, n_{2}\right)$-semiregular. Hence for each prime $p$, one has

$$
K / p^{k(p)} K \cong \mathbb{Z}_{p^{k(p)}}^{\beta-1} \oplus \mathbb{Z}_{\operatorname{gcd}\left(p^{k(p)},|V|\right)}=\mathbb{Z}_{p^{k(p)}}^{\beta},
$$

where $k(p)$ is the largest power such that $p^{k(p)}$ divides $n_{1}+n_{2}$. Hence $K /\left(n_{1}+n_{2}\right) K \cong$ $\mathbb{Z}_{n_{1}+n_{2}}^{\beta}$.

9.3. The $d$-dimensional cube. Denote by $G_{\mathrm{d} \text {-cube }}$ the graph of vertices and edges in the $d$-dimensional cube, that is, $G_{\mathrm{d} \text {-cube }}=(V, E)$ in which $V$ is the set of all binary strings of length $d$, and $E$ has an edge between any two such strings that differ in exactly one binary digit. This is a $d$-regular bipartite graph, having

$$
\beta:=\beta\left(G_{\mathrm{d} \text {-cube }}\right)=(d-2) 2^{d-1}+1 .
$$

One knows its spanning tree number (see, e.g., [16, Example 5.6.10]):

$$
\kappa\left(G_{\text {d-cube }}\right)=\frac{1}{2^{d}} \prod_{k=1}^{d}(2 k)^{\left(\begin{array}{l}
d \\
k
\end{array}\right)}=2^{2^{d}-d-1} \prod_{k=2}^{d} k^{\left(\begin{array}{l}
d \\
k
\end{array}\right)} .
$$

Correspondingly, work of $\mathrm{H}$. Bai [2] computes its critical group structure away from the prime 2: For odd primes $p$, one has

$$
\operatorname{Syl}_{p}\left(K\left(G_{\text {d-cube }}\right)\right)=\operatorname{Syl}_{p}\left(\bigoplus_{k=2}^{d} \mathbb{Z}_{k}^{\left(\begin{array}{l}
d \\
k
\end{array}\right)}\right) .
$$

Unfortunately, $\operatorname{Syl}_{2}\left(K\left(G_{\mathrm{d} \text {-cube }}\right)\right)$ is a 2 -group that is still not known for all $d$.

Consequently, Proposition 3.2 shows that $K\left(\operatorname{sd}\left(G_{\text {d-cube }}\right)\right)$ has the same $p$-primary structure as $K\left(G_{\mathrm{d} \text {-cube }}\right)$ for odd primes $p$, and Theorem 1.3 gives the following exact sequence for every odd prime $p$ :

$$
0 \rightarrow \operatorname{Syl}_{p}\left(\mathbb{Z}_{d}^{\beta-1}\right) \rightarrow \operatorname{Syl}_{p}\left(K\left(\text { line } G_{\text {d-cube }}\right)\right) \rightarrow \operatorname{Syl}_{p}\left(\bigoplus_{k=2}^{d} \mathbb{Z}_{k}^{\left(\begin{array}{l}
d \\
k
\end{array}\right)}\right) \rightarrow \mathbb{Z}_{d} \rightarrow 0 .
$$

This is particularly effective when $d$ itself is prime since then $\operatorname{Syl}_{d}\left(\bigoplus_{k=2}^{d} \mathbb{Z}_{k}^{\left(\begin{array}{l}d \\ k\end{array}\right)}\right)=\mathbb{Z}_{d}$ and the exact sequence (35) implies that for odd primes $p$,

$$
\operatorname{Syl}_{p}\left(K\left(\text { line } G_{\text {d-cube }}\right)\right)= \begin{cases}\mathbb{Z}_{d}^{\beta-1} & \text { if } p=d, \\
\operatorname{Syl}_{p}\left(\bigoplus_{k=2}^{d-1} \mathbb{Z}_{k}^{\left(\begin{array}{l}
d \\
k
\end{array}\right)}\right) & \text { if } p \neq d .\end{cases}
$$


Meanwhile $\operatorname{Syl}_{2}\left(K\left(\right.\right.$ line $\left.\left.G_{\text {d-cube }}\right)\right)=\operatorname{Syl}_{2}\left(K\left(\operatorname{sd}\left(G_{\mathrm{d} \text {-cube }}\right)\right)\right)$ is unknown, but by Proposition 3.2 , is completely determined by the unknown 2 -group $\operatorname{Syl}_{2}\left(K\left(G_{\mathrm{d} \text {-cube }}\right)\right)$.

9.4. The Platonic solids. One source of regular graphs are the 1-skeleta (= graph of vertices and edges) of the Platonic solids. There are certain features that apply to any graph $G_{P}$ which is the 1-skeleton of a 3-dimensional polyhedron $P$, and hence to any Platonic solid:

- Because the cycles surrounding the (polygonal) faces of $P$ generate the cycle lattice $Z$, the graph $G_{P}$ is bipartite if and only if each face of $P$ is an even $n$-gon.

- Furthermore, the cycles that bound all but one face of $P$ form a basis for $Z$, so that $\beta\left(G_{P}\right)$ is always one less than the number of faces.

- Such graphs $G_{P}$ are always 2-edge-connected, so that Theorem 1.1 always applies.

- Dual polyhedra $P, P^{*}$ have $G_{P}, G_{P^{*}}$ dual as planar graphs. This identifies the lattice of bonds for one with the lattice of cycles for the other, and implies that their critical groups $K\left(G_{P}\right), K\left(G_{P *}\right)$ are isomorphic; see also [6].

9.4.1. The tetrahedron. The tetrahedron has 1-skeleton $G_{\text {tetra }}=K_{4}$, and hence implicitly was discussed already in Section 9.1 on $K_{n}$, as the special case $n=4$.

9.4.2. The cube and octahedron. The cube and the octahedron are dual polyhedra. Either by direct computer calculation, or by noting $G_{\text {octa }} \cong$ line $K_{4}$ and applying Corollary 9.1 with $n=4$, one finds that

$$
\begin{aligned}
K\left(G_{\text {cube }}\right)=K\left(G_{\text {octa }}\right) & =\mathbb{Z}_{2} \oplus \mathbb{Z}_{8} \oplus \mathbb{Z}_{24} \\
& =\mathbb{Z}_{2} \oplus \mathbb{Z}_{8}^{2} \oplus \mathbb{Z}_{3} .
\end{aligned}
$$

Since $G_{\text {octa }}$ is 4-regular and nonbipartite with $\beta\left(G_{\text {octa }}\right)=7$, Corollary 1.4 then implies

$$
K\left(\text { line } G_{\text {octa }}\right)=\mathbb{Z}_{2}^{2} \oplus \mathbb{Z}_{8}^{2} \oplus \mathbb{Z}_{16} \oplus \mathbb{Z}_{64} \oplus \mathbb{Z}_{192} \text {. }
$$

For $G_{\text {cube }}$, which has $\beta\left(G_{\text {cube }}\right)=5$, the results of Section 9.3 apply, and are particularly effective because $d=3$ is prime. They show that $\operatorname{Syl}_{p}\left(K\left(\right.\right.$ line $\left.\left.G_{\mathrm{d} \text {-cube }}\right)\right)$ vanishes except for $p=2,3$, with

$$
\begin{aligned}
& \operatorname{Syl}_{3}\left(K\left(\text { line } G_{\text {d-cube }}\right)\right)=\mathbb{Z}_{3}^{4} \\
& \operatorname{Syl}_{2}\left(K\left(\text { line } G_{\text {d-cube }}\right)\right)=\operatorname{Syl}_{2}\left(K\left(\operatorname{sd}\left(G_{\text {d-cube }}\right)\right)\right)=\mathbb{Z}_{2}^{2} \oplus \mathbb{Z}_{4} \oplus \mathbb{Z}_{16}^{2} .
\end{aligned}
$$

Hence

$$
\begin{aligned}
K\left(\text { line } G_{\text {cube }}\right) & =\mathbb{Z}_{2}^{2} \oplus \mathbb{Z}_{4} \oplus \mathbb{Z}_{16}^{2} \oplus \mathbb{Z}_{3}^{4} \\
& =\mathbb{Z}_{2} \oplus \mathbb{Z}_{6} \oplus \mathbb{Z}_{12} \oplus \mathbb{Z}_{48}^{2} .
\end{aligned}
$$

9.4.3. The dodecahedron and icosahedron. The dodecahedron and icosahedron are dual polyhedra, both of whose graphs are nonbipartite. Computer calculation shows that

$$
\begin{aligned}
K\left(G_{\text {dodeca }}\right)=K\left(G_{\text {icosa }}\right) & =\mathbb{Z}_{2} \oplus \mathbb{Z}_{12} \oplus \mathbb{Z}_{60}^{3} \\
& =\mathbb{Z}_{2} \oplus \mathbb{Z}_{4}^{4} \oplus \mathbb{Z}_{3}^{4} \oplus \mathbb{Z}_{5}^{3} .
\end{aligned}
$$

Since $G_{\text {dodeca }}$ is 5 -regular with $\beta\left(G_{\text {dodeca }}\right)=11$, one concludes from Corollary 1.4 that

$$
\begin{aligned}
K\left(\text { line } G_{\text {dodeca }}\right) & =\mathbb{Z}_{2}^{2} \oplus \mathbb{Z}_{6}^{4} \oplus \mathbb{Z}_{12} \oplus \mathbb{Z}_{72} \oplus \mathbb{Z}_{360}^{3} \\
& =\mathbb{Z}_{2}^{6} \oplus \mathbb{Z}_{4} \oplus \mathbb{Z}_{8}^{4} \oplus \mathbb{Z}_{3}^{5} \oplus \mathbb{Z}_{9}^{4} \oplus \mathbb{Z}_{5}^{3} .
\end{aligned}
$$

Since $G_{\text {icosa }}$ is 3 -regular with $\beta\left(G_{\text {icosa }}\right)=19$, one concludes from Corollary 1.4 that

$$
\begin{aligned}
K\left(\text { line } G_{\text {icosa }}\right) & =\mathbb{Z}_{2}^{2} \oplus \mathbb{Z}_{10}^{12} \oplus \mathbb{Z}_{20} \oplus \mathbb{Z}_{120} \oplus \mathbb{Z}_{600}^{3} \\
& =\mathbb{Z}_{2}^{14} \oplus \mathbb{Z}_{4} \oplus \mathbb{Z}_{8}^{4} \oplus \mathbb{Z}_{3}^{4} \oplus \mathbb{Z}_{5}^{14} \oplus \mathbb{Z}_{25}^{3} .
\end{aligned}
$$




\section{REFERENCES}

[1] Bacher, de la Harpe, and Nagnibeda, The lattice of integral cuts and lattice of integral flows of a finite graph. Bull. Soc. Math. France 125 (1997), 167-198.

[2] H. Bai, On the critical group of the $n$-cube, Linear Algebra and its Applications 369 (2003), 251-261.

[3] A. Berget, Critical groups of some regular line graphs, (2003). Available online at http://www.umn.edu/ berget/research/

[4] N. Biggs, Algebraic potential theory on graphs, Bull. London Math. Soc. 29 (1997), 641-682.

[5] N. Biggs, Algebraic Graph Theory. Cambridge University Press, 1993.

[6] R. Cori and D. Rossin, On the sandpile group of dual graphs. European J. Combin. 21 (2000), no. 4, 447-459.

[7] D. Cvetković, M. Doob, and H. Sachs, Spectra of graphs. Theory and application. Pure and Applied Mathematics 87. Academic Press, Inc. [Harcourt Brace Jovanovich, Publishers], New York-London, 1980.

[8] C. Godsil and G. Royle, Algebraic Graph Theory, Springer-Verlag, 2001.

[9] N. Hartsfield and G. Ringel, Pearls in Graph Theory: A Comprehensive Introduction. Academic Press, 1994.

[10] B. Jacobson, A. Neidermeier and V. Reiner, Critical groups for complete multipartite graphs and Cartesian products of complete graphs. J. Graph Theory 44 (2003), 231-250

[11] A.K. Kelmans, Properties of the characteristic polynomial of a graph, Kibernetiky - na službu kommunizmu, 4, Energija, Moskva - Leningrad, (1967), 27-41 (in Russian).

[12] D. Lorenzini, A finite group attached to the laplacian of a graph. Discrete Mathematics 91 (1991), $277-282$.

[13] I.G. Macdonald, Symmetric functions and Hall polynomials, 2nd edition. Oxford Mathematical Monographs. Oxford Science Publications. The Clarendon Press, Oxford University Press, New York, 1995.

[14] B. Mohar, The Laplacian Spectrum of Graphs. Graph Theory, Combinatorics, and Applications 2 Ed. by Y. Alavi, G. Chartrand, O. R. Oellermann, A. J. Schwenk. Wiley, 1991, 871-898.

[15] M. Rubey, Counting Spanning Trees. Diplomarbeit, Universität Wien, 2000.

[16] R.P. Stanley, Enumerative Combinatorics, Vol. 2. Cambridge Studies in Advanced Mathematics 62. Cambridge University Press, Cambridge, 1999.

[17] R.P. Stanley, A zonotope associated with graphical degree sequences. Applied geometry and discrete mathematics, 555-570, DIMACS Ser. Discrete Math. Theoret. Comput. Sci. 4, Amer. Math. Soc., Providence, RI, 1991.

[18] D. Treumann, Functoriality of critical groups, Bachelor's thesis, Univ. of Minnesota, 2002, Available online at http://www.math.umn.edu/ reiner/REU/REU.html.

[19] E.B. Vahovskii, On the characteristic numbers of incidence matrices for non-singular graphs, Sibirsk. Mat. Zh. 6 (1965) 44-49 (in Russian).

[20] D. West, Introduction to Graph Theory. Prentice Hall, 2001.

E-mail address: berget@math.umn.edu

E-mail address: amanion1@nd.edu

E-mail address: maxwell@math.umn.edu

E-mail address: potechin@princeton.edu

E-mail address: reiner@math.umn.edu

School of Mathematics, 206 Church St. S.E., University of Minnesota, Minneapolis, Mn 55455 


\title{
THE CRITICAL GROUP OF A LINE GRAPH
}

\author{
ANDREW BERGET, ANDREW MANION, MOLLY MAXWELL, AARON POTECHIN, \\ AND VICTOR REINER
}

\begin{abstract}
The critical group of a graph is a finite abelian group whose order is the number of spanning forests of the graph. This paper provides three basic structural results on the critical group of a line graph.

- The first deals with connected graphs containing no cut-edge. Here the number of independent cycles in the graph, which is known to bound the number of generators for the critical group of the graph, is shown also to bound the number of generators for the critical group of its line graph.

- The second gives, for each prime $p$, a constraint on the $p$-primary structure of the critical group, based on the largest power of $p$ dividing all sums of degrees of two adjacent vertices.

- The third deals with connected graphs whose line graph is regular. Here known results relating the number of spanning trees of the graph and of its line graph are sharpened to exact sequences which relate their critical groups.

The first two results interact extremely well with the third. For example, they imply that in a regular nonbipartite graph, the critical group of the graph and that of its line graph determine each other uniquely in a simple fashion.
\end{abstract}

\section{Contents}

1. Introduction and main results

1.1. The hypothesis of no cut-edge

1.2. The hypothesis that degree sums of adjacent vertices are divisible by $p$

1.3. The regularity hypothesis

2. Some theory of lattices

2.1. Rational orthogonal decompositions

2.2. Functoriality

2.3. Pontrjagin duality

3. The critical group of a graph

3.1. Cycles, bonds, Laplacians, and spanning trees 9

3.2. A presentation for $K($ line $G) \quad 10$

3.3. Lorenzini's result on edge subdivisions 10

3.4. A non-standard treatment of the complete graph 11

4. Proof of Theorem 1.1 12

5. Proof of Theorem $1.2 \quad 15$

6. Proof of Theorem $1.3 \quad 19$

$\begin{array}{ll}\text { 6.1. } & \text { Defining the morphism } f \\ & 19\end{array}$

6.2. The kernel and cokernel of $f$ are $d$-torsion 21

Key words and phrases. Critical group, line graph, regular graph.

Work of all authors supported by NSF grants DMS-0245379 and DMS-0601010, and completed partly during REU programs at the University of Minnesota during the summers of 2003, 2004 and 2008. 
6.3. Analyzing the cokernel 22

6.4. Analyzing the kernel 23

$\begin{array}{lr}\text { 7. Proof of Corollary 1.4 } & 26\end{array}$

$\begin{array}{ll}\text { 8. Proof of Theorem 1.5 } & 28\end{array}$

8.1. Defining the morphism $g \quad 29$

8.2. Analyzing its kernel and cokernel 30

9. Examples 32

9.1. The complete graph $K_{n} \quad 32$

9.2. The complete bipartite graph $K_{n_{1}, n_{2}}$

9.3. The $d$-dimensional cube 33

9.4. The Platonic solids $\quad 34$

Acknowledgments $\quad 35$

$\begin{array}{ll}\text { References } & 35\end{array}$

\section{INTRODUCTION AND MAIN RESULTS}

The critical group $K(G)$ of a graph $G$ is a finite abelian group whose order is the number $\kappa(G)$ of spanning forests ${ }^{1}$ of the graph. One can define $K(G)$ in several ways, closely related to the cycle and bond spaces of the graph, the graph Laplacian, as well as a certain chipfiring game that is played on the vertices of the graph and is called the abelian sandpile model in the physics literature. The interested reader can find some of the standard results on $K(G)$ in [1, 4] and [8, Chapter 13]. Some of this material is reviewed in Sections 2 and 3 below, along with unpublished results from the bachelor's thesis of D. Treumann [19] on functoriality for critical groups.

The critical group $K(G)$ and its relation to the structure of the graph $G$ remain, in general, mysterious. The goal of this paper is to compare the structure of the critical group of a connected simple graph (that is, a connected graph having no multiple edges and no loops) with that of the critical group of its line graph. Recall that for a graph $G=(V, E)$, its line graph line $G=\left(V_{\text {line } G}, E_{\text {line } G}\right)$ has vertex set $V_{\text {line } G}:=E$, the edge set of $G$, and an edge in $E_{\text {line } G}$ corresponding to each pair of edges in $E$ that are incident at a vertex. Our
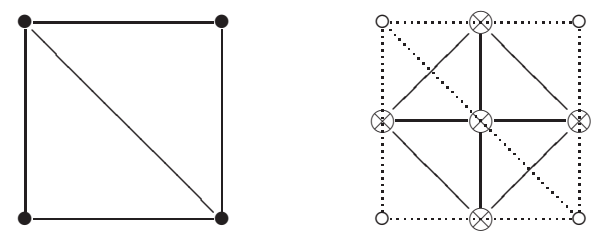

Figure 1. A graph $G$ and its line graph line $G$ with $G$ underlayed.

main results say that, under three different kinds of hypotheses, the structure of $K$ (line $G$ ) is not much more complicated than that of $K(G)$, as we now explain.

\footnotetext{
${ }^{1}$ Throughout this paper all spanning forests are assumed to be maximal in the sense that adding an edge of $G$ to a spanning forest creates a cycle. This removes the possibility of a connected graph containing a disconnected spanning forest.
} 
1.1. The hypothesis of no cut-edge. It is well-known, and follows from one of the definitions of $K(G)$ in Section 3, that for a connected graph $G$, the number $\beta(G):=|V|-|E|+1$ of independent cycles in $G$ gives an upper bound on the number of generators required for $K(G)$; that is,

$$
K(G)=\bigoplus_{i=1}^{\beta(G)} \mathbb{Z}_{d_{i}}
$$

where $\mathbb{Z}_{d}$ denotes the cyclic group $\mathbb{Z} / d \mathbb{Z}$ (not the $d$-adic integers), and the $d_{i}$ are positive integers (some of which may be 1 ). Our first main result asserts that the same bound on the number of generators holds for $K$ (line $G$ ) when one assumes that $G$ is 2-edge-connected, that is, $G$ is connected and contains no cut-edge.

Theorem 1.1. When the simple graph $G$ is 2-edge-connected, the critical group $K$ (line $G$ ) can be generated by $\beta(G)$ elements.

Note that one needs some hypothesis on the graph $G$ for this conclusion to hold. For example, a star graph $K_{1, n}$ (= one vertex of degree $n$ connected to $n$ vertices of degree one) has $\beta\left(K_{1, n}\right)=0$. However, its line graph is the complete graph $K_{n}$ and thus, according to Proposition 3.2 below, has critical group $K\left(\right.$ line $\left.K_{1, n}\right)=\mathbb{Z}_{n}^{n-2}$, requiring $n-2$ generators. Theorem 1.1 is proven in Section 4, using a useful presentation of $K$ (line $G$ ) given in Section 3.2.

1.2. The hypothesis that degree sums of adjacent vertices are divisible by $p$. As $K($ line $G$ ) is a finite abelian group, its structure is completely determined once one knows, for each prime $p$, the structure of its $p$-primary component or $p$-Sylow subgroup $\operatorname{Syl}_{p}(K($ line $G)$ ). Section 5 below proves the following stringent constraint on this $p$-primary structure, based on the largest power $k(p)$ such that $p^{k(p)}$ divides all of the $\operatorname{sums} \operatorname{deg}_{G}(v)+\operatorname{deg}_{G}(w)$ as one runs through all edges $e=\{v, w\}$ in the edge set $E$ of $G$. Here $\operatorname{deg}_{G}(v)$ is the number of edges of $G$ with $v$ as an endpoint; it is the degree of the vertex $v$.

Theorem 1.2. Let $G=(V, E)$ be a connected simple graph that contains at least one cycle of even ${ }^{2}$ length. Use the abbreviated notation $K:=K($ line $G)$, and let $p$ be a prime for which the quantity $k(p) \geq 1$.

Then for $G$ bipartite, one has

$$
K / p^{k(p)} K \cong \mathbb{Z}_{p^{k(p)}}^{\beta(G)-1} \oplus \mathbb{Z}_{\operatorname{gcd}\left(p^{k},|V|\right)},
$$

while for $G$ nonbipartite, one has

$$
K / p^{k(p)} K \cong \mathbb{Z}_{p^{k(p)}}^{\beta(G)-2} \oplus \begin{cases}0 & \text { if } p \text { is odd, } \\ \mathbb{Z}_{2}^{2} & \text { if } p=2 \text { and }|V| \text { is even, } \\ \mathbb{Z}_{4} & \text { if } p=2 \text { and }|V| \text { is odd. }\end{cases}
$$

1.3. The regularity hypothesis. Our third class of main results deals with the situation where line $G$ is regular, that is, all its vertices have the same degree. Say that a graph is $d$-regular if all of its vertices have degree $d$. It is an easy exercise to check that, for connected graphs $G$, one has line $G$ regular only in these two situations:

- $G$ itself is $d$-regular. In this case, line $G$ will be $(2 d-2)$-regular.

\footnotetext{
${ }^{2}$ This even length cycle need not be minimal. For example, a connected graph with two cycles $C_{1}, C_{2}$ of odd length will also contain a cycle of even length that traverses $C_{1}$, follows a path from $C_{1}$ to $C_{2}$, then traverses $C_{2}$ and follows the same path back to $C_{1}$.
} 
- $G$ is bipartite and $\left(d_{1}, d_{2}\right)$-semiregular, meaning that its vertex bipartition $V=$ $V_{1} \sqcup V_{2}$ has all vertices in $V_{i}$ of degree $d_{i}$ for $i=1,2$. In this case, line $G$ will be $\left(d_{1}+d_{2}-2\right)$-regular.

Two classical theorems of graph spectra explain how the the numbers of spanning trees $\kappa(G)$ and $\kappa$ (line $G$ ) determine each other in this situation. The first is due originally to Vahovskii [20] and later Kelmans [11], then rediscovered by Sachs [7, §2.4], while the second is due originally to Cvetković $[16, \S 5.2]$ (see also [15, Theorem 3.9]).

Theorem. Let $G$ be a connected graph with line $G$ regular.

(Sachs) If $G$ is d-regular, then

$$
\kappa(\text { line } G)=d^{\beta(G)-2} 2^{\beta(G)} \kappa(G) .
$$

(Cvetkovic) If $G$ is bipartite and $\left(d_{1}, d_{2}\right)$-semiregular, then

$$
\kappa(\text { line } G)=\frac{\left(d_{1}+d_{2}\right)^{\beta(G)}}{d_{1} d_{2}}\left(\frac{d_{1}}{d_{2}}\right)^{\left|V_{2}\right|-\left|V_{1}\right|} \kappa(G) .
$$

These results suggest a close relationship between the critical groups $K(G)$ and $K($ line $G$ ) in both of these situations.

1.3.1. Regular graphs. We focus first on such a relation underlying Sachs' equation (2), as here one can be quite precise.

The occurrence of the factor $2^{\beta(G)} \kappa(G)$ within (2) suggests consideration of the edge subdivision graph $\operatorname{sd} G$, obtained from $G$ placing a new vertex at the midpoint of every edge of $G$. It is well-known that

$$
\kappa(\operatorname{sd} G)=2^{\beta(G)} \kappa(G),
$$

due to an obvious $2^{\beta(G)}$-to-1 surjective map from the spanning trees of $\operatorname{sd} G$ to those of $G^{3}$. Underlying this relation, Lorenzini [13] observed that the critical groups $K(\operatorname{sd} G)$ and $K(G)$ also determine each other in a trivial way: $K(G)$ has the form given in (1) if and only if for the same positive integers $d_{1}, d_{2}, \ldots, d_{\beta(G)}$ one has the following form for $K(\operatorname{sd} G)$ :

$$
K(\operatorname{sd} G)=\bigoplus_{i=1}^{\beta(G)} \mathbb{Z}_{2 d_{i}} .
$$

See Proposition 3.2 below. In light of (4), one might expect that equation (2) generalizes to a short exact sequence of the form

$$
0 \rightarrow \mathbb{Z}_{d}^{\beta(G)-2} \rightarrow K(\text { line } G) \rightarrow K(\operatorname{sd} G) \rightarrow 0
$$

where $\mathbb{Z}_{d}$ denotes a cyclic group of order $d$. This is never far from the truth. After reviewing and developing some theory of critical groups and their functoriality in Sections 2 and 3 below, we use functoriality to prove the following result in Section 6 .

Theorem 1.3. For any connected d-regular simple graph $G$ with $d \geq 3$, there is a natural group homomorphism $f: K($ line $G) \rightarrow K(\operatorname{sd} G)$ whose kernel-cokernel exact sequence takes the form

$$
0 \rightarrow \mathbb{Z}_{d}^{\beta(G)-2} \oplus C \rightarrow K(\text { line } G) \stackrel{f}{\rightarrow} K(\mathrm{sd} G) \rightarrow C \rightarrow 0
$$

\footnotetext{
${ }^{3}$ More explicitly, there are exactly $\beta(G)$ edges that do not lay on a given spanning tree of $G$. Upon subdividing, there are $2^{\beta(G)}$ ways to extend the resulting tree to a spanning tree of sd $G$.
} 
in which the cokernel $C$ is the following cyclic d-torsion group:

$$
C= \begin{cases}0 & \text { if } G \text { is non-bipartite and } d \text { is odd, } \\ \mathbb{Z}_{2} & \text { if } G \text { is non-bipartite and } d \text { is even } \\ \mathbb{Z}_{d} & \text { if } G \text { is bipartite. }\end{cases}
$$

It turns out that Theorems 1.1 and 1.2 interact very well with Theorem 1.3. When $G$ is a $d$-regular simple 2-edge-connected graph, Theorem 1.1 implies that $K$ (line $G$ ) needs at most $\beta(G)$ generators, while Proposition 3.2 implies that $K(\operatorname{sd} G)$ requires at least $\beta(G)$ generators, forcing $K$ (line $G$ ) to require either $\beta(G)-1$ or $\beta(G)$ generators. This shows that the exact sequence in Theorem 1.3 is about as far as possible from being split, and gives it extra power in determining the structure of $K$ (line $G$ ) given that of $K(G)$ (and hence also $K(\operatorname{sd} G))$.

Even more precisely, it will be shown in Section 7 that when $G$ is both $d$-regular and nonbipartite, Theorems 1.2 and 1.3 combined show that $K(G)$ and $K($ line $G$ ) determine each other uniquely in the following fashion.

Corollary 1.4. For $G$ a simple, connected, d-regular graph with $d \geq 3$ which is nonbipartite, after uniquely expressing

$$
K(G) \cong \bigoplus_{i=1}^{\beta(G)} \mathbb{Z}_{d_{i}}
$$

with $d_{i}$ dividing $d_{i+1}$, one has

$$
K(\text { line } G) \cong\left(\begin{array}{ll}
\beta(G)-2 \\
\bigoplus_{i=1} \mathbb{Z}_{2 d d_{i}}
\end{array}\right) \oplus \begin{cases}\mathbb{Z}_{2 d_{\beta(G)-1}} \oplus \mathbb{Z}_{2 d_{\beta(G)}} & \text { for }|V| \text { even }, \\
\mathbb{Z}_{4 d_{\beta(G)-1}} \oplus \mathbb{Z}_{d_{\beta(G)}} & \text { for }|V| \text { odd. }\end{cases}
$$

1.3.2. Semiregular bipartite graphs. Section 8 uses functoriality to prove the following result analogous to Theorem 1.3 and suggested by Cvetković's equation (3).

Theorem 1.5. Let $G$ be a connected bipartite $\left(d_{1}, d_{2}\right)$-semiregular graph $G$. Then there is a group homomorphism

$$
K(\text { line } G) \stackrel{g}{\rightarrow} K(G)
$$

whose kernel-cokernel exact sequence

$$
0 \rightarrow \operatorname{ker}(g) \rightarrow K(\text { line } G) \stackrel{g}{\rightarrow} K(G) \rightarrow \operatorname{coker}(g) \rightarrow 0
$$

has coker $(g)$ all $\operatorname{lcm}\left(d_{1}, d_{2}\right)$-torsion, and has $\operatorname{ker}(g)$ all $\frac{d_{1}+d_{2}}{\operatorname{gcd}\left(d_{1}, d_{2}\right)} \operatorname{lcm}\left(d_{1}, d_{2}\right)$-torsion.

Note that this result describes the kernels and cokernels less completely than Theorem 1.3. Section 8 discusses examples illustrating why this is necessarily the case.

Section 9 illustrates some of the preceding results by showing how they apply to the examples of complete graphs and complete bipartite graphs, as well as the 1-skeleta of $d$-dimensional cubes and the Platonic solids.

1.3.3. Directed line graphs. The reader should compare our results with recent results of Levine [12] on the critical group of a directed line graph. If $G$ is a directed graph, then the directed line graph $\mathcal{L} G$ is defined so that a pair of directed edges $e$ and $f$ of $G$ are adjacent (and oriented from $e$ to $f$ ) if the head of $e$ is equal to the tail $f$.

The critical group of a directed graph is defined as the torsion subgroup of the cokernel of the Laplacian matrix of $G$. Levine proves [12, Theorem 1.2] that if $G$ is a strongly connceted Eulerian directed graph, then there is a surjective group homomorphism from the critical 
group of $\mathcal{L} G$ to the critical group of $G$. Moreover, when the $G$ is balanced and $d$-regular, the kernel of this homomorphism is the $d$-tosion subgroup of the critical group of $\mathcal{L} G$. As can be seen from Theorem 1.3, we do not obtain such easily stated results in the undirected case.

\section{Some THEORY OF LATtices}

This section recalls some of the theory of rational lattices in Euclidean spaces and their determinant groups, along with functoriality and Pontrjagin duality for these groups, borrowing heavily from Bacher, de la Harpe, and Nagnibeda [1] and Treumann [19]. In the next section, these constructions will be specialized to critical groups of graphs.

2.1. Rational orthogonal decompositions. Consider $\mathbb{R}^{m}$ with its usual inner product $\langle\cdot, \cdot\rangle$ in which the standard basis vectors $e_{1}, \ldots, e_{m}$ are orthonormal. The $\mathbb{Z}$-span of this basis is the integer lattice $\mathbb{Z}^{m}$.

Definition 2.1. A rational orthogonal decomposition is an orthogonal $\mathbb{R}$-vector space decomposition of $\mathbb{R}^{m}=B^{\mathbb{R}} \oplus Z^{\mathbb{R}}$ in which $B^{\mathbb{R}}, Z^{\mathbb{R}}$ are $\mathbb{R}$-subspaces which are rational, that is, spanned by elements of $\mathbb{Z}^{m}$.

Example 2.2. The main example of interest for us will be the following, discussed further in Section 3. If $G=(V, E)$ is a graph with $|E|=m$, then the space $Z^{\mathbb{R}}$ of 1-cycles together with its orthogonal complement, the space $B^{\mathbb{R}}$ of bonds or 1-coboundaries, give a rational orthogonal decomposition $\mathbb{R}^{E} \cong \mathbb{R}^{m}=B^{\mathbb{R}} \oplus Z^{\mathbb{R}}$. Here one must fix an (arbitrary) orientation of the edges in $E$ in order to make the identification $\mathbb{R}^{E} \cong \mathbb{R}^{m}$. In the remaining sections, the basis element of $\mathbb{R}^{E}$ corresponding to an edge $\{u, v\}$ of $G$ oriented from $u$ to $v$ will sometimes be denoted $e$ and sometimes $(u, v)$, with the convention that $(v, u)=-(u, v)=-e$ in $\mathbb{R}^{E}$.

An $r$-dimensional rational subspace $\Lambda^{\mathbb{R}} \subset \mathbb{R}^{m}$ inherits the inner product $\langle\cdot, \cdot\rangle$. The space $\Lambda^{\mathbb{R}}$ contains two lattices of rank $r$, namely $\Lambda:=\Lambda^{\mathbb{R}} \cap \mathbb{Z}^{m}$ and its dual lattice

$$
\Lambda^{\#}:=\left\{x \in \Lambda^{\mathbb{R}}:\langle x, \lambda\rangle \in \mathbb{Z} \text { for all } \lambda \in \Lambda\right\} .
$$

Since $\langle\Lambda, \Lambda\rangle \subset\left\langle\mathbb{Z}^{m}, \mathbb{Z}^{m}\right\rangle=\mathbb{Z}$, one has an inclusion $\Lambda \subset \Lambda^{\#}$. Their quotient is called the determinant group

$$
\operatorname{det}(\Lambda):=\Lambda^{\#} / \Lambda
$$

Given a rational orthogonal decomposition $\mathbb{R}^{m}=B^{\mathbb{R}} \oplus Z^{\mathbb{R}}$, one obtains two determinant groups $\operatorname{det}(B), \operatorname{det}(Z)$, which turn out to be both isomorphic to what we will call the critical group

$$
K:=\mathbb{Z}^{m} /(B \oplus Z)
$$

of the rational orthogonal decomposition. Indeed, if $\pi_{B}, \pi_{Z}$ denote the orthogonal projections from $\mathbb{R}^{m}$ onto $B^{\mathbb{R}}, Z^{\mathbb{R}}$, then these maps turn out to give rise to surjections from $\mathbb{Z}^{m}$ onto $B^{\#}$ and $Z^{\#}$, respectively, and which induce isomorphisms (see [1, Proposition 3$]$ )

$$
\begin{array}{lcccc}
\operatorname{det}(B) & \cong & K & \cong & \operatorname{det}(Z) \\
B^{\#} / B & \stackrel{\pi_{B}}{\longleftarrow} & \mathbb{Z}^{m} /(B \oplus Z) & \stackrel{\pi_{Z}}{\longrightarrow} & Z^{\#} / Z .
\end{array}
$$

One can compute the critical group $K$ very explicitly as the (integer) cokernel of several matrices, for example via their Smith normal form. If the lattices $B, Z$ have $\mathbb{Z}$-bases $\left\{b_{1}, \ldots, b_{\alpha}\right\},\left\{z_{1}, \ldots, z_{\beta}\right\}$ then let $M_{B}, M_{Z}, M_{B \oplus Z}$ be matrices having columns given by 
$\left\{b_{i}\right\}_{i=1}^{\alpha},\left\{z_{j}\right\}_{j=1}^{\beta},\left\{b_{i}\right\}_{i=1}^{\alpha} \cup\left\{z_{j}\right\}_{j=1}^{\beta}$, respectively. The Gram matrices $M_{B}^{t} M_{B}, M_{Z}^{t} M_{Z}$ express the bases for $B, Z$ in terms of the dual bases for $B^{\#}, Z^{\#}$, and hence

$$
\begin{aligned}
K & \cong \mathbb{Z}^{m} /(B \oplus Z)=\operatorname{coker} M_{B \oplus Z}, \\
& \cong B^{\#} / B=\operatorname{coker}\left(M_{B}^{t} M_{B}\right), \\
& \cong Z^{\#} / Z=\operatorname{coker}\left(M_{Z}^{t} M_{Z}\right) .
\end{aligned}
$$

2.2. Functoriality. Suppose that one has two rational orthogonal decompositions $\mathbb{R}^{m_{i}}=$ $B_{i}^{\mathbb{R}} \oplus Z_{i}^{\mathbb{R}}$ for $i=1,2$, and an $\mathbb{R}$-linear map $f: \mathbb{R}^{m_{1}} \rightarrow \mathbb{R}^{m_{2}}$. When does $f$ induce a homomomorphism $f: K_{1} \rightarrow K_{2}$ between their critical groups?

It is natural to assume that $f$ carries the integer lattice $\mathbb{Z}^{m_{1}}$ into $\mathbb{Z}^{m_{2}}$, that is, $f$ is represented by a matrix in $\mathbb{Z}^{m_{2} \times m_{1}}$. Note that this already implies that the adjoint map $f^{t}: \mathbb{R}^{m_{2}} \rightarrow \mathbb{R}^{m_{1}}$ with respect to the standard inner products will also satisfy $f^{t}\left(\mathbb{Z}^{m_{2}}\right) \subset \mathbb{Z}^{m_{1}}$, since this map is represented by the transposed $\mathbb{Z}^{m_{1} \times m_{2}}$ matrix.

What one needs further to induce homomorphisms of critical groups is that $f\left(B_{1}\right) \subset B_{2}$ and $f\left(Z_{1}\right) \subset Z_{2}$. The following proposition gives a useful reformulation.

Proposition 2.3. For a linear map $f: \mathbb{R}^{m_{1}} \rightarrow \mathbb{R}^{m_{2}}$ satisfying $f\left(\mathbb{Z}^{m_{1}}\right) \subset \mathbb{Z}^{m_{2}}$, one has

$$
f\left(B_{1}\right) \subset B_{2} \Longleftrightarrow f^{t}\left(Z_{2}\right) \subset Z_{1} \Longleftrightarrow f\left(Z_{1}\right) \subset Z_{2}^{\#}
$$

and

$$
f\left(Z_{1}\right) \subset Z_{2} \Longleftrightarrow f^{t}\left(B_{2}\right) \subset B_{1} \Longleftrightarrow f\left(B_{1}\right) \subset B_{2}^{\#} .
$$

Proof. All of the implications follow using the adjointness of $f, f^{t}$ with respect to the pairings on $\mathbb{R}^{m_{1}}, \mathbb{R}^{m_{2}}$, along with the definitions of $B_{i}^{\#}, Z_{i}^{\#}$ and the fact that $B_{i}=Z_{i}^{\perp}$.

When a linear map $f: \mathbb{R}^{m_{1}} \rightarrow \mathbb{R}^{m_{2}}$ satisfies all of the conditions in the previous proposition, we say that $f$ is a morphism of rational orthogonal decompositions. It is clear that $f$ then induces a homomorphism $K_{1} \rightarrow K_{2}$ between the critical groups, denoted here also by $f$.

Note the following property of such maps $f$ for future use.

Proposition 2.4. Any morphism $f: \mathbb{R}^{m_{1}} \rightarrow \mathbb{R}^{m_{2}}$ of rational orthogonal decompositions intertwines the projection maps onto either $B_{i}^{\mathbb{R}}$ or $Z_{i}^{\mathbb{R}}$. That is, the following diagram commutes:

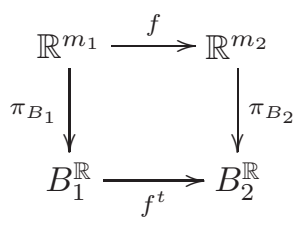

and the same holds replacing $B_{i}$ by $Z_{i}$ everywhere.

Proof. Given $x_{1} \in \mathbb{R}^{m_{1}}$ and $b_{2} \in B_{2}^{\mathbb{R}}$, note that

$$
\left\langle\pi_{B_{2}}\left(f\left(x_{1}\right)\right), b_{2}\right\rangle=\left\langle f\left(x_{1}\right), b_{2}\right\rangle=\left\langle x_{1}, f^{t}\left(b_{2}\right)\right\rangle=\left\langle\pi_{B_{1}}\left(x_{1}\right), f^{t}\left(b_{2}\right)\right\rangle=\left\langle f\left(\pi_{B_{1}}\left(x_{1}\right)\right), b_{2}\right\rangle .
$$

Since this equality holds for any test vector $b_{2} \in B_{2}^{\mathbb{R}}$, one concludes that $\pi_{B_{2}}\left(f\left(x_{1}\right)\right)=$ $f\left(\pi_{B_{1}}\left(x_{1}\right)\right)$. 
2.3. Pontrjagin duality. Every finite abelian group $K$ is isomorphic to its Pontrjagin dual

$$
K^{*}:=\operatorname{Hom}_{\mathbb{Z}}(K, \mathbb{Q} / \mathbb{Z}) .
$$

This isomorphism is not, in general, natural (although the isomorphism $K \cong K^{* *}$ is). However, for critical groups $K=\mathbb{Z}^{m} /(B \oplus Z)$ associated with a rational orthogonal decomposition, the isomorphism comes about naturally from the pairing

$$
\begin{array}{ccc}
\mathbb{Z}^{m} \times \mathbb{Z}^{m} & \rightarrow & \mathbb{Q} \\
(x, y) & \mapsto & \langle\pi(x), \pi(y)\rangle
\end{array}
$$

where $\pi$ is either of the orthogonal projections $\pi_{B}$ or $\pi_{Z}$. This induces a pairing

$$
\langle\cdot, \cdot\rangle: K \times K \rightarrow \mathbb{Q} / \mathbb{Z}
$$

which is nondegenerate in the sense that the following map is an isomorphism:

$$
\begin{array}{ccc}
K & \rightarrow & \operatorname{Hom}_{\mathbb{Z}}(K, \mathbb{Q} / \mathbb{Z}) \quad\left(=K^{*}\right) \\
x & \mapsto & \langle x, \cdot\rangle .
\end{array}
$$

Pontrjagin duality is contravariant in the following sense. Given a homomorphism $f$ : $K_{1} \rightarrow K_{2}$ of abelian groups, there is a dual morphism $f^{*}: K_{2}^{*} \rightarrow K_{1}^{*}$ given by $f^{*}(g)=g \circ f$. The next proposition asserts that this duality interacts as one would expect with morphisms of rational orthogonal decompositions.

Proposition 2.5. For a morphism $f: \mathbb{R}^{m_{1}} \rightarrow \mathbb{R}^{m_{2}}$ of rational orthogonal decompositions, Pontrjagin duality identifies $f^{t}$ with $f^{*}$, in the sense that the following diagram commutes:

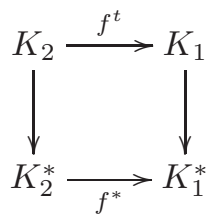

Here the vertical maps are both Pontrjagin duality isomorphisms as in (9).

Proof. Unravelling the definitions, this amounts to checking that if $x_{i} \in \mathbb{Z}^{m_{i}}$ for $i=1,2$, then one has $\left\langle f^{t}\left(x_{2}\right), x_{1}\right\rangle=\left\langle x_{2}, f\left(x_{1}\right)\right\rangle$.

This last proposition has a useful consequence.

Corollary 2.6. For a morphism $f: \mathbb{R}^{m_{1}} \rightarrow \mathbb{R}^{m_{2}}$ of rational orthogonal decompositions, the maps induced by $f, f^{t}$ on critical groups satisfy $\operatorname{ker}(f)^{*} \cong \operatorname{coker}\left(f^{t}\right)$ and $\operatorname{coker}(f)^{*} \cong \operatorname{ker}\left(f^{t}\right)$.

Proof. Pontrjagin duality generally gives $\operatorname{ker}(f)^{*} \cong \operatorname{coker}\left(f^{*}\right)$ and $\operatorname{coker}(f)^{*} \cong \operatorname{ker}\left(f^{*}\right)$, so this follows from Proposition 2.5.

\section{The CRITICAL GROUP OF A GRAPH}

This section particularizes the discussion of critical groups from the previous section to the context of Example 2.2, that is, the critical group $K(G)$ for a graph $G=(V, E)$. It also recalls how one can use spanning trees/forests to be more explicit about some of these constructions, and reviews for later use some other known results about critical groups of graphs.

We will use the term "spanning tree" when discussing connected graphs and "spanning forest" when no connectivity is assumed. 
3.1. Cycles, bonds, Laplacians, and spanning trees. Let $G=(V, E)$ be a graph. After picking an orientation for its edges, the usual cellular boundary map from 1-chains to 0 -chains with real or integer coefficients

$$
\begin{aligned}
& \mathbb{R}^{E} \stackrel{\partial_{G}}{\longrightarrow} \mathbb{R}^{V} \\
& \mathbb{Z}^{E} \stackrel{\partial_{G}}{\longrightarrow} \mathbb{Z}^{V}
\end{aligned}
$$

is defined $\mathbb{R}$ - or $\mathbb{Z}$-linearly as follows: A basis element $e$ corresponding to an edge directed from vertex $u$ to vertex $v$ is sent to $\partial_{G}(e)=+v-u$. One considers the negative $-e$ of this basis element as representing the same edge but directed from $v$ to $u$, which is consistent with

$$
\partial_{G}(-e)=+u-v=-\partial_{G}(e) .
$$

Elements in the kernel of $Z^{\mathbb{R}}:=\operatorname{ker} \partial_{G}$ are called cycles, while elements in the perpendicular space $B^{\mathbb{R}}:=\operatorname{im} \partial_{G}^{t}$ are called bonds. Thus $\mathbb{R}^{E}=B^{\mathbb{R}} \oplus Z^{\mathbb{R}}$ is a rational orthogonal decomposition associated with the graph $G=(V, E)$, and we denote by $K(G)$ the associated critical group.

The lattice $B$ of bonds is known to be spanned by the signed incidence vectors $b\left(V_{1}, V_{2}\right)$ of the directed edges that span across a cut (partition) $V=V_{1} \sqcup V_{2}$. The lattice $Z$ of cycles is known to be spanned by the signed incidence vectors $z(C)$ coming from directed cycles in $G$.

If one wants a smaller $\mathbb{Z}$-spanning set for $B$, one can take the vectors $b_{G}(\{v\}, V \backslash\{v\})$ for cuts that isolate single vertices; this vector $b_{G}(\{v\}, V \backslash\{v\})$ is exactly the row vector of the $|V| \times|E|$ boundary map $\partial_{G}$ indexed by $v$. To simplify notation, we will write

$$
b_{G}(v):=b_{G}(\{v\}, V \backslash\{v\})
$$

for this bond, and we will call it the bond at $v$ in $G$. In order to select out of this spanning set a $\mathbb{Z}$-basis for $B$, one should omit exactly one vertex from each connected component of $G$.

Here are a few consequences of these facts:

(i) The Gram matrix $M_{B}^{t} M_{B}$ corresponding to the above mentioned $\mathbb{Z}$-basis for $B$ gives what is usually called a (reduced) Laplacian matrix $\overline{L(G)}$; the matrix $M_{B}$ is obtained from $\partial_{G}^{t}$ by removing the columns corresponding to the chosen vertex in each connected component of $G$. As a consequence, one has by Kirchhoff's Matrixtree Theorem (see, e.g., [21, Theorem 2.2.12]) that

$$
|K(G)|=\operatorname{det} \overline{L(G)}=\kappa(G),
$$

the number of spanning forests in $G$.

(ii) (The chip-firing/dollar-game/sandpile/Picard presentations for $K(G)$ )

Given a connected graph $G=(V, E)$ with boundary map $\mathbb{Z}^{E} \stackrel{\partial}{\longrightarrow} \mathbb{Z}^{V}$, bond lattice $B:=\operatorname{im} \partial^{t}$, and any vertex $v_{0}$ in $V$, one has an isomorphism

$$
\begin{aligned}
K(G) & \cong \operatorname{coker}\left(M_{B}^{t} M_{B}\right) \\
& \cong \operatorname{coker}(\overline{L(G)}) \\
& \cong \mathbb{Z}^{V \backslash\left\{v_{0}\right\}} / \overline{L(G)} \\
& \cong \mathbb{Z}^{V} /\left(\mathbb{Z} v_{0}+\operatorname{im}\left(\partial_{G} \partial_{G}^{t}\right)\right) \\
& \cong \mathbb{Z}^{V} /\left(\mathbb{Z} v_{0}+\partial_{G}(B)\right) \\
& \cong \mathbb{Z}^{V} /\left(\mathbb{Z} v_{0}+\mathbb{Z}\left(\partial_{G} b_{G}(v)_{v \in V}\right)\right) .
\end{aligned}
$$



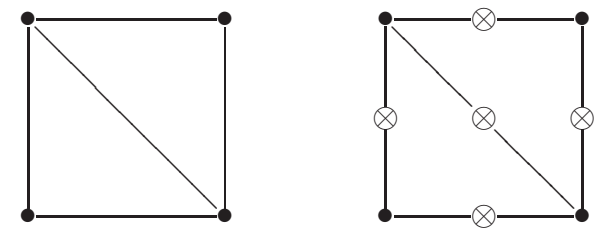

Figure 2. A graph $G$ and its edge subdivision sd $G$.

(iii) For any vertex $u$ of $G$, one has the relation

$$
\sum_{\{v \in V:\{u, v\} \in E\}}(u, v)=0
$$

in $K(G)=\mathbb{Z}^{E} /(B \oplus Z)$.

(iv) For any directed cycle $u_{0} \rightarrow u_{1} \rightarrow \cdots \rightarrow u_{\ell-1} \rightarrow u_{\ell}=u_{0}$ in $G$, one has the relation

$$
\sum_{i=0}^{\ell-1}\left(u_{i}, u_{i+1}\right)=0
$$

in $K(G)=\mathbb{Z}^{E} /(B \oplus Z)$.

Fixing a particular spanning forest $T$ for $G$ allows one to simultaneously construct $\mathbb{Z}$ bases of $B$ and $Z$. Removing any edge $e$ in the forest $T$ creates a new connected component in the forest, say with vertex set $V_{e} \subset V$; ranging over all edges $e$ in $T$, the signed incidence vectors $b_{e}^{T}$ for the cuts $V=V_{e} \sqcup\left(V-V_{e}\right)$ form a $\mathbb{Z}$-basis for $B$. Dually, adding any edge $e$ in $E-T$ to $T$ creates a unique cycle in $T \cup\{e\}$; ranging over all edges in $E-T$, the signed incidence vectors $z_{e}^{T}$ of these cycles form a $\mathbb{Z}$-basis for $Z$.

3.2. A presentation for $K($ line $G$ ). Proposition 3.1 below gives a useful presentation for $K$ (line $G$ ) that is an immediate consequence of the last equation in assertion (3.1)(ii) above. It will be used both in the proof of Theorem 1.1 and in the analysis of $K$ (line $K_{n}$ ) in Section 9.1.

Let $G=(V, E)$ be a connected simple graph, so that line $G=\left(V_{\text {line } G}, E_{\text {line } G}\right)$ is also connected. Identify the vertex set $V_{\text {line }} G$ of the line graph of $G$ with the edge set $E$ of $G$. After picking arbitrary orientations for the edges of line $G$, consider the boundary map for line $G$ :

$$
\partial_{\text {line } G}: \mathbb{Z}^{E_{\text {line } G} \longrightarrow \mathbb{Z}^{E}}\left(=\mathbb{Z}^{\left.V_{\text {line } G}\right)} .\right.
$$

Proposition 3.1. Given a connected simple graph $G=(V, E)$ and any edge $e_{0}$ in $E$, one has an isomorphism

$$
\begin{aligned}
K(\text { line } G) & \cong \mathbb{Z}^{E} /\left(\mathbb{Z} e_{0}+\partial_{\text {line } G}\left(B_{\text {line } G}\right)\right) \\
& \cong \mathbb{Z}^{E} /\left(\mathbb{Z} e_{0}+\mathbb{Z}\left(\partial_{\text {line } G} b_{\text {line } G}(e)_{e \in E}\right)\right) .
\end{aligned}
$$

3.3. Lorenzini's result on edge subdivisions. The edge subdivision of a graph $G$ is the graph sd $G$ obtained by creating a new midpoint vertex called $u v$ for every edge $\{u, v\}$ of $G$; that is, $\{u, v\}$ is removed and replaced by two edges $\{u, u v\},\{v, u v\}$ in $\operatorname{sd} G$. An orientation of $G$ induces an orientation of $\operatorname{sd} G$ : If $u$ is oriented towards $v$ then $u$ is oriented towards $u v$ and $u v$ is oriented towards $v$.

In [13] Lorenzini first observed that the critical groups $K(\operatorname{sd} G)$ and $K(G)$ determine each other in a trivial way, using the description $K=Z^{\#} / Z$ as we now explain. If $\left\{C_{1}, \ldots, C_{\beta}\right\}$ 
is any set of directed cycles in $G$ whose incidence vectors $\left\{z\left(C_{i}\right)\right\}_{i=1}^{\beta}$ give a $\mathbb{Z}$-basis for $Z_{G}$, then one can subdivide those same cycles to obtain a $\mathbb{Z}$-basis $\left\{z_{\mathrm{sd} C_{i}}\right\}_{i=1}^{\beta}$ for $Z_{\mathrm{sd} G}$. One then checks that

$$
\left\langle z_{\mathrm{sd} C_{i}}, z_{\mathrm{sd} C_{j}}\right\rangle=2\left\langle z\left(C_{i}\right), z\left(C_{j}\right)\right\rangle,
$$

for each $i, j$, since the inner product counts (with signs) the overlap of edges between cycles $C_{i}, C_{j}$, and these overlaps double in size after the subdivision. Hence one has the following relation between their Gram matrices:

$$
M_{\mathrm{sd} G}^{t} M_{\mathrm{sd} G}=2 M_{G}^{t} M_{G}
$$

and the following simple relation between their cokernels, the critical groups:

Proposition 3.2 (Lorenzini [13]). Let $G$ be a graph with $\beta$ independent cycles. Expressing $K(G) \cong \bigoplus_{i=1}^{\beta} \mathbb{Z}_{d_{i}}$ for positive integers $d_{1}, d_{2}, \ldots, d_{\beta} \geq 1$, one has $K(\operatorname{sd} G) \cong \bigoplus_{i=1}^{\beta} \mathbb{Z}_{2 d_{i}}$.

It will be useful later to have an expression of this result in terms of explicit morphisms (as was done also in [13]). Consider the pair of adjoint maps defined $\mathbb{R}$-linearly by

$$
\begin{array}{ccc}
\mathbb{R}^{E_{\text {sd } G}} & \longmapsto & \mathbb{R}^{E_{G}} \\
(u, u v) & \longmapsto & (u, v) \\
(u v, v) & \longmapsto & (u, v) \\
& & \\
\mathbb{R}^{E_{G}} & \stackrel{h^{t}}{\longmapsto} & \mathbb{R}^{E_{\text {sd } G}} \\
(u, v) & \longmapsto & (u, u v)+(u v, v) .
\end{array}
$$

One can easily check that these are morphisms of rational orthogonal decompositions, and hence give rise to a morphism $h: K(\operatorname{sd} G) \rightarrow K(G)$ of critical groups. The relation (11) between the two $\beta \times \beta$ Gram matrices shows that the kernel-cokernel exact sequence associated to $h$ takes this form:

$$
\begin{aligned}
& 0 \longrightarrow \operatorname{ker}(h) \quad \longrightarrow \quad K(\operatorname{sd} G) \quad \stackrel{h}{\longrightarrow} \quad K(G) \quad \longrightarrow 0 \\
& 0 \longrightarrow \mathbb{Z}_{2}^{\beta} \longrightarrow \bigoplus_{i=1}^{\beta} \mathbb{Z}_{2 d_{i}} \longrightarrow \bigoplus_{i=1}^{\beta} \mathbb{Z}_{d_{i}} \longrightarrow 0 \text {. }
\end{aligned}
$$

Proposition 3.2 is equivalent to the assertion that $K(\operatorname{sd} G)$ can be generated by $\beta$ elements and fits into an exact sequence of this form, generalizing equation (4) from the Introduction.

3.4. A non-standard treatment of the complete graph. Let $K_{n}$ be the complete graph on $n$ vertices. A celebrated formula of Cayley asserts that $\kappa\left(K_{n}\right)=n^{n-2}$ (see, e.g., [8, Section 13.2]). Generalizing this to compute the critical group $K\left(K_{n}\right)$ is a favorite example of many papers in the subject. We approach this calculation in a slightly nonstandard way here, mainly because it will provide us with a crucial technical lemma for later use in Section 6.4.

Proposition 3.3. The complete graph $K_{n}$ has critical group

$$
K\left(K_{n}\right) \cong \mathbb{Z}_{n}^{n-2} \text {. }
$$

Furthermore, in the presentation $K\left(K_{n}\right)=\mathbb{Z}^{E} /(B \oplus Z)$, a minimal generating set is provided by the images of any set of $n-2$ edges which form a spanning tree connecting $n-1$ out of the $n$ vertices.

Proof. Since Cayley's formula implies $\left|K\left(K_{n}\right)\right|=\left|\mathbb{Z}_{n}^{n-2}\right|$, it will suffice to show that $K\left(K_{n}\right)$ is all $n$-torsion and that it can be generated by $n-2$ elements as in the second assertion. Let $[n]:=\{1,2, \ldots, n\}$ denote the vertex set $V$ for $K_{n}$. 
To show $K\left(K_{n}\right)$ is all $n$-torsion, given any directed edge $e=(i, j)$ in $K_{n}$, we will prove that $n \cdot e$ is equal to a sum of cycles and bonds. Indeed, we can take the sum of the directed cycles $(i, j)+(j, k)+(k, i)$ for $k \in[n]-\{i, j\}$, and add the two bonds

$$
\begin{aligned}
b(\{i\},[n]-\{i\}) & =(i, 1)+(i, 2)+\cdots+(i, n) \\
b([n]-\{j\},\{j\}) & =(1, j)+(2, j)+\cdots+(n, j) .
\end{aligned}
$$

For the second assertion, let $T$ be a collection of $n-2$ edges that form a spanning tree connecting $n-1$ out of the $n$ vertices. By symmetry, we may assume that $n$ is the vertex that is isolated by $T$. The edges of $K_{n}$ can be partitioned into two sets, $E\left(K_{n-1}\right)$ and $\{(i, n)\}_{i=1}^{n-1}$.

Any edge $e$ in $E\left(K_{n-1}\right)$ either lies in $T$ or $T \cup\{e\}$ contains a unique cycle that lets one express $e$ in terms of the elements of $T$ modulo $Z\left(K_{n-1}\right)$, and hence modulo $Z=Z\left(K_{n}\right)$.

For each $1 \leq i \leq n-1$, the bond

$$
b_{i}:=\sum_{\substack{j=1 \\ j \neq i}}^{n}(i, j) \equiv 0 \quad \bmod B,
$$

and it follows that

$$
(i, n) \equiv-\sum_{\substack{j=1 \\ j \neq i}}^{n-1}(i, j) \quad \bmod B .
$$

The edges in the sum all belong to $K_{n-1}$ and thus, according to the previous paragraph, can be written in terms of $T$ modulo $Z$. It follows that $(i, n)$ can be written in terms of $T$ modulo $B+Z$.

\section{Proof of Theorem 1.1}

Recall the statement of Theorem 1.1:

Theorem 1.1. When the simple graph $G$ is 2-edge-connected, the critical group $K$ (line $G$ ) can be generated by $\beta(G)$ elements.

The $\beta(G)$ generators will come from the set of edges in the complement $E \backslash T$ of a carefully chosen spanning tree $T$ for $G$. For this we introduce the following technical condition, which we have not encountered elsewhere.

Definition 4.1. For a connected graph $G=(V, E)$, say that a spanning tree $T \subset E$ for $G$ has an absorption order in $G$ if one can linearly order the union $V \sqcup T$ of its vertices and edges in the following way:

(i) The order begins with a vertex $v_{0}$ in $V$ followed by an edge $e_{0}$ of $T$, such that $e_{0}$ is the unique edge of $T$ incident to $v_{0}$ (so $v_{0}$ is a leaf-vertex of $T$ attached along the leaf-edge $e_{0}$ ).

(ii) For every other vertex $v$ in $V \backslash\left\{v_{0}\right\}$, there exists an edge $e=\{v, w\}$ such that $w$ occurs earlier in the order than $v$, and the edge $e$ either lies in $E \backslash T$ or occurs earlier in the order than $v$.

(iii) For every other edge $e$ in $T \backslash\left\{e_{0}\right\}$, there exists a vertex $v$ incident to $e$ which occurs earlier in the order than $e$, and every other edge incident to $v$ either lies in $E \backslash T$ or occurs earlier in the order than $e$. 
The relevance of an absorption order for a spanning tree is given by the algebraic consequence in the following proposition. Say that an orientation of the edges of a tree $T$ is bipartite if, for every vertex $v$, the edges of $T$ incident to $v$ are either all oriented toward $v$ or all oriented away from $v$.

Proposition 4.2. Let $G=(V, E)$ be a simple graph, and assume it has a spanning tree $T \subset E$ which has an absorption order in $G$.

Then the images of the basis elements in $\mathbb{Z}^{E}$ corresponding to the edges $E \backslash T$ not lying on $T$ give a set of $\beta(G)$ generators for $K($ line $G)$, using the presentation from Proposition 3.1

$$
K(\text { line } G) \cong \mathbb{Z}^{E} /\left(\mathbb{Z} e_{0}+\partial_{\text {line } G}\left(B_{\text {line } G}\right)\right)
$$

assuming that the orientation chosen for $G$ restricts to a bipartite orientation of $T$ (although line $G$ may be oriented arbitrarily), and the edge $e_{0}$ is the designated leaf-edge of $T$ appearing second in the absorption order.

To prove this, note the following crucial lemma:

Lemma 4.3. When a connected graph $G=(V, E)$ is oriented in a way that restricts to a bipartite orientation for a spanning tree $T \subset E$, then any edge $e=\{v, w\}$ has

$$
b_{G}(v) \equiv \pm b_{G}(w) \quad \bmod \quad \mathbb{Z} e+\mathbb{Z}(E \backslash T)+\partial_{\text {line } G}\left(B_{\text {line } G}\right) .
$$

Proof of Lemma 4.3. Label the edges of $G$ incident to $v$ other than $e$ by

$$
\underbrace{e_{1}, \ldots, e_{p}}_{\text {in } T}, \underbrace{e_{p+1}, e_{p+2}, \ldots, e_{P}}_{\text {in } E \backslash T},
$$

and those incident to $w$ other than $e$ by

$$
\underbrace{f_{1}, \ldots, f_{q}}_{\text {in } T}, \underbrace{f_{q+1}, f_{q+2}, \ldots, f_{Q}}_{\text {in } E \backslash T} .
$$

With these notations, one then has

$$
\begin{aligned}
\partial_{\text {line } G} b_{\text {line } G}(e) & =\left(e_{1}-e\right)+\cdots+\left(e_{P}-e\right)+\left(f_{1}-e\right)+\cdots+\left(f_{Q}-e\right) \\
& =\left(e_{1}+\cdots+e_{P}\right)+\left(f_{1}+\cdots+f_{Q}\right)-(P+Q) e
\end{aligned}
$$

Because the orientation of $G$ when restricted to $T$ is bipartite,

$$
\begin{aligned}
b_{G}(v) & = \pm\left(e_{1}+\cdots+e_{p}\right) \pm e_{p+1} \pm e_{p+2} \pm \cdots \pm e_{P} \\
b_{G}(w) & = \pm\left(f_{1}+\cdots+f_{q}\right) \pm f_{q+1} \pm f_{q+2} \pm \cdots \pm f_{Q} .
\end{aligned}
$$

Comparison of (12) and (13) shows that one of the two expressions $b_{G}(v)+b_{G}(w)$ or $b_{G}(v)-$ $b_{G}(w)$ differs from $\partial_{\text {line } G} b_{\text {line } G}(e)$ by a $\mathbb{Z}$-linear combination of the edges in

$$
\{e\} \cup\left\{e_{p+1}, e_{p+2}, \cdots, e_{P}, f_{q+1}, f_{q+2}, \cdots, f_{Q}\right\} .
$$

Since the second set in the above union lies in $E \backslash T$, the lemma follows.

Proof of Proposition 4.2. One needs to show that the subgroup of $\mathbb{Z}^{E}$ defined by

$$
I:=\mathbb{Z}(E \backslash T)+\mathbb{Z} e_{0}+\partial_{\text {line } G}\left(B_{\text {line } G}\right)
$$

is all of $\mathbb{Z}^{E}$. Since $E \backslash T$ is a subset of $I$, it is enough to show that every edge $e$ in $T$ lies in $I$. More strongly, one shows by induction on their location in the absorption order for $T$, that not only does every edge $e$ in $T$ lie in $I$, but also every vertex $v$ in $V$ has $b_{G}(v)$ lying in $I$. 

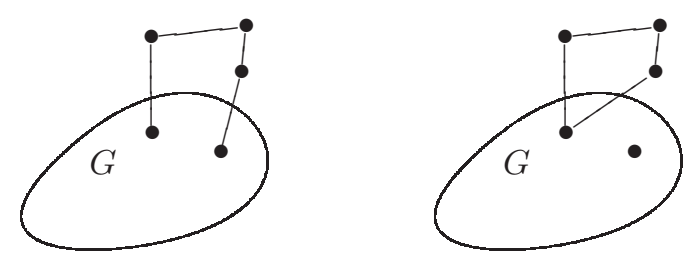

FiguRE 3. Graphs with open and closed ears.

The base case for this induction deals both with the first vertex $v_{0}$ and the first edge $e_{0}$, which come at the beginning of the absorption order. Since $v_{0}$ is a leaf vertex of $T$ along the edge $e_{0}$, one has $b_{G}\left(v_{0}\right)$ in $I$, since the only edges incident to $v_{0}$ are $e_{0}$ and edges of $E \backslash T$. For the edge $e_{0}$, note that it trivially lies in $I$.

In the inductive step, the next element in the absorption order is either a vertex $v \neq v_{0}$ or an edge $e \neq e_{0}$.

If the next element is a vertex $v \neq v_{0}$, then by Definition 4.1(ii), there exists an edge $e=\{v, w\}$ for which $b_{G}(w)$ lies in $I$ by induction, and either $e$ lies in $E \backslash T$ (so that $e$ is in $I$ ) or $e$ is earlier in the order than $v$ (so that $e$ is in $I$ by induction). Hence Lemma 4.3 shows that $b_{G}(v)$ also lies in $I$.

If the next element is an edge $e \neq e_{0}$, then by Definition 4.1(iii), there exists a vertex $v$ incident to $e$ for which $b_{G}(v)$ lies in $I$ by induction. Note that $b_{G}(v)$ is a \pm 1 combination of all the edges $e^{\prime}$ incident to $v$, and all of these other edges $e^{\prime} \neq e$ either have $e^{\prime}$ in $E \backslash T$ (so that $e^{\prime}$ is in $I$ ) or $e^{\prime}$ is earlier in the order than $e$ (so that $e^{\prime}$ is in $I$ by induction). Hence $e$ also lies in $I$.

To show that 2-edge-connected graphs $G$ always have a spanning tree $T$ with an absorption order, we recall the well-known reformulation of 2-edge-connectivity in terms of ear decompositions; see e.g., [21, Definition 4.2.7].

Definition 4.4. Let $G=(V, E)$ be a simple graph. An ear of $G$ is a walk that alternates (incident) vertices $u_{i}$ and edges $e_{i}$

$$
v:=u_{1}, e_{1}, u_{2}, e_{2}, \ldots, u_{\ell}, e_{\ell}, u_{\ell+1}:=w
$$

such that the internal vertices $u_{2}, \ldots, u_{\ell}$ are each of degree 2 in $G$. If $v \neq w$, it is called an open ear (and necessarily $\ell \geq 1$ ), while if $v=w$, it is called a closed ear (and necessarily $\ell \geq 3$, because $G$ is simple).

An ear decomposition of $G$ is a decomposition of its vertices and edges

$$
P_{0} \cup P_{1} \cup \cdots \cup P_{k}
$$

such that $P_{0}$ is a cycle, and for $1 \leq i \leq k, P_{i}$ is an ear of $P_{0} \cup P_{1} \cup \cdots \cup P_{i}$.

Proposition 4.5 ([21, Theorem 4.2.8]). A graph is 2-edge-connected if and only if it has an ear decomposition.

In light of Proposition 4.2, the following result implies Theorem 1.1.

Proposition 4.6. Let $G=(V, E)$ be a simple 2-edge-connected graph. Then $G$ has at least one spanning tree $T \subset E$ with an absorption order in $G$. 
Proof. Induct on the number $k$ of ears in an ear decomposition $P_{0} \cup P_{1} \cup \cdots \cup P_{k}$ for $G$.

In the base case $k=0$, the graph $G=P_{0}$ is a $n$-cycle. Label its vertices $V=$ $\left\{v_{0}, v_{1}, \ldots, v_{n-1}\right\}$ and edges $E=\left\{e_{0}, e_{1}, \ldots, e_{n-1}\right\}$ so that $e_{i}=\left\{v_{i}, v_{i+1}\right\}$ with indices taken modulo $n$. Then one can easily check that $T=\left\{e_{0}, e_{1}, \ldots, e_{n-2}\right\}$ is a spanning tree, and $\left(v_{0}, e_{0}, v_{1}, e_{1}, \ldots, v_{n-2}, e_{n-2}, v_{n-1}\right)$ is an absorption order for $T$ in $G$.

In the inductive step, one may assume that $G^{-}:=P_{0} \cup P_{1} \cup \cdots \cup P_{k-1}$ has a spanning tree $T^{-}$with an absorption order in $G^{-}$. Choose the labelling of the endpoints $v, w$ of the ear $P_{k}$ so that $v$ comes weakly earlier than $w$ in the absorption order for $T^{-}$, where the vertices and edges of $P_{k}$ are labelled as in (14). Extend $T^{-}$to

$$
T:=T^{-} \sqcup\left\{u_{2}, u_{3}, \ldots, u_{\ell}\right\}
$$

which is easily seen to be a spanning tree for $G$. One extends the absorption order for $T^{-}$ in $G^{-}$to one for $T$ in $G$ by inserting the subsequence

$$
\left(u_{2}, e_{2}, u_{3}, e_{3}, u_{4}, \ldots, u_{\ell}, e_{\ell}\right)
$$

into the absorption sequence for $T^{-}$in one of two possible locations, depending upon whether $v$ and $w$ are the initial vertex $v_{0}$ of the absorption order of $T^{-}$, or not.

First we assume that $P_{k}$ is an open ear (that is, $v \neq w$ ) or $P_{k}$ is a closed ear with $v=w \neq v_{0}$. In this case, one can check that inserting the subsequence (15) immediately after $v$ in the absorption order for $T^{-}$in $G^{-}$gives an absorption order for $T$ in $G$.

In the case that $P_{k}$ is a closed ear with $v=w=v_{0}$, one checks that inserting the subsequence (15) at the very beginning of the absorption order for $T^{-}$in $G^{-}$gives an absorption order for $T$ in $G$. Note that $u_{2}, e_{2}$ become the "new" $v_{0}, e_{0}$ in this absorption order.

We remark that the converse of Proposition 4.6 is false. For example, one can check that the simple graph $G$ on vertex set $V=\{1,2,3,4\}$ with edges $\{12,13,23,34\}$, which is not 2-edge-connected, does have an absorption ordering for any choice of a spanning tree $T$ in $G$. We have not investigated extensively the problem of characterizing which graphs $G$ contain a spanning tree $T$ with an absorption ordering.

\section{Proof of Theorem 1.2}

For a prime $p$ let $k(p)$ be the largest integer such that $p^{k(p)}$ divides all of the sums $\operatorname{deg}_{G}(v)+\operatorname{deg}_{G}(w)$ as one runs through all edges $e=\{v, w\}$ in the edge set $E$ of $G$. The goal of this section is to give a proof of Theorem 1.2, which we now recall.

Theorem 1.2. Let $G=(V, E)$ be a connected simple graph that contains at least one cycle of even length. Use the abbreviated notation $K:=K($ line $G)$, and let $p$ be a prime for which the quantity $k(p) \geq 1$.

Then for $G$ bipartite, one has

$$
K / p^{k(p)} K \cong \mathbb{Z}_{p^{k(p)}}^{\beta(G)-1} \oplus \mathbb{Z}_{\operatorname{gcd}\left(p^{k},|V|\right)},
$$

while for $G$ nonbipartite, one has

$$
K / p^{k(p)} K \cong \mathbb{Z}_{p^{k(p)}}^{\beta(G)-2} \oplus \begin{cases}0 & \text { if } p \text { is odd, } \\ \mathbb{Z}_{2}^{2} & \text { if } p=2 \text { and }|V| \text { is even, } \\ \mathbb{Z}_{4} & \text { if } p=2 \text { and }|V| \text { is odd. }\end{cases}
$$


Proof. One works again with the presentation from Proposition 3.1

$$
K:=K(\text { line } G)=\mathbb{Z}^{E} /\left(\mathbb{Z} e_{0}+\mathbb{Z}\left(\partial_{\text {line } G} b_{\text {line } G}(e)\right)_{e \in E}\right)
$$

for some choice of an edge $e_{0}$ in $E$. Given a vertex $v$ in $V$, let $\delta_{G}(v)$ denote the element of $\mathbb{Z}^{E}$ which is the sum with coefficient +1 of the basis elements in $\mathbb{Z}^{E}$ corresponding to edges incident with $v$. Given any edge $e=\{v, w\}$ in $E$, reasoning as in equation (12), one finds that

$$
\partial_{\text {line } G} b_{\text {line } G}(e)=\delta_{G}(v)+\delta_{G}(w)-\left(\operatorname{deg}_{G}(v)+\operatorname{deg}_{G}(w)\right) e .
$$

Letting $q:=p^{k(p)}$, one has therefore in $K / q K$ the relation

$$
\partial_{\text {line } G} b_{\text {line } G}(e) \equiv \delta_{G}(v)+\delta_{G}(w)
$$

and one can write a presentation for $K / q K$ as

$$
K / q K=\mathbb{Z}_{q}^{E} /\left(\mathbb{Z}_{q} e_{0}+\mathbb{Z}_{q}\left(\delta_{G}(v)+\delta_{G}(w)\right)_{e=\{v, w\} \in E}\right) .
$$

We now make a particular choice of the edge $e_{0}$ for this presentation, and exhibit a subset of $E$ having size $\beta(G)-2$ or $\beta(G)-1$ which will represent $\mathbb{Z}_{q}$-linearly independent elements in $K / q K$. Because $G$ contains an even-length (not necessarily minimal) cycle, it is possible to choose an edge $e_{0}$ in $E$ which lies on a minimal cycle, so that $E \backslash\left\{e_{0}\right\}$ still connects all of $V$, and so that $E \backslash\left\{e_{0}\right\}$ contains at least one odd cycle in the case where $G$ is nonbipartite. Now, in the bipartite case, pick $S \subset E \backslash\left\{e_{0}\right\}$ to be minimal with respect to the property that $S$ connects all of $V$. In the non-bipartite case, pick $S$ to be minimal with respect to the following three properties: first, $S$ must connect all of $V$; second, $S$ must contain a unique cycle; and third, this cycle must be of odd length. This means that when $G$ is bipartite, $S$ is a spanning tree that avoids $e_{0}$, and when $G$ is nonbipartite, $S$ is a unicyclic spanning subgraph that avoids $e_{0}$, whose unique cycle $C$ is of odd length.

We first wish to show that, in either case, the images of the elements $E \backslash S \backslash\left\{e_{0}\right\}$ are $\mathbb{Z}_{q^{-}}$ linearly independent in the presentation (16); note that this set $E \backslash S \backslash\left\{e_{0}\right\}$ has cardinality $\beta(G)-1$ when $G$ is bipartite, and cardinality $\beta(G)-2$ when $G$ is nonbipartite.

So assume that $E \backslash S \backslash\left\{e_{0}\right\}$ are $\mathbb{Z}_{q^{-}}$-linearly dependent in $K / q K$. Grouping the $\mathbb{Z}_{q^{-}}$ coefficients $c_{v}$ in front of each $\delta_{G}(v)$, one would have a sum $\sum_{v \in V} c_{v} \delta_{G}(v)$ lying in $\mathbb{Z}_{q} e_{0}+$ $\mathbb{Z}_{q}\left(E \backslash S \backslash\left\{e_{0}\right\}\right)$. Thus this sum should have zero coefficient on every edge $e=\{v, w\}$ in $S$, implying that $c_{v}=-c_{w}$ for every such edge. Because $S$ is a spanning set of edges, this forces the existence of a constant $c$ in $\mathbb{Z}_{q}$ for which every $v$ in $V$ has $c_{v}= \pm c$. In fact, when $G$ is bipartite with vertex bipartition $V=V_{1} \sqcup V_{2}$, this forces $c_{v_{1}}=c=-c_{v_{2}}$ for all $v_{1}$ in $V_{1}$ and $v_{2}$ in $V_{2}$, while for $G$ nonbipartite, the existence of the odd cycle $C$ inside $S$ forces $c_{v}=c=-c$ for all $v$ in $V$. In either case, this means that $c_{v}=-c_{w}$ for all edges $e=\{v, w\}$ in $E$, and hence the sum $\sum_{v \in V} c_{v} \delta_{G}(v)$ is actually zero in $\mathbb{Z}_{q}^{E}$. Thus the linear independence is trivial.

It only remains now to analyze the quotient

$$
\frac{K / q K}{\mathbb{Z}_{q}\left(E \backslash S \backslash\left\{e_{0}\right\}\right)}=\mathbb{Z}_{q}^{E} /\left(\mathbb{Z}_{q}(E \backslash S)+\mathbb{Z}_{q}\left(\delta_{G}(v)+\delta_{G}(w)\right)_{e=\{v, w\} \in E}\right) .
$$

Note that when $m$ is odd, for any sequence of vertices $v_{0}, v_{1}, \ldots, v_{m-1}, v_{m}$ one has a telescoping alternating sum

$$
\sum_{i=0}^{m-1}(-1)^{i}\left(\delta_{G}\left(v_{i}\right)+\delta_{G}\left(v_{i+1}\right)\right)=\delta_{G}\left(v_{0}\right)+\delta_{G}\left(v_{m}\right) .
$$

Also note that $S$ will contain paths of edges of odd length between

- every pair $\left(v_{1}, v_{2}\right)$ in $V_{1} \times V_{2}$ when $G$ is bipartite, and 
- every ordered pair $(v, w)$ in $V \times V$ when $G$ is nonbipartite.

Thus, in either case, one has

$$
\mathbb{Z}_{q}\left(\delta_{G}(v)+\delta_{G}(w)\right)_{e=\{v, w\} \in E}=\mathbb{Z}_{q}\left(\delta_{G}(v)+\delta_{G}(w)\right)_{e=\{v, w\} \in S} .
$$

Using this last equation, one can rewrite the quotient on the right of (17) as

$$
\mathbb{Z}_{q}^{S} / \mathbb{Z}_{q}\left(\delta_{S}(v)+\delta_{S}(w)\right)_{e=\{v, w\} \in S}
$$

where here we regard $S$ itself as a graph, namely the edge-induced subgraph of $G=(V, E)$ having the same vertex set $V$ and edge set $S \subset E$.

Note that this last expression in (18) does not depend upon the ambient graph $G$, but only on the subgraph $S$. We therefore rename it $K_{q}(S)$ to emphasize this dependence on $S$ alone. It remains to analyze this group $K_{q}(S)$ in both the bipartite and nonbipartite cases. Case 1: $G$ is bipartite (and hence so is $S$ ). It follows that $S$ is a spanning tree on $V$, with vertex bipartition $V=V_{1} \sqcup V_{2}$. By the above discussion,

$$
\begin{aligned}
K_{q}(S) & =\mathbb{Z}_{q}^{S} / \mathbb{Z}_{q}\left(\delta_{S}(v)+\delta_{S}(w)\right)_{\left(v_{1}, v_{2}\right) \in V_{1} \times V_{2}} \\
& =\mathbb{Z}_{q}^{S} / \mathbb{Z}_{q}\left(\sum_{v \in V} c_{v} \delta_{S}(v): \sum_{v_{1} \in V_{1}} c_{v_{1}}=\sum_{v_{2} \in V_{2}} c_{v_{2}} \text { in } \mathbb{Z}_{q}\right) .
\end{aligned}
$$

We first show by induction on $|S|$ that $K_{q}(S)$ is cyclic, generated by the image of any leaf edge $e$ of $S$, that is, an edge $e$ incident to some leaf vertex $v$ having $\operatorname{deg}_{S}(v)=1$. The base case $|S|=1$ is trivial. In the inductive step, pick another leaf edge $e^{\prime}$ in $S$; we will show it has image 0 in the quotient $K_{q}(S) / \mathbb{Z}_{q} e$. If $e^{\prime}$ is incident to leaf vertex $v^{\prime}$, then for any $c$ in $\mathbb{Z}_{q}$, one has

$$
e^{\prime}+c e=\delta_{S}\left(v^{\prime}\right)+c \delta_{S}(v) .
$$

Taking $c=-1$ (respectively +1 ) when $v, v^{\prime}$ lie in the same (resp. different) set $V_{1}$ or $V_{2}$, one obtains an element that is zero in $K_{q}(S)$, and hence $e^{\prime} \equiv 0$ in $K_{q}(S) / \mathbb{Z}_{q} e$. Now, replacing $S$ by $S \backslash\left\{e^{\prime}\right\}$, one can induct on $|S|$, completing the inductive step and showing that $K_{q}(S)$ is generated by $e$.

We next analyze the order of this cyclic generator $e$ within $K_{q}(S)$. We claim that $c \cdot e=0$ in $K_{q}(S)$ if and only if $c$ lies in $|V| \mathbb{Z}_{q}$. This would finish the proof in the bipartite case, as it would show that $K_{q}(S)$ is isomorphic to the subgroup of $\mathbb{Z}_{q}$ generated by the element $|V|$. This subgroup is isomorphic to $\mathbb{Z}_{\operatorname{gcd}(q,|V|)}$, where $q=p^{k}$. Hence this would imply $K / q K \cong \mathbb{Z}_{p^{k(p)}}^{\beta(G)-1} \oplus \mathbb{Z}_{\operatorname{gcd}\left(p^{k(p)},|V|\right)}$, as desired.

To see the claim, assume that $c \cdot e=0$ in $K_{q}(S)$ for some $c$ in $\mathbb{Z}_{q}$. This means one has a $\operatorname{sum} \sum_{v \in V} c_{v} \delta_{G}(v)=c \cdot e$ in which $\sum_{v_{1} \in V_{1}} c_{v_{1}}=\sum_{v_{2} \in V_{2}} c_{v_{2}}$. This happens if and only if the sum has zero coefficient on all edges $e^{\prime}$ in $S \backslash\{e\}$. If $e=\{v, w\}$ with the leaf vertex $v$ lying in $V_{1}$, and $w$ in $V_{2}$, this means $c_{v_{2}}=c_{w}=-c_{v_{1}}$ for all $v_{1} \in V_{1} \backslash\{v\}$ and $v_{2} \in V_{2} \backslash\{w\}$. Then the condition $\sum_{v_{1} \in V_{1}} c_{v_{1}}=\sum_{v_{2} \in V_{2}} c_{v_{2}}$ forces

$$
c_{v}+\left(\left|V_{1}\right|-1\right)\left(-c_{w}\right)=\left|V_{2}\right|\left(c_{w}\right)
$$

i.e., $c_{v}=(|V|-1) c_{w}$. Hence this can occur if and only if $c=c_{v}+c_{w}=|V| c_{w}$, that is, if $c$ lies in $|V| \mathbb{Z}_{q}$. 
Case 2. $G$ is nonbipartite (and hence so is $S$ ). In this case $S$ is a spanning unicyclic graph, whose unique cycle $C$ is of odd length. By the above discussion,

$$
\begin{aligned}
K_{q}(S) & =\mathbb{Z}_{q}^{S} / \mathbb{Z}_{q}\left(\delta_{S}(v)+\delta_{S}(w)\right)_{(v, w) \in V \times V} \\
& =\mathbb{Z}_{q}^{S} /\left\{\sum_{v \in V} c_{v} \delta_{S}(v): \sum_{v \in V} c_{v} \in 2 \mathbb{Z}_{q}\right\} .
\end{aligned}
$$

Thus, if one defines the tower of $\mathbb{Z}$-lattices (i.e., free abelian groups)

$$
L:=\mathbb{Z}^{S} \supset M:=\mathbb{Z}\left(\delta_{S}(v)\right)_{v \in V} \supset N:=\left\{\sum_{v \in V} c_{v} \delta_{S}(v): \sum_{v \in V} c_{v} \in 2 \mathbb{Z}\right\},
$$

then one has a short exact sequence

$$
0 \rightarrow \underbrace{\frac{M \otimes_{\mathbb{Z}} \mathbb{Z}_{q}}{N \otimes_{\mathbb{Z}} \mathbb{Z}_{q}}}_{(M / N) \otimes_{\mathbb{Z}} \mathbb{Z}_{q}} \rightarrow \underbrace{\frac{L \otimes_{\mathbb{Z}} \mathbb{Z}_{q}}{N \otimes_{\mathbb{Z}} \mathbb{Z}_{q}}}_{K_{q}(S)} \rightarrow \underbrace{\frac{L \otimes_{\mathbb{Z}} \mathbb{Z}_{q}}{M \otimes_{\mathbb{Z}} \mathbb{Z}_{q}}}_{(L / M) \otimes_{\mathbb{Z}} \mathbb{Z}_{q}} \rightarrow 0
$$

Here we have used on the two ends of the sequence the fact ${ }^{4}$ that for any pair of nested abelian groups $B \subset A$, one has

$$
\left(A \otimes_{\mathbb{Z}} \mathbb{Z}_{q}\right) /\left(B \otimes_{\mathbb{Z}} \mathbb{Z}_{q}\right) \cong(A / B) \otimes_{\mathbb{Z}} \mathbb{Z}_{q}
$$

Furthermore, it is easy to see that both $M / N$ and $L / M$ are isomorphic to $\mathbb{Z}_{2}$ :

- The isomorphism $M / N \cong \mathbb{Z}_{2}$ comes from choosing any $\mathbb{Z}$-basis for the lattice $M$, thus identifying $M \cong \mathbb{Z}^{|V|}$, and noting that under this identification, $N$ is identified with the index 2 sublattice $\left\{x \in \mathbb{Z}^{|V|}: \sum_{v \in V} x_{v} \in 2 \mathbb{Z}\right\}$.

- The isomorphism $L / M \cong \mathbb{Z}_{2}$ is equivalent to the assertion that the square (unsigned) edge-node incidence matrix having columns indexed by the nodes $V$ and rows indexed by the edges $S$ will have determinant \pm 2 . This is a well-known fact for connected unicyclic graphs $S$ whose unique cycle $C$ is odd; see, e.g., [18, p. 560, proof of Thm. 3.3]. It is easily proven by first checking that the determinant is scaled by \pm 1 when one removes a row and column corresponding to a leaf edge and its incident leaf vertex in $S$. This reduces the assertion to the case where $S=C$ is just an odd cycle itself, where the determinant can be calculated directly via Laplace expansion.

Hence both of the outer terms $(M / N) \otimes_{\mathbb{Z}} \mathbb{Z}_{q},(L / M) \otimes_{\mathbb{Z}} \mathbb{Z}_{q}$ in the short exact sequence (19) are isomorphic to $\mathbb{Z}_{2} \otimes_{\mathbb{Z}} \mathbb{Z}_{q}$, which vanishes for $p$ odd and equals $\mathbb{Z}_{2}$ for $p=2$. Thus (19) shows that $K_{q}(S)$ vanishes for $p$ odd, and shows for $p=2$ that $K_{q}(S)$ is either $\mathbb{Z}_{2}^{2}$ or $\mathbb{Z}_{4}$. To distinguish these possibilities when $p=2$, we analyze the additive orders of each edge $e$ in $S$ as elements of $K_{q}(S)$.

Note that for any leaf edge $e$ in $S$, say with leaf vertex $v$, one has $e=\delta_{S}(v)$, and hence $2 e=\delta_{S}(v) \equiv 0$ in $K_{q}(S)$. Thus using a leaf-induction, one sees that any edge $e$ in $S \backslash C$ has $2 e \equiv 0$ in $K_{q}(S)$.

\footnotetext{
${ }^{4}$ That is, taking tensor products $(-)_{\otimes \mathbb{Z}} \mathbb{Z}_{q}$ is right exact, so when applied to the exact sequence

$$
B \rightarrow A \rightarrow A / B \rightarrow 0
$$
}

it gives the exact sequence

$$
B \otimes_{\mathbb{Z}} \mathbb{Z}_{q} \rightarrow A \otimes_{\mathbb{Z}} \mathbb{Z}_{q} \rightarrow(A / B) \otimes_{\mathbb{Z}} \mathbb{Z}_{q} \rightarrow 0 .
$$



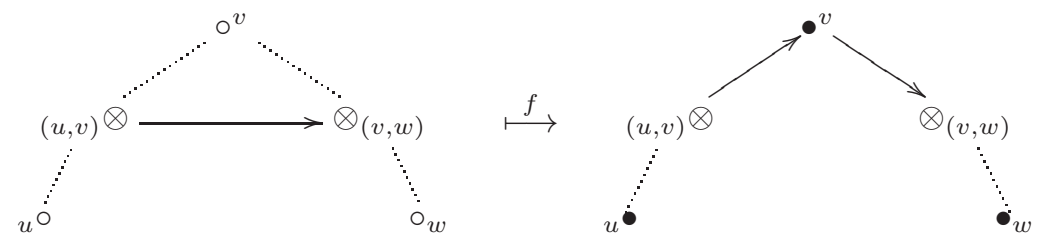

Figure 4 . The action of $f$ on a single edge of line $G$.

Meanwhile, we claim that for any edge $e=\{v, w\}$ in $C$, one has $c \cdot e \equiv 0$ in $K_{q}(S)$ if and only if $|V| \cdot c$ lies in $4 \mathbb{Z}_{q}$. To see the claim, assume that $c \cdot e=0$ in $K_{q}(S)$ for some $c$ in $\mathbb{Z}_{q}$. This means one has a sum $\sum_{v \in V} c_{v} \delta_{G}(v)=c \cdot e$ with $\sum_{v \in V} c_{v} \in 2 \mathbb{Z}_{q}$. This happens if and only if the sum has zero coefficient on all edges $e^{\prime}$ in $S \backslash\{e\}$. Applying this for the edges $e^{\prime}$ in $C \backslash\{e\}$, one concludes that $c_{v}=c_{w}$, and hence $c=c_{v}+c_{w}=2 c_{v}$. Applying this for the remaining edges $e^{\prime}$ in $S \backslash C$, one concludes that $c_{w}= \pm c_{v}$ for all $w$ in $V$. But then the condition that $\sum_{w \in V} c_{w}$ lies in $2 \mathbb{Z}_{q}$ means that $|V| \cdot c_{v}$ also lies in $2 \mathbb{Z}_{q}$, i.e., that $|V| \cdot c=2|V| \cdot c_{v}$ lies in $4 \mathbb{Z}_{q}$. One concludes that edges $e$ in $C$ have order 2 when $|V|$ is even, and order 4 when $|V|$ is odd. Since every edge $e$ in $S \backslash C$ has $2 e \equiv 0$ in $K_{q}(S)$, this implies $K_{q}(S) \cong \mathbb{Z}_{2}^{2}$ when $|V|$ is even and $K_{q}(S) \cong \mathbb{Z}_{4}$ when $|V|$ is odd.

\section{Proof of Theorem 1.3}

Recall here the statement of Theorem 1.3.

Theorem 1.3. For any connected d-regular simple graph $G$ with $d \geq 3$, there is a group homomorphism

$$
K(\text { line } G) \stackrel{f}{\rightarrow} K(\operatorname{sd} G)
$$

whose kernel-cokernel exact sequence takes the form

$$
0 \rightarrow \mathbb{Z}_{d}^{\beta(G)-2} \oplus C \rightarrow K(\text { line } G) \stackrel{f}{\rightarrow} K(\operatorname{sd} G) \rightarrow C \rightarrow 0
$$

in which the cokernel $C$ is the following cyclic d-torsion group:

$$
C= \begin{cases}0 & \text { if } G \text { is non-bipartite and } d \text { is odd }, \\ \mathbb{Z}_{2} & \text { if } G \text { is non-bipartite and } d \text { is even, } \\ \mathbb{Z}_{d} & \text { if } G \text { is bipartite. }\end{cases}
$$

6.1. Defining the morphism $f$. We begin our proof of the theorem by first defining a linear map $f: \mathbb{R}^{E_{\text {line } G}} \rightarrow \mathbb{R}^{E_{\mathrm{sd} G}}$ which will turn out to be a morphism of rational orthogonal decompostions.

Definition 6.1. Define a $\mathbb{R}$-linear map $f: \mathbb{R}^{E_{\text {line } G} \rightarrow \mathbb{R}^{E_{\text {sd } G}} \text { by setting }}$

$$
f(u v, v w)=(u v, v)+(v, v w)
$$

for every pair of edges $\{u, v\},\{v, w\}$ of $G$ incident at some vertex $v$ (see Figure 4 ). Equivalently, the adjoint map $f^{t}$ is defined by

$$
f^{t}(u v, v)=\sum_{w \in V:\{v, w\} \in E}(u v, v w) .
$$

The following definitions and lemma will be useful both for showing that $f$ gives a morphism, and in our later analysis. 
Definition 6.2. Given a directed cycle

$$
C=\left\{\left(v_{1}, v_{2}\right),\left(v_{2}, v_{3}\right), \ldots,\left(v_{m-1}, v_{m}\right),\left(v_{m}, v_{1}\right)\right\}
$$

in $G$, let

$$
\begin{aligned}
\operatorname{sd} C & :=\left\{\left(v_{1}, v_{1} v_{2}\right),\left(v_{1} v_{2}, v_{2}\right),\left(v_{2}, v_{2} v_{3}\right),\left(v_{2} v_{3}, v_{3}\right), \ldots\right\} \\
\text { line } C & :=\left\{\left(v_{1} v_{2}, v_{2} v_{3}\right),\left(v_{2} v_{3}, v_{3} v_{4}\right), \ldots,\left(v_{m-1} v_{m}, v_{m} v_{1}\right),\left(v_{m} v_{1}, v_{1} v_{2}\right)\right\}
\end{aligned}
$$

denote corresponding cycles in $\operatorname{sd} G$, line $G$.

Cycles in line $G$ of the form line $C$ where $C$ is a cycle of $G$ will be called global cycles. A cycle in line $G$ will be called local (to vertex $v$ ) if every vertex $v_{i} v_{j}$ of line $G$ visited by the cycle has $v \in\left\{v_{i}, v_{j}\right\}$.

Lemma 6.3. Let $G$ be a graph, and let $\{C\}$ be a set of directed cycles indexing a spanning set $\{z(C)\}$ for the cycle space $Z_{G}$. Then

(1) $Z_{\mathrm{sd} G}$ will be spanned by the incidence vectors $\{z(\operatorname{sd} C)\}$ of the associated subdivided cycles, and

(2) $Z_{\text {line } G}$ will be spanned by the incidence vectors $\{z$ (line $\left.C)\right\}$ for their associated global cycles together with all local cycles.

Proof. Assertion (1) of was implicitly used in Section 3.3, and should be clear either from elementary algebraic topology or from the discussion of bases for $Z_{G}$ coming from spanning forests at the beginning of Section 3.1.

For assertion (2), given any directed cycle in line $G$, put an equivalence relation on its edges by taking the transitive closure of the following relation: two consecutive edges $(u v, v w),(v w, w x)$ in the cycle are equivalent if there exists a vertex $y$ of $G$ contained in $\{u, v\} \cap\{v, w\} \cap\{w, x\}$. The global cycles in line $G$ are by definition those in which the equivalence classes for this relation all have cardinality two (N.B.: here one is using the assumption that $G$ is simple). Given a cycle $z$ in line $G$ that contains equivalence classes of size at least 3 , it is easy to see that one can always add a local cycle to $z$ and reduce the number of such equivalence classes: if the equivalence class and its neighbors in $z$ correspond to these terms

$$
\cdots+\left(a b_{1}, y b_{1}\right)+\left(y b_{1}, y b_{2}\right)+\left(y b_{2}, y b_{3}\right)+\cdots+\left(y b_{t-1}, y b_{t}\right)+\left(y b_{t}, b_{t} c\right)+\cdots
$$

where $a, c \neq y$, then subtracting the local cycle

$$
\left(y b_{1}, y b_{2}\right)+\left(y b_{2}, y b_{3}\right)+\cdots+\left(y b_{t-1}, y b_{t}\right)+\left(y b_{t}, y b_{1}\right)
$$

gives a result that looks locally like

$$
\cdots+\left(a b_{1}, y b_{1}\right)+\left(y b_{1}, y b_{t}\right)+\left(y b_{t}, b_{t} c\right)+\cdots .
$$

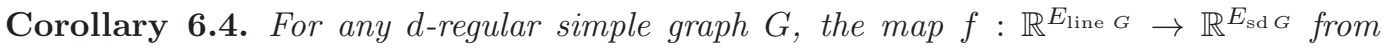
Definition 6.1 is a morphism of the associated rational orthogonal decompositions, and hence induces a group homomorphism

$$
f: K(\text { line } G) \rightarrow K(\operatorname{sd} G) .
$$

Proof. By Proposition 2.3, one must show both $f\left(Z_{\text {line } G}\right) \subset Z_{\text {sd } G}$ and $f^{t}\left(Z_{\text {sd } G}\right) \subset Z_{\text {line } G}$.

To show $f\left(Z_{\text {line } G}\right) \subset Z_{\text {sd } G}$, using Lemma 6.3(ii), it suffices to show that $f$ takes both global and local cycles in line $G$ to cycles in $Z_{\text {sd } G}$. This is easy (and requires no assumption about the $d$-regularity of $G$ ): local cycles map to 0 under $f$, and a global cycle of the form line $C$ satisfies $f(z($ line $C))=z(\operatorname{sd} C)$. 

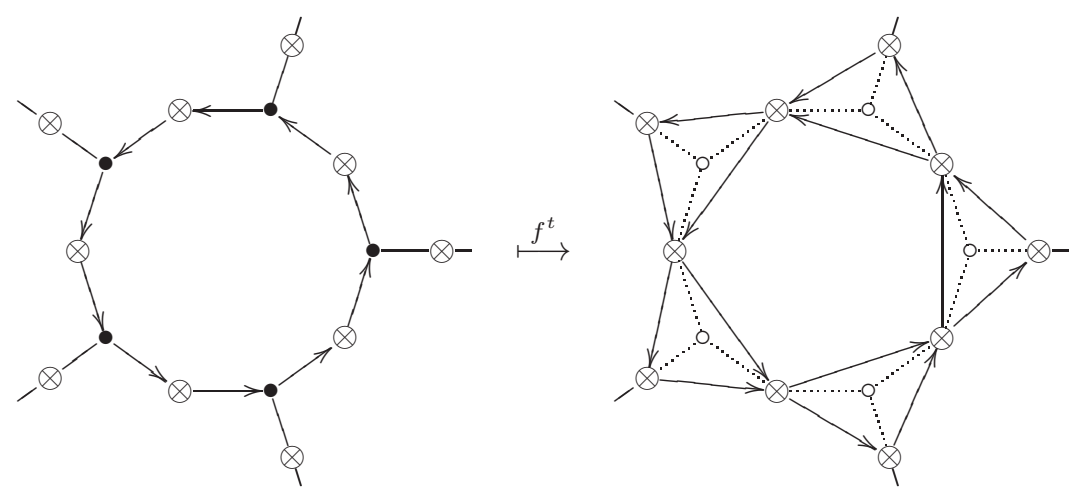

Figure 5. An example of a subdivided cycle in sd $G$, and its image under $f^{t}$ in line $G$ when $G$ is 3-regular.

To show $f^{t}\left(Z_{\text {sd } G}\right) \subset Z_{\text {line } G}$, using Lemma 6.3(i), it suffices to show for every directed cycle $C$ in $G$ that $f$ takes the subdivided cycle

$$
z(\operatorname{sd} C)=\left(v_{1} v_{2}, v_{2}\right)+\left(v_{2}, v_{2} v_{3}\right)+\left(v_{2} v_{3}, v_{3}\right)+\left(v_{3}, v_{3} v_{4}\right)+\cdots+\left(v_{k} v_{1}, v_{1}\right)+\left(v_{1}, v_{1} v_{2}\right)
$$

to a sum of cycles in $Z_{\text {line } G}$. The regularity of $G$ implies that each $v_{i}$ has $d-2$ neighbors off the cycle; label them $u_{i}^{1}, \ldots, u_{i}^{d-2}$. Then one can write

$$
\left(f^{t}\right)(z(\operatorname{sd} C))=2 z(\text { line } C)+\zeta_{1}+\cdots+\zeta_{d-2}
$$

where for $j=1,2, \ldots, d-2$ one defines the element of $Z_{\text {line } G}$

$$
\begin{aligned}
\zeta_{j}:=\left(v_{1} v_{2}, v_{2} u_{2}^{j}\right)+\left(v_{2} u_{2}^{j}, v_{2} v_{3}\right)+\left(v_{2} v_{3}, v_{3} u_{3}^{j}\right) & +\left(v_{3} u_{3}^{j}, v_{3} v_{4}\right)+\cdots \\
& +\left(v_{k} v_{1}, v_{1} u_{1}^{j}\right)+\left(v_{1} u_{1}^{j}, v_{1} v_{2}\right) .
\end{aligned}
$$

An example with $d=3$ is shown in Figure 5, depicting the subdivided cycle $\operatorname{sd} C$ in $\operatorname{sd} G$, and then its image under $f^{t}$ in line $G$, which decomposes into 2 copies of the inner cycle line $C$ along with $1(=d-2)$ outer cycle $\zeta_{1}$.

\subsection{The kernel and cokernel of $f$ are $d$-torsion.}

Proposition 6.5. For any d-regular connected graph $G$, both maps

$$
\begin{aligned}
& f^{t} f: K(\text { line } G) \rightarrow K(\text { line } G) \\
& f f^{t}: K(\operatorname{sd} G) \rightarrow K(\operatorname{sd} G)
\end{aligned}
$$

are scalar multiplications by $d$. 
Proof. The proofs of these are straightforward computations:

$$
\begin{aligned}
f^{t} f(u v, v w) & =f^{t}(u v, v)+f^{t}(v, v w) \\
& =\sum_{x \in V:\{v, x\} \in E}(u v, v x)+(x v, v w) \\
& =d \cdot(u v, v w)+\sum_{x \in V:\{v, x\} \in E}((u v, v x)+(x v, v w)+(v w, u v)) \\
& =d \cdot(u v, v w) \bmod Z_{\text {line } G .} \\
f f^{t}(u v, v) & =\sum_{x \in V:\{v, x\} \in E} f(u v, v x) \\
& =\sum_{x \in V:\{v, x\} \in E}(u v, v)+(v, v x) \\
& =d \cdot(u v, v)+\sum_{x \in V:\{v, x\} \in E}(v, v x) \\
& =d \cdot(u v, v) \bmod B_{\mathrm{sd} G} .
\end{aligned}
$$

Corollary 6.6. For any d-regular connected graph $G$, both $\operatorname{ker}(f)$ and $\operatorname{coker}(f)$ are all d-torsion.

Proof. For $x \in \operatorname{ker}(f)$ and $y \in \operatorname{coker}(f)$, one has

$$
\begin{aligned}
& d \cdot x=f^{t} f(x)=f^{t}(0)=0, \\
& d \cdot y=f f^{t}(y) \in \operatorname{im}(f) .
\end{aligned}
$$

\subsection{Analyzing the cokernel.}

Proposition 6.7. For any d-regular connected graph $G$, the group $C:=\operatorname{coker}(f)$ is a cyclic group as described in Theorem 1.3.

Proof. We will use the presentation

$$
C:=\operatorname{coker}(f):=K(\operatorname{sd} G) / \operatorname{im}(f)=\mathbb{Z}^{E_{\text {sd } G}} /\left(B_{\mathrm{sd} G}+Z_{\mathrm{sd} G}+\operatorname{im}(f)\right),
$$

which follows from our first definition of the critical group (as in Section 2).

To see that $C$ is cyclic, note that there are two ways for a pair of edges in $\operatorname{sd} G$ to be incident at a vertex, and in either case their images in $C$ will differ by a sign:

$$
\begin{aligned}
& (u, u v)=-(u v, v) \quad \bmod B_{\mathrm{sd} G}, \\
& (u v, v)=-(v, v w) \quad \bmod \operatorname{im}(f) .
\end{aligned}
$$

Since $G$ is connected, this shows $C$ is cyclic, generated by the image of any directed edge of sd $G$. Furthermore, it is a quotient of $\mathbb{Z}_{d}$ by Corollary 6.6.

When $G$ is bipartite, in order to show $C=\mathbb{Z}_{d}$, it will suffice to exhibit a surjection $C \rightarrow \mathbb{Z}_{d}$. Let the vertex set $V$ for $G$ have bipartition $V=V_{1} \sqcup V_{2}$ and assume that all the edges of $G$ are oriented from $V_{1}$ to $V_{2}$. Define an abelian group homomorphism

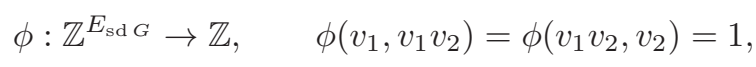

where $v_{i} \in V_{i}$ for $=1,2$. One can check that each of the three subgroups $B_{\mathrm{sd} G}, Z_{\mathrm{sd} G}, \operatorname{im}(f)$ by which one mods out in (20) is mapped via $\phi$ into the subgroup $d \mathbb{Z}$ :

- Any directed cycle $C$ in sd $G$ has $\phi(z(C))=0$ (due to the fact that $C$ will have even length), 
- any edge $e$ of line $G$ has $\phi(f(e))=0$,

- any vertex $v_{1} v_{2}$ in $\operatorname{sd} G$ has $\phi\left(b_{\mathrm{sd}} G\left(v_{1} v_{2}\right)\right)=0$,

- any vertex $v_{i}$ in $\operatorname{sd} G$ has $\phi\left(b_{\text {sd } G}\left(v_{i}\right)\right)=(-1)^{i-1} d$, where $i=1,2$.

Thus $\phi$ induces a surjection from $C$ onto $\mathbb{Z}_{d}$, as desired.

If $G$ is not bipartite, it contains some (directed) odd cycle $C$. Pick any directed edge $e$ in the subdivision sd $C$ and use the two relations (a), (b) to rewrite it successively as \pm the other directed edges in the cycle. It changes sign each time one uses (a) to pass through a vertex of sd $C$ that comes from an edge of $G$. Since there are an odd number of such edges in the cycle, it will change sign an odd number of times before it returns, yielding

$$
e=-e \bmod B_{\operatorname{sd} G}+\operatorname{im}(f) .
$$

Hence $2 e=0$ in $C$, so $C$ is a quotient of $\mathbb{Z}_{2}$.

Since $C$ is also a quotient of $\mathbb{Z}_{d}$, when $d$ is odd, one must have $C=0$. When $d$ is even, consider the index 2 sublattice $\Lambda$ of $\mathbb{Z}^{E_{\mathrm{sd} G}}$ consisting of those vectors whose sum of coordinates is even. Without any parity assumption on $d$, it is true that $\operatorname{im}(f) \subset \Lambda$ (by definition of $f$ ) and $Z_{\operatorname{sd} G} \subset \Lambda$ (because the subdivided cycles sd $C$ have evenly many edges). The assumption that $d$ is even implies that $B_{\mathrm{sd} G}$ also lies in $\Lambda: B_{\mathrm{sd} G}$ is generated by the bonds in $\operatorname{sd} G$ of the form $b_{\operatorname{sd} G}(v)$ for vertices $v$ of $\operatorname{sd} G$, and every vertex in $\operatorname{sd} G$ has degree either 2 or $d$. Consequently, the presentation (20) shows that $C$ surjects onto

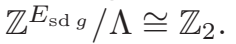

6.4. Analyzing the kernel. It remains to understand $\operatorname{ker}(f)$, or equivalently by Proposition 2.6, to understand its Pontrjagin dual

$$
\operatorname{coker}\left(f^{t}\right)=\mathbb{Z}^{E_{\text {line } G}} /\left(Z_{\text {line } G}+B_{\text {line } G}+\operatorname{im}\left(f^{t}\right)\right) .
$$

This will come about by reformulating this presentation, in order to analyze it locally.

Definition 6.8. For each vertex $v \in V_{G}$ of a $d$-regular simple graph $G=\left(V_{G}, E_{G}\right)$, define inside line $G$ the $d$-clique local to $v$

$$
K_{d}^{(v)}=\left(V\left(K_{d}^{(v)}\right), E\left(K_{d}^{(v)}\right)\right)
$$

to be the vertex-induced subgraph of line $G$ on the vertex set

$$
V\left(K_{d}^{(v)}\right):=\left\{v w: v w \in E_{G}\right\} .
$$

Note that the edges of line $G$ form a disjoint decomposition

$$
E_{\text {line } G}=\bigsqcup_{v \in V_{G}} E\left(K_{d}^{(v)}\right)
$$

since $G$ was assumed to be a simple graph. Also note that a cycle in line $G$ is local to vertex $v$, as in Definition 6.2, if and only if it is supported on the edges $E\left(K_{d}^{(v)}\right)$. If one lets $Z_{\text {line } G}^{\text {global }}$ be the span of global cycles $\left\{z_{\text {line }} C\right\}$ coming from any spanning set of cycles $\left\{z_{C}\right\}$ for $Z_{G}$, then Lemma 6.3 (ii) implies

$$
Z_{\text {line } G}=Z_{\text {line } G}^{\text {local }}+Z_{\text {line } G}^{\text {global }} \text {. }
$$

To simplify the presentation (21), note that for a vertex $v w$ of line $G$, the bond

$$
b_{\text {line } G}(v w)=f^{t}(v w, v)+f^{t}(v w, w)
$$


lies in $\operatorname{im}\left(f^{t}\right)$, and consequently, $B_{\text {line } G} \subset \operatorname{im}\left(f^{t}\right)$. Note also that the decomposition (22) leads to a family of compatible direct sum decompositions

$$
\begin{aligned}
\mathbb{Z}^{E_{\text {line } G}} & =\bigoplus_{v \in V_{G}} \mathbb{Z}^{E\left(K_{d}^{(v)}\right)} \\
Z_{\text {line } G}^{\text {local }} & =\bigoplus_{v \in V_{G}} Z_{K_{d}^{(v)}} \\
\operatorname{im}\left(f^{t}\right) & =\bigoplus_{v \in V_{G}} B_{K_{d}^{(v)}}
\end{aligned}
$$

This gives the simplified presentation

$$
\begin{aligned}
\operatorname{coker}\left(f^{t}\right) & =\left(\bigoplus_{v \in V_{G}} \mathbb{Z}^{E\left(K_{d}^{(v)}\right)} /\left(B_{K_{d}^{(v)}}+Z_{K_{d}^{(v)}}\right)\right) / Z_{\text {line } G}^{\text {global }} \\
& =\left(\bigoplus_{v \in V_{G}} K\left(K_{d}^{(v)}\right)\right) / Z_{\text {line } G}^{\text {global }}
\end{aligned}
$$

We use this presentation to prove the following lemma, which together with Proposition 6.7 immediately implies Theorem 1.3.

Lemma 6.9. For a connected d-regular graph $G$,

$$
\operatorname{ker}(f) \cong \mathbb{Z}_{d}^{\beta(G)-2} \oplus C
$$

where $C:=\operatorname{coker}(f)$ is as described in Theorem 1.3.

Proof. We claim that it suffices to prove these two bounds on $\operatorname{ker}(f)$ :

(i) There is a surjection $\operatorname{ker}(f) \rightarrow \mathbb{Z}_{d}^{\beta(G)-2}$ and,

(ii) $\operatorname{ker}(f)$ can be generated by $\beta(G)-1$ elements.

To see this claim, note that since $\operatorname{ker}(f)$ is all $d$-torsion by Corollary 6.6 , assertion (ii) would imply a surjection $\mathbb{Z}_{d}^{\beta(G)-1} \rightarrow \operatorname{ker}(f)$. Together with (i), this would imply $\operatorname{ker}(f) \cong$ $\mathbb{Z}_{d}^{\beta(G)-2} \oplus C^{\prime}$ for some cyclic group $C^{\prime}$. But then exactness of the sequence

$$
0 \rightarrow \underbrace{\operatorname{ker}(f)}_{\mathbb{Z}_{d}^{\beta(G)-2} \oplus C^{\prime}} \rightarrow K(\text { line } G) \stackrel{f}{\rightarrow} K(\operatorname{sd} G) \rightarrow \underbrace{\operatorname{coker}(f)}_{C} \rightarrow 0
$$

forces

$$
\left(d^{\beta(G)-2}\left|C^{\prime}\right|\right)|K(\operatorname{sd} G)|=\mid K(\text { line } G)|| C \mid .
$$

From this equation and equation (2) one deduces $\left|C^{\prime}\right|=|C|$. Since both $C^{\prime}$ and $C$ are cyclic, this means $C^{\prime} \cong C$, as desired.

In the proofs of assertions (i) and (ii), one uses the fact that $\operatorname{ker}(f)=\operatorname{coker}\left(f^{t}\right)$. Moreover, setting $n:=\left|V_{G}\right|$, one can rewrite the direct sum from (23) as

$$
\bigoplus_{v \in V_{G}} K\left(K_{d}^{(v)}\right) \cong \bigoplus_{v \in V_{G}} \mathbb{Z}_{d}^{d-2} \cong \mathbb{Z}_{d}^{n(d-2)}
$$

For assertion (i), we use some easy numerology. Note that $Z_{\text {line } G}^{\text {global }}$ can be generated by $\beta(G)$ elements, and also that

$$
\beta(G)=\left|E_{G}\right|-\left|V_{G}\right|+1=\frac{d n}{2}-n+1=\frac{n(d-2)}{2}+1
$$


so that

$$
n(d-2)-\beta(G)=\beta(G)-2 .
$$

Since it is easily seen that that any quotient of an abelian group $\mathbb{Z}_{d}^{a}$ by a subgroup that can be generated by $b$ elements will have a surjection to $\mathbb{Z}_{d}^{a-b}$, one can apply this with $a=n(d-2)$ and $b=\beta(G)$ to the presentation (23), and conclude that there is a surjection $\operatorname{coker}\left(f^{t}\right) \rightarrow \mathbb{Z}_{d}^{\beta(G)-2}$.

For assertion (ii), the idea will be to start with the

$$
n(d-2)=2(\beta(G)-1)
$$

generators in (24), and use (all but one of) the $\beta(G)$ generating global cycles in $Z_{\text {line } G}^{\text {global }}$ to rewrite them in terms of other generators, with $\beta(G)-1$ generators left. This will be achieved by removing the vertices from $G$ one at a time in a certain order, in order to control the rewriting process.

To this end, order the vertices $V_{G}$ as $v_{1}, v_{2}, \ldots, v_{n}$ in such a way that the vertex-induced subgraphs

$$
\begin{aligned}
G_{i} & :=G \backslash\left\{v_{1}, v_{2}, \ldots, v_{i-1}\right\} \\
& \left(\text { so } G_{1}:=G, \text { and } G_{n} \text { has one vertex } v_{n}\right)
\end{aligned}
$$

satisfy

$$
d_{i}:=\operatorname{deg}_{G_{i}}\left(v_{i}\right)<d \text { for } i \geq 2 .
$$

For each $i \geq 1$, partition the $d_{i}$ neighbors $v_{i}$ in $G_{i}$ into blocks $A_{1}, A_{2}, \ldots, A_{c_{i}}$ according to the connected components of $G_{i+1}$ in which they lie. The number $c_{i}$ of such components coincides with the number of connected components in $G_{i+1}$ into which the connected component of $v_{i}$ in $G_{i}$ splits after removing $v_{i}$. Define

$$
\Delta_{i}:=d_{i}-c_{i}=\beta\left(G_{i}\right)-\beta\left(G_{i+1}\right),
$$

where the last equality follows from the Euler relation for graphs:

$$
\left|V_{G}\right|-\left|E_{G}\right|=\mid\{\text { connected components of } G\}|-| \beta(G) \mid \text {. }
$$

Consequently,

$$
\Delta_{1}+\Delta_{2}+\cdots+\Delta_{n-1}=\beta\left(G_{1}\right)-\beta\left(G_{n}\right)=\beta(G) .
$$

Our goal will then be to find $\Delta_{i}$ minimal generators of (24) to remove at each stage $i \geq 2$ (and at the first stage $i=1$, remove one fewer, that is, $\Delta_{1}-1=d-2$ of them). This would leave a generating set for $\operatorname{coker}\left(f^{t}\right)$ of cardinality $n(d-2)-(\beta(G)-1)=\beta(G)-1$, as desired.

For $i \geq 2$, inside the clique $K_{d}^{\left(v_{i}\right)}$ local to $v_{i}$, choose a forest $F_{i}$ of edges having $c_{i}$ components which are spanning trees on each of the subsets $\left\{v_{i} x: x \in A_{j}\right\}$ for $j=1,2, \ldots, c_{i}$. Note that

$$
\left|F_{i}\right|=\sum_{j=1}^{c_{i}}\left(\left|A_{j}\right|-1\right)=d_{i}-c_{i}=\Delta_{i} .
$$

Also note that the forest $F_{i}$ manages to avoid touching at least one vertex in the $d$-clique $K_{d}^{\left(v_{i}\right)}$, namely any vertex of the form $v_{i} v_{k}$ in which $\left\{v_{i} v_{k}\right\} \in E_{G}$ and $k<i$; there will exist at least one such $k$ since by construction, $\operatorname{deg}_{G_{i}}\left(v_{i}\right)=d_{i}<d=\operatorname{deg}_{G}\left(v_{i}\right)$.

Hence by Proposition 3.3, the edges in $F_{i}$ give $\Delta_{i}$ generators that could be completed to a set of $d-2$ minimal generators for $K\left(K_{d}^{\left(v_{i}\right)}\right) \cong \mathbb{Z}_{d}^{d-2}$. Each of these generators in $F_{i}$ can be re-written, using a cycle in $Z_{\text {line } G}^{\text {global }}$, in terms of generators from $K\left(K^{\left(v_{k}\right)}\right)^{\text {'s }}$ that have $k>i$, as follows. Given any edge $\left(v_{i} x, v_{i} x^{\prime}\right)$ in $F_{i}$, there is a path from $x$ to $x^{\prime}$ in $G_{i+1}$ (because $x, x^{\prime}$ lie in the same component of $G_{i+1}$ by construction), and hence a directed 


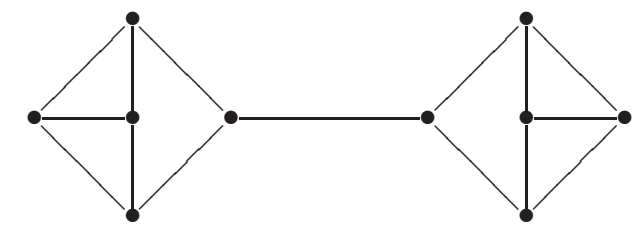

FiguRE 6. A 3-regular connected graph which is not 2-edge-connected.

cycle $C$ in $G_{i}$ going from $v_{i}$ to $x$ then through this path to $x^{\prime}$ and back to $v_{i}$. The global cycle $z$ (line $C$ ) allows one to rewrite $\left(v_{i} x, v_{i} x^{\prime}\right)$ as desired.

The only difference for $i=1$ is that, even when $\Delta_{1}=d-1$ (that is, when $c_{1}=1$ ), in this situation choose $F_{i}$ to have at most $d-2$ edges (that is, remove any edge from the forest $F_{1}$ if $c_{1}=1$ ). This modification ensures that one can still apply Proposition 3.3 and rewrite all of the generators of $K\left(K_{d}^{\left(v_{1}\right)}\right)$ corresponding to the edges of $F_{1}$.

Remark 6.10. One should remark that for a connected, $d$-regular graph $G$, the extra hypothesis in Theorem 1.3 that $G$ is 2-edge-connected is well-known (see, e.g., [9]) to be superfluous when $d$ is even: a connected graph $G$ with all vertices of even degree cannot have a cut-edge, as the two components created by the removal of this edge would each be graphs having exactly one vertex of odd degree, an impossibility.

However, when $d$ is odd, the extra hypothesis of 2-edge-connectivity need not follow. For example, the 3-regular graph shown in Figure 6 is connected, but not 2-edge-connected.

\section{Proof of Corollary 1.4}

In this section we prove Corollary 1.4. Informally, the corollary states that critical group of $G$ determines the critical group of line $G$ in a simple way.

Corollary 1.4. For $G$ a simple, connected, $d$-regular graph with $d \geq 3$ which is nonbipartite, after expressing uniquely

$$
K(G) \cong \bigoplus_{i=1}^{\beta(G)} \mathbb{Z}_{d_{i}}
$$

with $d_{i}$ dividing $d_{i+1}$, one has

$$
K(\text { line } G) \cong\left(\begin{array}{ll}
\beta(G)-2 \\
\bigoplus_{i=1} \mathbb{Z}_{2 d d_{i}}
\end{array}\right) \oplus \begin{cases}\mathbb{Z}_{2 d_{\beta(G)-1}} \oplus \mathbb{Z}_{2 d_{\beta(G)}} & \text { for }|V| \text { even } \\
\mathbb{Z}_{4 d_{\beta(G)-1}} \oplus \mathbb{Z}_{d_{\beta(G)}} & \text { for }|V| \text { odd. }\end{cases}
$$

Proof. Let $K:=K$ (line $G$ ), and fix a prime $p$. Our goal is to show that the $p$-primary component of $K$ matches that of the group on the right side of (25).

The hypotheses of the theorem allow one to apply the nonbipartite cases of Theorem 1.2 and Theorem 1.3. The former asserts that

$$
K / p^{k(p)} K \cong \mathbb{Z}_{p^{k(p)}}^{\beta(G)-2} \oplus \begin{cases}0 & \text { for } p \text { odd } \\ \mathbb{Z}_{2}^{2} & \text { for } p=2,|V| \text { even } \\ \mathbb{Z}_{4} & \text { for } p=2,|V| \text { odd }\end{cases}
$$

while the latter gives an exact sequence

$$
0 \rightarrow \mathbb{Z}_{d}^{\beta(G)-2} \oplus \mathbb{Z}_{\operatorname{gcd}(2, d)} \rightarrow K \rightarrow K(\operatorname{sd} G) \rightarrow \mathbb{Z}_{\operatorname{gcd}(2, d)} \rightarrow 0 .
$$


In analyzing the $p$-primary component $\operatorname{Syl}_{p}(K)$, it is convenient to define the type of a finite abelian $p$-group $A$ as the unique integer partition $\nu=\left(\nu_{1} \geq \nu_{2} \geq \cdots\right)$ for which $A \cong \bigoplus_{i \geq 1} \mathbb{Z}_{p^{\nu_{i}}}$. Let $\mu, \lambda$ denote the types of $\operatorname{Syl}_{p}(K(G))$, $\operatorname{Syl}_{p}(K)$, where we think of both $\mu, \lambda$ as partitions with $\beta(G)$ parts (allowing some parts to be 0 ). Note that Proposition 3.2 asserts, in this language, that $\operatorname{Syl}_{p}(K(\operatorname{sd} G))$ has type $\mu$ for $p$ odd and type $\mu+\left(1^{\beta(G)}\right)$ for $p=2$.

A basic fact from the theory of Hall polynomials [14, Chapter II Section 9] says that there exist short exact sequences of abelian $p$-groups

$$
0 \rightarrow A \rightarrow B \rightarrow C \rightarrow 0
$$

in which $A, B, C$ have types $\nu, \lambda, \mu$, respectively, if and only if the Littlewood-Richardson (or $L R$ ) coefficient $c_{\mu, \nu}^{\lambda}$ does not vanish. The combinatorial rephrasing of this $L R$-condition is as follows: There must exist at least one column-strict tableau (which we will call an $L R$ tableau) of the skew-shape $\lambda / \mu$ having content $\nu$, for which the word obtained by reading the tableau (in English notation) from right-to-left in each row, starting with the top row, is Yamanouchi. Here the Yamanouchi condition means that within each initial segment of the word, and for each value $i \geq 1$, the number of occurrences of $i+1$ is at most the number of occurrences of $i$. See [14, Chapter I §9] and [17, Appendix §A1.3] for more on these notions.

Suppose that $p$ is odd. Then $k(p)$ is the largest power such that $p^{k(p)}$ divides $d$, so taking the $p$-primary components in (27), we obtain the following short exact sequence:

$$
0 \longrightarrow \underbrace{\mathbb{Z}_{p^{k(p)}}^{\beta(G)-2}}_{\text {type } \nu=\left(k(p)^{\beta(G)-2}\right)} \longrightarrow \underbrace{\operatorname{Syl}_{p} K}_{\text {type } \lambda} \longrightarrow \underbrace{\operatorname{Syl}_{p}(K(\operatorname{sd} G))}_{\text {type } \mu} \longrightarrow 0
$$

where $\lambda$ has at most $\beta(G)-2$ nonzero parts by (26). Since nonvanishing of the LR-coefficient $c_{\mu, \nu}^{\lambda}$ forces $\mu \subset \lambda$, it must be that $\mu$ also has at most $\beta(G)-2$ nonzero parts. Furthermore, one can check that column-strictness together with the Yamanouchi condition on the reading word of an LR-tableau of shape $\lambda / \mu$ and content $\nu=\left(k(p)^{\beta(G)-2}\right)$ uniquely determine the tableau: It must have each entry in row $i$ equal to $i$ for $i=1,2, \ldots, \beta(G)-2$. This forces $\lambda_{i}=\mu_{i}+k(p)$ for $i=1,2, \ldots, \beta(G)-2$, and hence $\lambda$ agrees with the type of the $p$-primary component on the right side of $(25)$.

Suppose that $p=2$, so that $2^{k(p)-1}$ divides $d$, but $2^{k(p)}$ does not.

When $d$ is odd we have that $k(p)=1$. On the other hand, taking the 2-primary components in (27) shows that $\operatorname{Syl}_{2} K \cong \operatorname{Syl}_{2}(K(\operatorname{sd} G))$, so $\lambda=\mu+\left(1^{\beta(G)}\right)$. Since $d$ is odd, $|V|$ must be even (as the $d$-regularity of $G$ forces $d|V|=2|E|$ ), so this $\lambda$ again agrees with the type of the 2-primary component on the right side of (25).

If $d$ is even, the 2-primary components in (27) form the following exact sequence

$$
0 \longrightarrow \underbrace{\mathbb{Z}_{2^{k(p)-1}}^{\beta(G)-2} \oplus \mathbb{Z}_{2}}_{\text {type } \nu=\left((k(p)-1)^{\beta(G)-2}, 1\right)} \longrightarrow \underbrace{\operatorname{Syl}_{2} K}_{\text {type } \lambda} \longrightarrow \underbrace{\operatorname{Syl}_{2}(K(\operatorname{sd} G))}_{\text {type } \mu+\left(1^{\beta(G)}\right)} \stackrel{\pi}{\rightarrow} \mathbb{Z}_{2} \longrightarrow 0 .
$$

This can be truncated to the following short exact sequence involving $\operatorname{ker} \pi$ :

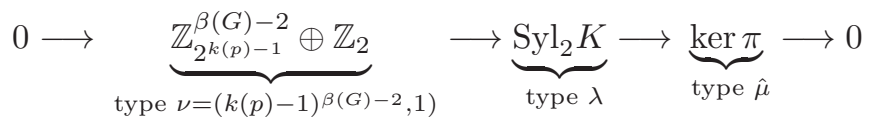

for some partition $\hat{\mu}$, and where the last two parts $\left(\lambda_{\beta(G)-1}, \lambda_{\beta(G)}\right)$ in $\lambda$ are either $(1,1)$ or $(2,0)$ by $(26)$, depending on the parity of $|V|$. 
The short exact sequence

$$
0 \rightarrow \operatorname{ker} \pi \rightarrow \operatorname{Syl}_{2}(K(\operatorname{sd} G)) \stackrel{\pi}{\rightarrow} \mathbb{Z}_{2} \rightarrow 0
$$

shows that $\hat{\mu}$ is obtained from $\mu+\left(1^{\beta(G)}\right)$ by removing one square; we claim that $\hat{\mu}$ can have at most $\beta(G)-1$ nonzero parts, and hence this square must be removed from the last row, that is, $\hat{\mu}=\mu+\left(1^{\beta(G)-1}, 0\right)$. The reason for this claim is that, since the LR-coefficient $c_{\hat{\mu}, \nu}^{\lambda} \neq 0$, the LR-condition forces

$$
\sum_{i \geq \beta(G)-1} \lambda_{i} \geq \sum_{i \geq \beta(G)-1} \hat{\mu}_{i}+\sum_{i \geq \beta(G)-1} \nu_{i}
$$

As $\sum_{i \geq \beta(G)-1} \lambda_{i}=2$ in both cases for the parity of $|V|$, and $\sum_{i \geq \beta(G)-1} \nu_{i}=1$, this forces $\sum_{i \geq \beta(G)-1} \hat{\mu}_{i} \leq 1$. This implies $\hat{\mu}$ can have at most $\beta(G)-1$ nonzero parts, as claimed.

Once one knows $\hat{\mu}$ takes this form, and since (26) fixes the shape of $\lambda$ in its last two rows $\beta(G)-1, \beta(G)$, any LR-tableau of shape $\lambda / \mu$ and content $\left.\nu=(k(p)-1)^{\beta(G)-2}, 1\right)$ is completely determined by column-strictness and the Yamanouchi condition: It must have its unique entry equal to $\beta(G)-1$ lying in the unique of cell of $\lambda / \mu$ within the last two rows, while all of its entries in row $i$ are all equal to $i$ for $i=1,2, \ldots, \beta(G)-2$. This again forces $\lambda_{i}=\mu_{i}+k(p)$ for $i=1,2, \ldots, \beta(G)-2$, and means that $\lambda$ again matches the type of the 2-primary component on the right side of (25).

Remark 7.1. In light of what Corollary 1.4 says about $K:=K$ (line $G$ ) for nonbipartite regular graphs, one might wonder what can be deduced for bipartite regular graphs using Theorems 1.2 and 1.3. We discuss this briefly here.

Fixing a prime $p$, define $k$ to be the largest exponent such that $p^{k}$ divides $d$, and let $\operatorname{Syl}_{p}(K(G))$ have type $\mu$. Then the $p$-primary components in the bipartite case of Theorem 1.3 form the following exact sequence:

$$
0 \rightarrow \underbrace{\mathbb{Z}_{p^{k}}^{\beta(G)-1}}_{\text {type } \nu=\left(k^{\beta(G)-1}\right)} \longrightarrow \underbrace{\operatorname{Syl}_{p} K}_{\text {type } \lambda} \longrightarrow \underbrace{\operatorname{Syl}_{p}(K(\operatorname{sd} G))}_{\begin{array}{c}
\text { type } \mu \\
\text { type } \mu+\left(1^{\beta(G)}\right) \quad \begin{array}{l}
\text { if } p \neq 2 \\
\text { if } p=2
\end{array}
\end{array}} \stackrel{\pi}{\longrightarrow} \underbrace{\mathbb{Z}_{p^{k}}}_{\text {type }(k)} \rightarrow 0
$$

As a consequence, $\operatorname{Syl}_{p}(K)$ will be uniquely determined by $\operatorname{Syl}_{p}(K(G))$ whenever $p$ does not divide $d$, since then $k=0$ and (31) shows $\operatorname{Syl}_{p}(K) \cong \operatorname{Syl}_{p}(K(\operatorname{sd} G))$ in this case. However, in general, the structures of $\operatorname{ker}(\pi)$ and of $\operatorname{Syl}_{p}(K)$ seem less clear. Even using the extra information from Theorem 1.2 that $K / p^{k(p)} K \cong \mathbb{Z}_{p^{k(p}}^{\beta(G)-1} \oplus \mathbb{Z}_{\operatorname{gcd}\left(p^{k},|V|\right)}$, where $k(p)$ is the largest power such that $p^{k(p)}$ divides $2 d$, along with the LR-rule, the structures of the various terms in the sequence are not uniquely determined.

Question 7.2. When $G$ is a simple, bipartite, regular graph, what more can be said about the structure of $K:=K($ line $G)$ in relation to that of $K(G)$ ?

\section{Proof of Theorem 1.5}

Let $G=(V, E)$ be a semiregular bipartite graph with vertex bipartition $V=V_{1} \sqcup V_{2}$, such that vertices in $V_{i}$ have degree $d_{i}$. In this section we prove our analogue of Theorem 1.3 for semiregular graphs. Recall that this is motivated by Cvetković's formula (3) for the spanning tree number of line $G$ :

$$
\kappa(\text { line } G)=\frac{\left(d_{1}+d_{2}\right)^{\beta(G)}}{d_{1} d_{2}}\left(\frac{d_{1}}{d_{2}}\right)^{\left|V_{2}\right|-\left|V_{1}\right|} \kappa(G) .
$$


We recall here the statement of Theorem 1.5.

Theorem 1.5. Let $G$ be a connected bipartite $\left(d_{1}, d_{2}\right)$-semiregular graph $G$. Then there is a group homomorphism

$$
K(\text { line } G) \stackrel{g}{\rightarrow} K(G)
$$

whose kernel-cokernel exact sequence

$$
0 \rightarrow \operatorname{ker}(g) \rightarrow K(\text { line } G) \stackrel{g}{\rightarrow} K(G) \rightarrow \operatorname{coker}(g) \rightarrow 0
$$

has

- $\operatorname{coker}(g)$ all $\operatorname{lcm}\left(d_{1}, d_{2}\right)$-torsion, and

- $\operatorname{ker}(g)$ all $\frac{d_{1}+d_{2}}{\operatorname{gcd}\left(d_{1}, d_{2}\right)} \operatorname{lcm}\left(d_{1}, d_{2}\right)$-torsion.

The proof of this result is analogous to that of Theorem 1.3; for this reason, some proofs here are either abbreviated or only sketched. Note also that this theorem is less precise than Theorem 1.3, partly out of necessity: Examples 8.6 and 8.7 below show that the morphism $g: K$ (line $G) \rightarrow K(G)$ appearing in the theorem is nearly surjective in some cases, but is the zero morphism in some other cases!

8.1. Defining the morphism $g$. We define $g$ similarly to the map $f$ from Definition 6.1. Let

$$
\begin{aligned}
\lambda & :=\operatorname{lcm}\left(d_{1}, d_{2}\right) \\
\gamma & :=\operatorname{gcd}\left(d_{1}, d_{2}\right) .
\end{aligned}
$$

As a notational convenience, denote typical vertices in $V_{1}$ (respectively, $V_{2}$ ) by $a$ 's (respectively, $b$ 's) with subscripts or primes.

Definition 8.1. For a semiregular bipartite graph $G$, let $g: \mathbb{R}^{E_{\text {line } G}} \rightarrow \mathbb{R}^{E_{G}}$ be defined $\mathbb{R}$-linearly by

$$
\begin{aligned}
& g\left(a b, b a^{\prime}\right)=\frac{\lambda}{d_{2}}\left((a, b)+\left(b, a^{\prime}\right)\right) \\
& g\left(b a, a b^{\prime}\right)=\frac{\lambda}{d_{1}}\left((b, a)+\left(a, b^{\prime}\right)\right) .
\end{aligned}
$$

Equivalently, the adjoint map $g^{t}$ is defined by

$$
g^{t}(a, b)=\frac{\lambda}{d_{1}} \sum_{b_{i} \in N(a)}\left(b_{i} a, a b\right)+\frac{\lambda}{d_{2}} \sum_{a_{j} \in N(b)}\left(a b, b a_{j}\right),
$$

where $N(v)$ denotes the set of vertices adjacent to $v$ in $G$.

Remark 8.2. In the special case when $G$ is not only semiregular bipartite, but actually regular, so $d_{1}=d_{2}=\lambda=\gamma$, one can easily check that the map $g$ coincides with the composite map $h \circ f$

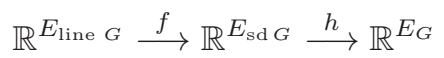

where $f$ is the map from Theorem 1.3 defined in Definition 6.1, and $h$ was defined in Example 3.3.

Proposition 8.3. If $G$ is a semiregular bipartite graph, then $g: \mathbb{Z}^{E_{\text {line }}} \rightarrow \mathbb{Z}^{E_{G}}$ is a morphism of the associated rational orthgonal decompositions, and hence induces a group homomorphism $g: K($ line $G) \rightarrow K(G)$. 
Proof. By Lemma 6.3 (ii), it is enough to show that $g$ takes global and local cycles in $Z_{\text {line } G}$ to cycles in $Z_{G}$, and that $g^{t}$ takes cycles in $Z_{G}$ to cycles in $Z_{\text {line } G}$.

First, one can check that $g$ maps all local cyles to 0 . Each global cycle is by definition of the form $z$ (line $C$ ) where $C$ is a directed cycle of $G$, and one checks that

$$
g(z(\text { line } C))=\left(\frac{\lambda}{d_{1}}+\frac{\lambda}{d_{2}}\right) z(C) .
$$

On the other hand, one checks that $g^{t}(z(C))$ can be rewritten as a sum of $\lambda$ cycles $\zeta_{i}$ in $Z_{\text {line } G}$, each $\zeta_{i}$ being twice the length of $C$, and in which every other vertex on $\zeta_{i}$ corresponds to an edge occurring in $C$.

\subsection{Analyzing its kernel and cokernel.}

Proposition 8.4. The map

$$
g^{t} g: K(\text { line } G) \rightarrow K(\text { line } G)
$$

coincides with scalar multiplication by $\frac{d_{1}+d_{2}}{\gamma} \lambda$. Consequently, $\operatorname{ker}(g)$ is $\frac{d_{1}+d_{2}}{\gamma} \lambda$-torsion.

Proof. For any edge $a b, b a^{\prime}$ in $E_{\text {line } G}$, use the definitions of $g$ and $g^{t}$ to write

$$
\begin{aligned}
g^{t} g\left(a b, b a^{\prime}\right)= & \frac{\lambda^{2}}{d_{1} d_{2}} \sum_{b_{i} \in N(a)}\left(b_{i} a, a b\right)+\frac{\lambda^{2}}{d_{2}^{2}} \sum_{a_{j} \in N(b)}\left(a b, b a_{j}\right) \\
& +\frac{\lambda^{2}}{d_{2}^{2}} \sum_{a_{j} \in N(b)}\left(a_{j} b, b a^{\prime}\right)+\frac{\lambda^{2}}{d_{1} d_{2}} \sum_{b_{k} \in N\left(a^{\prime}\right)}\left(b a^{\prime}, a^{\prime} b_{k}\right) .
\end{aligned}
$$

For the first and fourth term, one has

$$
\begin{aligned}
\sum_{b_{i} \in N(a)}\left(b_{i} a, a b\right) & =\sum_{a_{j} \in N(b)}\left(a b, b a_{j}\right) \bmod B_{\text {line } G} \\
\sum_{b_{k} \in N\left(a^{\prime}\right)}\left(b a^{\prime}, a^{\prime} b_{k}\right) & =\sum_{a_{j} \in N(b)}\left(a_{j} b, b a^{\prime}\right) \bmod B_{\text {line } G .} .
\end{aligned}
$$

Substituting these expressions into equation (33), grouping like terms, and using the identity $d_{1} d_{2}=\lambda \gamma$ gives

$$
g^{t} g\left(a b, b a^{\prime}\right)=\frac{d_{1}+d_{2}}{\gamma} \cdot \frac{\lambda}{d_{2}}\left(\sum_{a_{j} \in N(b)}\left(a b, b a_{j}\right)+\sum_{a_{j} \in N(b)}\left(a_{j} b, b a^{\prime}\right)\right) \bmod B_{\text {line } G},
$$

which then can be rewritten, using the $d_{2}$ triangular cycles

$$
\left(a b, b a_{j}\right)+\left(a_{j} b, b a^{\prime}\right)+\left(a^{\prime} b, b a\right) \in Z_{\text {line } G},
$$

as

$$
\begin{aligned}
g^{t} g\left(a b, b a^{\prime}\right) & =\frac{\left(d_{1}+d_{2}\right)}{\gamma} \cdot \frac{\lambda}{d_{2}}\left(d_{2}\left(a b, b a^{\prime}\right)\right) \bmod B_{\text {line } G}+Z_{\text {line } G} \\
& =\frac{\left(d_{1}+d_{2}\right)}{\gamma} \lambda\left(a b, b a^{\prime}\right) \bmod B_{\text {line } G}+Z_{\text {line } G} \cdot
\end{aligned}
$$

Remark 8.5. As in Proposition 6.5, one can show that the other map $g g^{t}: K(G) \rightarrow K(G)$ also coincides with the scalar multiplication by $\frac{d_{1}+d_{2}}{\gamma} \lambda$, and hence that $\operatorname{coker}(g)$ is also $\frac{d_{1}+d_{2}}{\gamma} \lambda$-torsion. However, we omit this proof, since we are about to show the stronger assertion that $\operatorname{coker}(g)$ is $\lambda$-torsion. 
Proof of Theorem 1.5. In light of Proposition 8.4, it only remains to show that $\operatorname{coker}(g)$ is $\lambda$-torsion. Given any edge $a b \in E_{G}$, one has

$$
\begin{aligned}
\lambda(a, b) & =\lambda(a, b)+\frac{\lambda}{d_{2}} \sum_{a_{j} \in N(b)}\left(b, a_{j}\right) \bmod B_{G} \\
& =\frac{\lambda}{d_{2}} \sum_{a_{j} \in N(b)}(a, b)+\left(b, a_{j}\right) \bmod B_{G} \\
& =g\left(\sum_{a_{j} \in N(b)}\left(a b, b a_{j}\right)\right) \bmod B_{G} .
\end{aligned}
$$

Consequently $\lambda(a, b)$ lies in $\operatorname{im}(g)+B_{G}$, so it is zero in $\operatorname{coker}(g):=\mathbb{Z}^{E_{G}} /\left(\operatorname{im}(g)+B_{G}+Z_{G}\right)$.

Unlike the map $f$ from Section 6 , it is hard to be much more precise about the exact nature of cokernel and kernel of $g$. The following two families of examples demonstrate two extremes of behavior for how tightly or loosely the map $g$ ties together $K$ (line $G$ ) and $K(G)$ for semiregular bipartite graphs $G$.

Example 8.6. Assume $G$ is not only bipartite semiregular, but actually $d$-regular (i.e., $d_{1}=d_{2}=d$ ). Then $g: K$ (line $\left.G\right) \rightarrow K(G)$ is nearly surjective, in the sense that coker $(g)$ is a quotient of $\mathbb{Z}_{d}$. To see this, recall from Remark 8.2 that in this case, $g=h \circ f$ where $h, f$ were defined in Example 3.3 and Definition 6.1. Since $h: K(\operatorname{sd} G) \rightarrow K(G)$ is surjective, it induces a surjection

$$
\operatorname{coker}(f):=K(\operatorname{sd} G) / \operatorname{im}(f) \longrightarrow K(G) / \operatorname{im}(h \circ f)=: \operatorname{coker}(g) .
$$

But Theorem 1.3 says that $\operatorname{coker}(f)=\mathbb{Z}_{d}$ in this situation.

Example 8.7. For the complete bipartite graph $G=K_{n_{1}, n_{2}}$, the structures of the critical groups of $G$ and line $G$ have been determined through manipulations of their Laplacian matrices (see Lorenzini [13] and Berget [3], respectively):

$$
\begin{aligned}
K\left(K_{n_{1}, n_{2}}\right) & \cong \mathbb{Z}_{n_{1}}^{n_{2}-2} \oplus \mathbb{Z}_{n_{2}}^{n_{1}-2} \oplus \mathbb{Z}_{n_{1} n_{2}}, \\
K\left(\text { line } K_{n_{1}, n_{2}}\right) & \cong \mathbb{Z}_{n_{1}\left(n_{1}+n_{2}\right)}^{n_{1}-2} \oplus \mathbb{Z}_{n_{2}\left(n_{1}+n_{2}\right)}^{n_{2}-2} \oplus \mathbb{Z}_{n_{1}+n_{2}}^{\left(n_{1}-2\right)\left(n_{2}-2\right)+1} .
\end{aligned}
$$

In principle, the structures of these groups allow nonzero homomorphisms between them for all values of $n_{1}, n_{2}$. However, we claim that whenever $\gamma=\operatorname{gcd}\left(n_{1}, n_{2}\right)=1$, the map $K($ line $G) \stackrel{g}{\rightarrow} K(G)$ will be the zero morphism. In this case, $\operatorname{ker}(g)=K($ line $G$ ) and $\operatorname{coker}(g)=K(G)$.

To see this claim, let $\left(a b, b a^{\prime}\right)$ be a fixed edge in $E_{\text {line }} G$. Note that

$$
\begin{aligned}
\lambda & =d_{1} d_{2}=n_{1} n_{2}, \\
d_{1} & =n_{2}, d_{2}=n_{1} .
\end{aligned}
$$

Then for each $b_{j} \in V_{2}$, one has

$$
\frac{1}{d_{1}}\left(g\left(a b, b a^{\prime}\right)+g\left(a^{\prime} b_{j}, b_{j} a\right)\right)=a b+b a^{\prime}+a^{\prime} b_{j}+b_{j} a \in Z_{G} .
$$


On the other hand,

$$
\begin{aligned}
\sum_{b_{j} \in V_{2}} \frac{1}{d_{1}}\left(g\left(a b, b a^{\prime}\right)+g\left(a^{\prime} b_{j}, b_{j} a\right)\right) & =g\left(a b, b a^{\prime}\right)+\sum_{b_{j} \in V_{2}} \frac{1}{d_{1}} \frac{\lambda}{d_{2}}\left(a^{\prime} b_{j}+b_{j} a\right) \\
& =g\left(a b, b a^{\prime}\right)+\sum_{b_{j} \in V_{2}} a^{\prime} b_{j}+\sum_{b_{j} \in V_{2}} b_{j} a \\
& =g\left(a b, b a^{\prime}\right) \bmod B_{G} .
\end{aligned}
$$

Combining these two statements gives us $g\left(a b, b a^{\prime}\right)=0 \bmod Z_{G}+B_{G}$. By symmetry, one also has $g\left(b a, a b^{\prime}\right)=0 \bmod Z_{G}+B_{G}$. It follows that $g$ is the zero morphism.

Remark 8.8. Note that Theorem 1.2 provides convenient information about $\operatorname{Syl}_{p}(K)$ for $K:=K($ line $G)$ when $G$ is $\left(d_{1}, d_{2}\right)$-semiregular: If $k(p)$ denotes the largest power $p^{k(p)}$ dividing $d_{1}+d_{2}$, then

$$
K / p^{k(p)} K \cong \mathbb{Z}_{p^{k(p)}}^{\beta(G)-1} \oplus \mathbb{Z}_{\operatorname{gcd}\left(p^{k(p)},|V|\right)} .
$$

However, even in conjunction with Theorem 1.5, this does not appear to determine the structure of $K$ (line $G$ ) uniquely in terms of the structure of $K(G)$. Thus we are led to the following generalization of Question 7.2:

Question 8.9. When $G$ is a simple, semiregular bipartite graph, what more can be said about the structure of $K:=K($ line $G)$ in relation to that of $K(G)$ ?

\section{ExAmples}

9.1. The complete graph $K_{n}$. Proposition 3.3 can be rephrased as asserting that

$$
K\left(K_{n}\right) \cong \mathbb{Z}_{n}^{n-2} \oplus \mathbb{Z}_{1}^{\beta-n+2}
$$

where $\beta:=\beta\left(K_{n}\right)=\left(\begin{array}{c}n-1 \\ 2\end{array}\right)$. Since $K_{n}$ is nonbipartite for $n \geq 3$, and contains an even length cycle for $n \geq 4$, Corollary 1.4 immediately implies the following:

Corollary 9.1. For $n \geq 4$, the line graph line $K_{n}$ of the critical group of the complete graph $K_{n}$ has the form

$$
\begin{aligned}
& K\left(\text { line } K_{4}\right)=\mathbb{Z}_{24} \oplus \mathbb{Z}_{8} \oplus \mathbb{Z}_{2} \\
& K\left(\text { line } K_{n}\right)=\mathbb{Z}_{2(n-1) n}^{n-2} \oplus \mathbb{Z}_{2(n-1)}^{\beta-n} \oplus \begin{cases}\mathbb{Z}_{2}^{2} & \text { for even } n>5, \\
\mathbb{Z}_{4} & \text { for odd } n \geq 5 .\end{cases}
\end{aligned}
$$

9.2. The complete bipartite graph $K_{n_{1}, n_{2}}$. As mentioned in Example 8.7, the critical groups of the complete bipartite graph $K_{n_{1}, n_{2}}$ and its line graph line $K_{n_{1}, n_{2}}$ have the following forms:

$$
\begin{aligned}
K\left(K_{n_{1}, n_{2}}\right) & \cong \mathbb{Z}_{n_{1}}^{n_{2}-2} \oplus \mathbb{Z}_{n_{2}}^{n_{1}-2} \oplus \mathbb{Z}_{n_{1} n_{2}} \\
K\left(\text { line } K_{n_{1}, n_{2}}\right) & \cong \mathbb{Z}_{n_{1}\left(n_{1}+n_{2}\right)}^{n_{1}-2} \oplus \mathbb{Z}_{n_{2}\left(n_{1}+n_{2}\right)}^{n_{2}-2} \oplus \mathbb{Z}_{n_{1}+n_{2}}^{\left(n_{1}-2\right)\left(n_{2}-2\right)+1}
\end{aligned}
$$

(see Lorenzini [13] and Berget [3], respectively).

In addition, Example 8.7 showed that the map $g$ in the exact sequence in Theorem 1.5 is sometimes the zero morphism and hence is not always useful for determining the structure of 
$K$ (line $K_{n_{1}, n_{2}}$ ). Even in the special case when $n_{1}=n_{2}=n$ (so $K$ (line $\left.K_{n, n}\right)$ is $n$-regular), the exact sequence

$$
0 \rightarrow \underbrace{\mathbb{Z}_{n}^{\beta-2} \oplus \mathbb{Z}_{n}}_{\mathbb{Z}_{n}^{n(n-2)}} \longrightarrow \underbrace{K\left(\text { line } K_{n, n}\right)}_{\mathbb{Z}_{2 n^{2}}^{2(n-2)} \oplus \mathbb{Z}_{2 n}^{(n-2)^{2}+1}} \stackrel{f}{\longrightarrow} \underbrace{K\left(\operatorname{sd} K_{n, n}\right)}_{\mathbb{Z}_{2 n^{2}}^{1} \oplus \mathbb{Z}_{2 n}^{2(n-2)} \oplus \mathbb{Z}_{2}^{(n-2)^{2}}} \longrightarrow \mathbb{Z}_{n} \rightarrow 0
$$

from Theorem 1.3 does not determine a priori Berget's formula for $K\left(\right.$ line $K_{n, n}$ ).

However, we note that at least Theorem 1.2 does predict that the expression

$$
K\left(\text { line } K_{n_{1}, n_{2}}\right)=\bigoplus_{i=1}^{\beta} \mathbb{Z}_{e_{i}} \text { where } \beta:=\beta\left(K_{n_{1}, n_{2}}\right)=\left(n_{1}-1\right)\left(n_{2}-1\right)
$$

should have $|V|=n_{1}+n_{2}$ dividing every one of the factors $e_{i}$. This follows from equation (34), since $K_{n_{1}, n_{2}}$ is bipartite $\left(n_{1}, n_{2}\right)$-semiregular. Hence for each prime $p$, one has

$$
K / p^{k(p)} K \cong \mathbb{Z}_{p^{k(p)}}^{\beta-1} \oplus \mathbb{Z}_{\operatorname{gcd}\left(p^{k(p)},|V|\right)}=\mathbb{Z}_{p^{k(p)}}^{\beta},
$$

where $k(p)$ is the largest power such that $p^{k(p)}$ divides $n_{1}+n_{2}$. Hence $K /\left(n_{1}+n_{2}\right) K \cong$ $\mathbb{Z}_{n_{1}+n_{2}}^{\beta}$.

9.3. The $d$-dimensional cube. Denote by $G_{\mathrm{d} \text {-cube }}$ the graph of vertices and edges in the $d$-dimensional cube, that is, $G_{\mathrm{d} \text {-cube }}=(V, E)$ in which $V$ is the set of all binary strings of length $d$, and $E$ has an edge between any two such strings that differ in exactly one binary digit. This is a $d$-regular bipartite graph, having

$$
\beta:=\beta\left(G_{\mathrm{d} \text {-cube }}\right)=(d-2) 2^{d-1}+1 .
$$

One knows its spanning tree number (see, e.g., [17, Example 5.6.10]):

$$
\kappa\left(G_{\text {d-cube }}\right)=\frac{1}{2^{d}} \prod_{k=1}^{d}(2 k)^{\left(\begin{array}{l}
d \\
k
\end{array}\right)}=2^{2^{d}-d-1} \prod_{k=2}^{d} k^{\left(\begin{array}{l}
d \\
k
\end{array}\right)} .
$$

Correspondingly, work of $\mathrm{H}$. Bai [2] computes its critical group structure away from the prime 2: For odd primes $p$, one has

$$
\operatorname{Syl}_{p}\left(K\left(G_{\text {d-cube }}\right)\right)=\operatorname{Syl}_{p}\left(\bigoplus_{k=2}^{d} \mathbb{Z}_{k}^{\left(\begin{array}{l}
d \\
k
\end{array}\right)}\right) .
$$

Unfortunately, $\operatorname{Syl}_{2}\left(K\left(G_{\mathrm{d} \text {-cube }}\right)\right)$ is a 2 -group that is still not known for all $d$.

Consequently, Proposition 3.2 shows that $K\left(\operatorname{sd}\left(G_{\text {d-cube }}\right)\right)$ has the same $p$-primary structure as $K\left(G_{\text {d-cube }}\right)$ for odd primes $p$, and Theorem 1.3 gives the following exact sequence for every odd prime $p$ :

$$
0 \rightarrow \operatorname{Syl}_{p}\left(\mathbb{Z}_{d}^{\beta-1}\right) \rightarrow \operatorname{Syl}_{p}\left(K\left(\text { line } G_{\text {d-cube }}\right)\right) \rightarrow \operatorname{Syl}_{p}\left(\bigoplus_{k=2}^{d} \mathbb{Z}_{k}^{\left(\begin{array}{l}
d \\
k
\end{array}\right)}\right) \rightarrow \mathbb{Z}_{d} \rightarrow 0 .
$$

This is particularly effective when $d$ itself is prime since then $\operatorname{Syl}_{d}\left(\bigoplus_{k=2}^{d} \mathbb{Z}_{k}^{\left(\begin{array}{l}d \\ k\end{array}\right)}\right)=\mathbb{Z}_{d}$ and the exact sequence (35) implies that for odd primes $p$,

$$
\operatorname{Syl}_{p}\left(K\left(\text { line } G_{\text {d-cube }}\right)\right)= \begin{cases}\mathbb{Z}_{d}^{\beta-1} & \text { if } p=d \\
\operatorname{Syl}_{p}\left(\bigoplus_{k=2}^{d-1} \mathbb{Z}_{k}^{\left(\begin{array}{l}
d \\
k
\end{array}\right)}\right) & \text { if } p \neq d .\end{cases}
$$


Meanwhile $\operatorname{Syl}_{2}\left(K\left(\right.\right.$ line $\left.\left.G_{\text {d-cube }}\right)\right)=\operatorname{Syl}_{2}\left(K\left(\operatorname{sd}\left(G_{\text {d-cube }}\right)\right)\right)$ is unknown, but by Proposition 3.2, is completely determined by the unknown 2-group $\operatorname{Syl}_{2}\left(K\left(G_{\mathrm{d} \text {-cube }}\right)\right)$.

9.4. The Platonic solids. One source of regular graphs are the 1-skeleta (= graph of vertices and edges) of the Platonic solids. There are certain features that apply to any graph $G_{P}$ which is the 1-skeleton of a 3-dimensional polyhedron $P$, and hence to any Platonic solid:

- Because the cycles surrounding the (polygonal) faces of $P$ generate the cycle lattice $Z$, the graph $G_{P}$ is bipartite if and only if each face of $P$ is an even $n$-gon.

- Furthermore, the cycles that bound all but one face of $P$ form a basis for $Z$, so that $\beta\left(G_{P}\right)$ is always one less than the number of faces.

- Such graphs $G_{P}$ are always 2-edge-connected, so that Theorem 1.1 always applies.

- Dual polyhedra $P, P^{*}$ have $G_{P}, G_{P^{*}}$ dual as planar graphs. This identifies the lattice of bonds for one with the lattice of cycles for the other, and implies that their critical groups $K\left(G_{P}\right), K\left(G_{P *}\right)$ are isomorphic; see also [6].

9.4.1. The tetrahedron. The tetrahedron has 1-skeleton $G_{\text {tetra }}=K_{4}$, and hence implicitly was discussed already in Section 9.1 on $K_{n}$, as the special case $n=4$.

9.4.2. The cube and octahedron. The cube and the octahedron are dual polyhedra. Either by direct computer calculation, or by noting $G_{\text {octa }} \cong$ line $K_{4}$ and applying Corollary 9.1 with $n=4$, one finds that

$$
\begin{aligned}
K\left(G_{\text {cube }}\right)=K\left(G_{\text {octa }}\right) & =\mathbb{Z}_{2} \oplus \mathbb{Z}_{8} \oplus \mathbb{Z}_{24} \\
& =\mathbb{Z}_{2} \oplus \mathbb{Z}_{8}^{2} \oplus \mathbb{Z}_{3} .
\end{aligned}
$$

Since $G_{\text {octa }}$ is 4-regular and nonbipartite with $\beta\left(G_{\text {octa }}\right)=7$, Corollary 1.4 then implies

$$
K\left(\text { line } G_{\text {octa }}\right)=\mathbb{Z}_{2}^{2} \oplus \mathbb{Z}_{8}^{2} \oplus \mathbb{Z}_{16} \oplus \mathbb{Z}_{64} \oplus \mathbb{Z}_{192} \text {. }
$$

For $G_{\text {cube }}$, which has $\beta\left(G_{\text {cube }}\right)=5$, the results of Section 9.3 apply, and are particularly effective because $d=3$ is prime. They show that $\operatorname{Syl}_{p}\left(K\left(\right.\right.$ line $\left.\left.G_{\text {d-cube }}\right)\right)$ vanishes except for $p=2,3$, with

$$
\begin{aligned}
& \operatorname{Syl}_{3}\left(K\left(\text { line } G_{\text {d-cube }}\right)\right)=\mathbb{Z}_{3}^{4} \\
& \operatorname{Syl}_{2}\left(K\left(\text { line } G_{\text {d-cube }}\right)\right)=\operatorname{Syl}_{2}\left(K\left(\operatorname{sd}\left(G_{\text {d-cube }}\right)\right)\right)=\mathbb{Z}_{2}^{2} \oplus \mathbb{Z}_{4} \oplus \mathbb{Z}_{16}^{2} .
\end{aligned}
$$

Hence

$$
\begin{aligned}
K\left(\text { line } G_{\text {cube }}\right) & =\mathbb{Z}_{2}^{2} \oplus \mathbb{Z}_{4} \oplus \mathbb{Z}_{16}^{2} \oplus \mathbb{Z}_{3}^{4} \\
& =\mathbb{Z}_{2} \oplus \mathbb{Z}_{6} \oplus \mathbb{Z}_{12} \oplus \mathbb{Z}_{48}^{2} .
\end{aligned}
$$

9.4.3. The dodecahedron and icosahedron. The dodecahedron and icosahedron are dual polyhedra, both of whose graphs are nonbipartite. Computer calculation shows that

$$
\begin{aligned}
K\left(G_{\text {dodeca }}\right)=K\left(G_{\text {icosa }}\right) & =\mathbb{Z}_{2} \oplus \mathbb{Z}_{12} \oplus \mathbb{Z}_{60}^{3} \\
& =\mathbb{Z}_{2} \oplus \mathbb{Z}_{4}^{4} \oplus \mathbb{Z}_{3}^{4} \oplus \mathbb{Z}_{5}^{3} .
\end{aligned}
$$

Since $G_{\text {dodeca }}$ is 5 -regular with $\beta\left(G_{\text {dodeca }}\right)=11$, one concludes from Corollary 1.4 that

$$
\begin{aligned}
K\left(\text { line } G_{\text {dodeca }}\right) & =\mathbb{Z}_{2}^{2} \oplus \mathbb{Z}_{6}^{4} \oplus \mathbb{Z}_{12} \oplus \mathbb{Z}_{72} \oplus \mathbb{Z}_{360}^{3} \\
& =\mathbb{Z}_{2}^{6} \oplus \mathbb{Z}_{4} \oplus \mathbb{Z}_{8}^{4} \oplus \mathbb{Z}_{3}^{5} \oplus \mathbb{Z}_{9}^{4} \oplus \mathbb{Z}_{5}^{3} .
\end{aligned}
$$

Since $G_{\text {icosa }}$ is 3 -regular with $\beta\left(G_{\text {icosa }}\right)=19$, one concludes from Corollary 1.4 that

$$
\begin{aligned}
K\left(\text { line } G_{\text {icosa }}\right) & =\mathbb{Z}_{2}^{2} \oplus \mathbb{Z}_{10}^{12} \oplus \mathbb{Z}_{20} \oplus \mathbb{Z}_{120} \oplus \mathbb{Z}_{600}^{3} \\
& =\mathbb{Z}_{2}^{14} \oplus \mathbb{Z}_{4} \oplus \mathbb{Z}_{8}^{4} \oplus \mathbb{Z}_{3}^{4} \oplus \mathbb{Z}_{5}^{14} \oplus \mathbb{Z}_{25}^{3} .
\end{aligned}
$$




\section{ACKNOWLEDGMENTS}

The authors thank the anonymous referee for a careful reading of the manuscript and many helpful suggestions, and also thank David Treumann for allowing them to include some of his results here.

\section{REFERENCES}

[1] R. Bacher, P. de la Harpe, and T. Nagnibeda, The lattice of integral cuts and lattice of integral flows of a finite graph. Bull. Soc. Math. France 125 (1997), 167-198.

[2] H. Bai, On the critical group of the n-cube, Lin. Algebra Appl. 369 (2003), 251-261.

[3] A. Berget, Critical groups of some regular line graphs, (2003). Available online at http://www . math.ucdavis . edu/ berget/research/

[4] N. Biggs, Algebraic potential theory on graphs, Bull. London Math. Soc. 29 (1997), 641-682.

[5] N. Biggs, Algebraic Graph Theory. Cambridge University Press, 1993.

[6] R. Cori and D. Rossin, On the sandpile group of dual graphs. European J. Combin. 21 (2000), no. 4, $447-459$.

[7] D. Cvetković, M. Doob, and H. Sachs, Spectra of graphs. Theory and application. Pure and Applied Mathematics 87. Academic Press, Inc. [Harcourt Brace Jovanovich, Publishers], New York-London, 1980.

[8] C. Godsil and G. Royle, Algebraic Graph Theory, Springer-Verlag, 2001.

[9] N. Hartsfield and G. Ringel, Pearls in Graph Theory: A Comprehensive Introduction. Academic Press, 1994.

[10] B. Jacobson, A. Neidermeier and V. Reiner, Critical groups for complete multipartite graphs and Cartesian products of complete graphs. J. Graph Theory 44 (2003), 231-250

[11] A. K. Kelmans, On properties of the characteristic polynomial of a graph, Kibernetiku-Na Sluzbu Kom., Gosenergoizdat, Moscow, 4 (1967), 27-47 (in Russian).

[12] L. Levine, Sandpile groups and spannings trees of directed line graphs. Preprint (2009), to appear in J. Comb. Theory Ser. A.

[13] D. Lorenzini, A finite group attached to the laplacian of a graph. Discrete Mathematics 91 (1991), $277-282$.

[14] I.G. Macdonald, Symmetric functions and Hall polynomials, 2nd edition. Oxford Mathematical Monographs. Oxford Science Publications. The Clarendon Press, Oxford University Press, New York, 1995.

[15] B. Mohar, The Laplacian Spectrum of Graphs. Graph Theory, Combinatorics, and Applications 2 Ed. by Y. Alavi, G. Chartrand, O. R. Oellermann, A. J. Schwenk. Wiley, 1991, 871-898.

[16] M. Rubey, Counting Spanning Trees. Diplomarbeit, Universität Wien, 2000.

[17] R.P. Stanley, Enumerative Combinatorics, Vol. 2. Cambridge Studies in Advanced Mathematics 62. Cambridge University Press, Cambridge, 1999.

[18] R.P. Stanley, A zonotope associated with graphical degree sequences. Applied geometry and discrete mathematics, 555-570, DIMACS Ser. Discrete Math. Theoret. Comput. Sci. 4, Amer. Math. Soc., Providence, RI, 1991.

[19] D. Treumann, Functoriality of critical groups, Bachelor's thesis, Univ. of Minnesota, 2002, Available online at http://www.math.umn.edu/ reiner/REU/REU.html.

[20] E.B. Vahovskii, On the characteristic numbers of incidence matrices for non-singular graphs, Sibirsk. Mat. Zh. 6 (1965) 44-49 (in Russian).

[21] D. West, Introduction to Graph Theory. Prentice Hall, 2001.

E-mail address: berget@math.ucdavis.edu

E-mail address: andymanion@gmail.com

E-mail address: molly.maxwell@coloradocollege.edu

E-mail address: potechin@mit.edu

E-mail address: reiner@math.umn.edu

Department of Mathematics, University of California, Davis, CA 95616

Department of Mathematics, Princeton University, Princeton, NJ 08540 
Department of Mathematics and Computer Science, Colorado College, 14 E. Cache la Poudre St., Colorado Springs, CO 80903

Department of Mathematics, Massachusetts institute of Technology, Cambridge, Ma 02139

School of Mathematics, University of Minnesota, 206 Church St. S.E., Minneapolis, Mn 55455 